\title{
Future Missions Related to the Determination of the Elemental and Isotopic Composition of Earth, Moon and the Terrestrial Planets
}

\author{
Iannis Dandouras ${ }^{1}$ D $\cdot$ Michel Blanc ${ }^{1} \cdot$ Luca Fossati $^{2} \cdot$ Mikhail Gerasimov $^{3}$. \\ Eike W. Guenther ${ }^{4}$ - Kristina G. Kislyakova ${ }^{2,5}$ • Helmut Lammer ${ }^{2}$. Yangting Lin ${ }^{6}$. \\ Bernard Marty $^{7}$. Christian Mazelle ${ }^{1} \cdot$ Sarah Rugheimer $^{8} \cdot$ Manuel Scherf $^{2}$. \\ Christophe Sotin $^{9}$ Laurenz Sproß ${ }^{2,10}$ - Shogo Tachibana ${ }^{11}$ - Peter Wurz ${ }^{12}$. \\ Masatoshi Yamauchi ${ }^{13}$
}

Received: 8 January 2020 / Accepted: 25 September 2020 / Published online: 22 October 2020

(C) The Author(s) 2020

\begin{abstract}
In this chapter, we review the contribution of space missions to the determination of the elemental and isotopic composition of Earth, Moon and the terrestrial planets, with special emphasis on currently planned and future missions. We show how these missions are going to significantly contribute to, or sometimes revolutionise, our understanding of planetary evolution, from formation to the possible emergence of life. We start with the Earth, which is a unique habitable body with actual life, and that is strongly related to its atmo-
\end{abstract}

Reading Terrestrial Planet Evolution in Isotopes and Element Measurements

Edited by Helmut Lammer, Bernard Marty, Aubrey L. Zerkle, Michel Blanc, Hugh O'Neill and Thorsten Kleine

\section{Dandouras}

Iannis.Dandouras@irap.omp.eu

1 Institut de Recherche en Astrophysique et Planétologie, Université de Toulouse / CNRS / UPS /

CNES, Toulouse, France

2 Space Research Institute, Austrian Academy of Sciences, Graz, Austria

3 Space Research Institute of RAS (IKI), Moscow, Russia

4 Thüringer Landessternwarte Tautenburg, Tautenburg, Germany

5 Department of Astrophysics, University of Vienna, Vienna, Austria

6 Institute of Geology and Geophysics, Chinese Academy of Sciences, Beijing, China

7 Nancy Université, Nancy, France

8 Department of Physics, Oxford University, Oxford, UK

9 Jet Propulsion Laboratory, NASA, Pasadena, CA, USA

10 Institute of Physics, University of Graz, Graz, Austria

11 Hokkaido University, Sapporo, Japan

12 Physikalisches Institut, University of Bern, Bern, Switzerland

13 Swedish Institute of Space Physics (IRF), Kiruna, Sweden 
sphere. The new wave of missions to the Moon is then reviewed, which are going to study its formation history, the structure and dynamics of its tenuous exosphere and the interaction of the Moon's surface and exosphere with the different sources of plasma and radiation of its environment, including the solar wind and the escaping Earth's upper atmosphere. Missions to study the noble gas atmospheres of the terrestrial planets, Venus and Mars, are then examined. These missions are expected to trace the evolutionary paths of these two noble gas atmospheres, with a special emphasis on understanding the effect of atmospheric escape on the fate of water. Future missions to these planets will be key to help us establishing a comparative view of the evolution of climates and habitability at Earth, Venus and Mars, one of the most important and challenging open questions of planetary science. Finally, as the detection and characterisation of exoplanets is currently revolutionising the scope of planetary science, we review the missions aiming to characterise the internal structure and the atmospheres of these exoplanets.

Keywords Space missions · Planetary evolution · Planetary atmospheres · Planetary magnetospheres $\cdot$ Terrestrial planets $\cdot$ Exoplanets $\cdot$ Biosignatures

\section{Introduction}

The previous chapters of this topical series presented in considerable detail what we know on the way the evolution of a planet and its atmosphere, from formation to the possible emergence of habitable worlds, influences its elemental, chemical and isotopic composition. Indeed, the chemical and isotopic composition of the different constituents of a planet are shaped by initial conditions such as the composition and activity of its host star (Güdel 2020) and the structure and composition of the circumstellar nebula out of which it was born (Bermingham et al. 2020). In the course of its evolution, each planetary atmosphere is fed by additional material from its space environment and from the planet's degassing interior (Stüeken et al. 2020) while a fraction of its atmospheric chemical elements escape to outer space (Lammer et al. 2020a). A quantitative assessment of the effects of these mechanisms can be partly constrained in the chemical and isotopic composition of the different planetary layers, among them its atmosphere, and in the meteorite record (e.g., O'Neill et al. 2020) where they can be most easily measured. These measurements provide constraints on the formation history of Earth, Moon and the terrestrial planets (Lammer et al. 2020b), on the evolution of planetary atmospheres (Avice and Marty 2020; Stüeken et al. 2020) and on their current composition (Scherf et al. 2020). Last but not least, when life emerges, like on Earth, it also leaves a strong imprint on the planet's composition (Lloyd et al. 2020).

In this article, we review the contribution of space missions to Earth, Moon and the terrestrial planets, to the determination of their elemental and isotopic composition, with special emphasis on currently planned and future missions. We show how these missions are going to strongly contribute to, or sometimes revolutionise, our understanding of planetary evolution, from formation to the possible emergence of life. Our scope is not to provide an exhaustive list of missions, but to highlight some missions to the inner planets of our Solar System addressing these objectives, and then to expand our review to missions aiming to characterise planets around other stars.

We start this review with missions to the Earth-Moon system (Sect. 2). Building on the legacy of Apollo and of several other important missions to the Moon, we review the current plans for a new wave of Moon missions from a broad range of countries and from the public and private spheres. These missions, combining orbiter and lander elements, are aimed at 
studying the formation history of the Moon, the structure and dynamics of its tenuous exosphere, and the interaction of the Moon's surface and exosphere with the different sources of plasma and radiation of its environment, including the solar wind and the escaping Earth's upper atmosphere. We also review the current plans for missions in Earth orbit that will help better quantify the atmospheric losses processes and also their effects on the Earth's upper atmosphere and magnetosphere.

In Sect. 3 we review past studies and current plans to use noble gases in the atmospheres of the terrestrial planets, Venus and Mars, to trace the evolutionary paths of these atmospheres, with a special emphasis on understanding the effect of atmospheric escape on the fate of water. Future missions to these planets will be key to help us establish a comparative view of the evolution of climates and habitability at Earth Venus and Mars, one of the most important and challenging open questions of planetary science. In a similar way, past (Messenger) and current (BepiColombo) missions to Mercury are expected to provide a new view of the bulk and exosphere composition of this planet, the only example of a hot close-in terrestrial planet in our Solar System.

While remote sensing and in situ measurements are expected to provide new insights into these questions, return of samples of surface, sub-surface and atmospheres to our Earth laboratories is the most promising perspective to write an accurate evolutionary history of terrestrial planets, as illustrated by the outstanding scientific legacy of the Apollo and Lunakhod programs. Future, currently planned sample return missions to the Moon (Sects. 2.2.2 and 2.2.3) will give us access to the broad diversity of Moon terrains, spanning their different ages. They raise the hope of a far better understanding of the formation history of the EarthMoon system and of a comprehensive inventory of the Moon's mineral and water resources. At Mars, sample return is the pillar of future exploration, bearing our hope to detect possible traces of extinct or extant life on the Red Planet (Sect. 3.2). Sample return from asteroids and comets, as the currently operating Hayabusa2 and OSIRIS-REx (Origins, Spectral Interpretation, Resource Identification, Security, Regolith Explorer) missions, will in parallel provide unique constraints on the initial composition of the building blocks of terrestrial planets and on their sources of water (Sect. 4). And while returning samples from the atmosphere or surface of Venus is probably one of the most challenging missions to fly, due to its very dense atmosphere, its huge scientific return on better understanding why Earth's sister planet is so different from its sibling fully justifies the required investment.

Finally, as the detection and characterisation of exoplanets is currently revolutionising the scope of planetary science, all means for assessing the composition of terrestrial exoplanets and their atmospheres will be extremely precious in order to place into a broader context the more accurate knowledge of the terrestrial planets composition in our Solar System (Sect. 5). Two methods are currently within the reach of past, planned and future exoplanet missions. The determination of the bulk composition of Ultra-short-period (USP) planets, in the range of Earth or super-Earth masses orbiting low-mass stars, provides precious constraints on their bulk composition and even on the existence of a significant atmosphere. In the longer term, missions dedicated to the spectroscopy of exoplanet atmospheres, using occultation or direct imaging, bear our greatest hopes to retrieve their origin and evolution. The most ambitious of these proposed missions (e.g., HabEx, LUVOIR and LIFE) open for us the perspective of detailed studies of habitable planets atmospheres, and hopes that inhabited ones might be discovered in a not-too-distant future. 

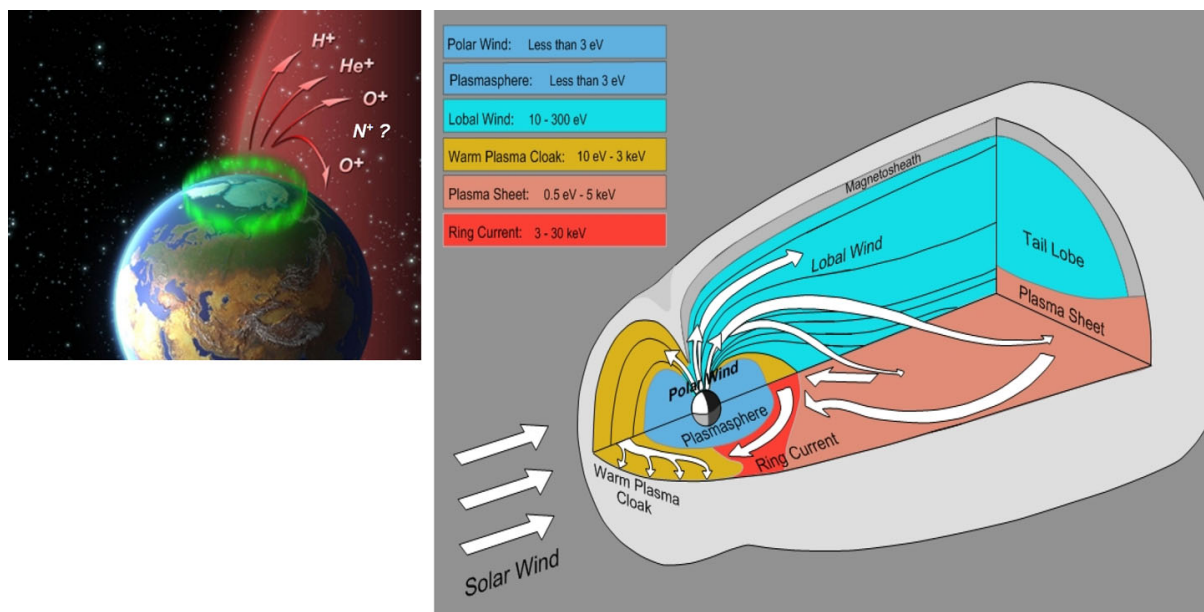

Fig. 1 Left: ion outflow from the terrestrial high-latitude ionosphere (adapted from Engwall 2009). Right: motion and energisation of outflowing low-energy ionospheric ions as they move through the magnetosphere. Depending on the outflow path, the interplanetary magnetic field conditions, the magnetospheric convection and the ion species, some of the ions are re-injected into the inner magnetosphere whereas some completely escape (from Chappell 2015)

\section{The Earth-Moon System}

\subsection{Earth}

The Earth is a unique habitable body with actual life, and that is strongly related to its atmosphere. However, the present atmospheric composition, following 4.5 billion years of evolution, is quite different from that of the ancient Earth (Stüeken et al. 2020). The evolution of the terrestrial atmosphere is driven by its interactions with the planet's crust and oceans, the biological activity, the influx from space (e.g., meteors) and the atmospheric escape to space (Yamauchi and Wahlund 2007; Lammer et al. 2008, 2011, 2018; Johnson and Goldblatt 2015; Airapetian et al. 2017a). The ions that outflow from the ionosphere (Fig. 1) are successively accelerated through a variety of energisation mechanisms and can eventually reach velocities above the gravitational escape velocity (e.g. Delcourt et al. 1993; Yau and André 1997; Huddleston et al. 2005; Nilsson et al. 2006, 2008). Depending on the outflow path, the interplanetary magnetic field conditions, the magnetospheric convection and the ion species, some of the outflowing ions are re-injected into the inner magnetosphere, whereas some completely escape (Ebihara et al. 2006; Haaland et al. 2012; Chappell 2015; Yamauchi 2019).

Ion outflow and atmospheric escape play an important role in the magnetospheric dynamics, by loading the magnetosphere with heavy ions (Kistler et al. 2005; Liu et al. 2013; Kronberg et al. 2014; Yamauchi and Slapak 2018), and in the evolution of the atmosphere on geological timescales, by slowly changing its composition (Ilie and Liemohn 2016; Lammer et al. 2018; Yamauchi 2019). They are highly dependent on the solar EUV flux and solar wind conditions and on the geomagnetic activity conditions, varying by 1 to 2 orders of magnitude (Slapak et al. 2017; Li et al. 2017; Schillings et al. 2019).

Earth being the most extensively explored body of our solar system, numerous missions since the beginning of the space era have been studying the upper atmosphere / ionosphere 
and the magnetosphere and have been collecting data on the ion and neutral sources, circulation within the magnetosphere and losses to outer space (e.g. Chappell 2015; Welling et al. 2015). However, several questions remain open, such as:

- What are the prevailing escape mechanisms and what are the escape rates, for ions and for neutrals, for different driver conditions (solar and geomagnetic activity), over geological time scales?

- How does the composition of the escaping populations change in response to different driver conditions, and in particular how does the nitrogen / oxygen escape ratio change as a function of the solar activity, over geological time scales?

- What is the isotope fractionation of the escaping fluxes and how it changes in response to different driver conditions?

- What is the degree of recirculation of plasma after it has left the ionosphere (recirculation versus escape)?

\subsubsection{Thermospheric / Exospheric Missions}

Table 1 provides a summary of the major past and present space missions, contributing to the study of the cold / thermal neutrals of the terrestrial thermosphere and exosphere (Dandouras et al. 2018).

It should be noted that for the extended exosphere these missions were mostly limited to hydrogen, whereas oxygen measurements were limited to altitudes below $\sim 700 \mathrm{~km}$ and for nitrogen below $\sim 600 \mathrm{~km}$, and these measurements are quite old (Johnson 1969). However, nitrogen and oxygen are the two major constituents of the Earth's atmosphere.

Regarding recent missions, the GOLD (Global-scale Observations of the Limb and Disk) mission of NASA was launched in 2018. It consists of a UV imaging spectrograph on a geostationary satellite providing remotely measured densities and temperatures in Earth's thermosphere for $\mathrm{O}$ and $\mathrm{N}_{2}$. Its objective is to study the global-scale response of the thermosphere and ionosphere to forcing in the integrated Sun-Earth system (https://gold.cs.ucf.edu/).

The ICON (Ionospheric Connection Explorer) mission of NASA was launched in October 2019 and will perform both in situ ion measurements, from its $575 \mathrm{~km}$ altitude orbit, i.e. close to the exobase, and remote-sensing measurements by pointing at the Earth's limb. These include an EUV and an FUV imager and a Michelson Interferometer for Global HighResolution Thermospheric Imaging. Its objectives focus on the ionosphere response to space weather activity (http://icon.ssl.berkeley.edu/).

Daedalus is a proposed mission to ESA, which has been selected for a Phase-0 study within the Earth Explorer Program. Its objective is to quantify the key electrodynamic processes that determine the structure and composition of the lower thermosphere (called also the "ignorosphere", or "agnostosphere"), the gateway between Earth's atmosphere and space, and its response to solar and geomagnetic activity conditions (Sarris et al. 2020). It relies on an innovative mission concept, with a main satellite on an elliptical $140 \times 2000 \mathrm{~km}$ altitude polar orbit, equipped with particle and wave instruments, including high-resolution ion and neutral mass spectrometers. The satellite will perform several short (day-long) excursions down to $\sim 120 \mathrm{~km}$. From there it could release each time an instrumented subsatellite (e.g. cubesat) whose altitude would gradually decay, providing in this way measurements in the heart of the most under-explored region in the Earth's atmosphere, until the sub-satellite burns in the mesosphere (https://daedalus.earth). Although its focus is the $100-200 \mathrm{~km}$ altitude range, it will be also capable of measuring ions and neutrals beyond the exobase better than DE-2. Daedalus will be in competition with two other missions, 
Table 1 Past and present thermospheric / exospheric missions

\begin{tabular}{|c|c|c|c|c|}
\hline Mission & Altitude range & $\begin{array}{l}\text { Remote sensing } \\
\text { method }\end{array}$ & $\begin{array}{l}\text { In situ method for } \\
\text { cold neutrals }\end{array}$ & Species \\
\hline $\begin{array}{l}\text { DE-2 } \\
(1981-1983)\end{array}$ & $\begin{array}{l}\text { thermosphere } \\
(300-500 \mathrm{~km}) \\
\text { apogee: } 1000 \mathrm{~km}\end{array}$ & & mass spectrometer & $\begin{array}{l}\text { major species } \\
\left(\mathrm{N}_{2}, \mathrm{~N}, \mathrm{O}, \mathrm{H}\right)\end{array}$ \\
\hline $\begin{array}{l}\text { AE-C, D, E } \\
(1973-1978)\end{array}$ & $\begin{array}{l}\text { thermosphere } \\
(<400 \mathrm{~km})\end{array}$ & UV & $\begin{array}{l}\text { mass spectrometer } \\
\text { accelerometer }\end{array}$ & $\begin{array}{l}\text { major species } \\
\left(\mathrm{N}_{2}, \mathrm{~N}, \mathrm{O}, \mathrm{H}\right)\end{array}$ \\
\hline $\begin{array}{l}\text { Air Force SCs } \\
(1968-)\end{array}$ & $\begin{array}{l}\text { thermosphere } \\
(<220 \mathrm{~km})\end{array}$ & & accelerometer & total mass density \\
\hline $\begin{array}{l}\text { Castor } \\
(1975-1979)\end{array}$ & $\begin{array}{l}\text { thermosphere } \\
(250-600 \mathrm{~km})\end{array}$ & & accelerometer & total mass density \\
\hline $\begin{array}{l}\text { San Marco } 5 \\
(1988,2 \text {-weeks })\end{array}$ & $\begin{array}{l}\text { thermosphere } \\
(260-690 \mathrm{~km})\end{array}$ & UV (not used) & accelerometer & total mass density \\
\hline $\begin{array}{l}\text { MSS } \\
(1980-1989)\end{array}$ & $\begin{array}{l}\text { thermosphere } \\
(<250 \mathrm{~km})\end{array}$ & UV occultation & & $\mathrm{O}_{2}$ density \\
\hline $\begin{array}{l}\text { TIMED } \\
(2001-)\end{array}$ & $\begin{array}{l}\text { thermosphere } \\
<400 \mathrm{~km} \\
\text { (target: }<180 \mathrm{~km} \text { ) }\end{array}$ & $\begin{array}{l}\text { multi-wavelength UV } \\
\text { (from } 600 \mathrm{~km} \text { ) }\end{array}$ & & $\begin{array}{l}\text { major species } \\
\left(\mathrm{N}_{2}, \mathrm{~N}, \mathrm{O}, \mathrm{H}\right)\end{array}$ \\
\hline $\begin{array}{l}\text { DEMETER } \\
(2004-2010)\end{array}$ & ionosphere & & & $\mathrm{H}^{+}, \mathrm{He}^{+}, \mathrm{O}^{+}$ \\
\hline $\begin{array}{l}\text { IMAGE } \\
(2000-2005)\end{array}$ & exosphere & Lyman- $\alpha$ & & H only \\
\hline $\begin{array}{l}\text { TWINS } \\
(2006-)\end{array}$ & exosphere & Lyman- $\alpha$ & & H only \\
\hline GOES & exosphere & $\begin{array}{l}\text { Lyman- } \alpha \\
\text { (from } 6.6 \mathrm{R}_{\mathrm{E}} \text { ) }\end{array}$ & & H only \\
\hline $\begin{array}{l}\text { GOLD } \\
(2018-)\end{array}$ & thermosphere & UV & & $\mathrm{O}, \mathrm{N}_{2}$ \\
\hline $\begin{array}{l}\text { ICON } \\
(2019-)\end{array}$ & thermosphere & EUV, FUV & RPA \& drift meter & \\
\hline
\end{tabular}

STEREOID (Cryosphere, Oceanography, Geosphere) and G-CLASS: $\mathrm{H}_{2} \mathrm{O}$ (rainfall, flooding landslides) for final selection within ESA's Earth Explorer Program. If selected, Daedalus launch is foreseen in 2027-2028.

AETHER (Aeronomy at Earth: Tools for Heliophysics Exploration and Research) is a mission proposed to NASA, which has been selected for a concept study. From a position on board the International Space Station, it would provide information on how the neutral, terrestrial-weather-driven thermosphere interacts with the ionosphere. AETHER will be in competition with two other missions, the Extreme Ultraviolet High-Throughput Spectroscopic Telescope (EUVST) and the Electrojet Zeeman Imaging Explorer (EZIE). If finally selected, AETHER would be launched in 2024.

\subsubsection{Magnetospheric Missions}

Table 2 provides a summary of the major past and present magnetospheric missions with composition measurements. 
Table 2 Major past and present magnetospheric missions

\begin{tabular}{|c|c|c|c|}
\hline Mission & Orbit & Key regions studied & Ion mass spectrometry \\
\hline $\begin{array}{l}\text { ISEE-1 \& ISEE-2 } \\
(1977-1987)\end{array}$ & $\begin{array}{l}6600 \mathrm{~km} \times 23 \mathrm{R}_{\mathrm{E}} \\
i \approx 29^{\circ}\end{array}$ & $\begin{array}{l}\text { Equatorial } \\
\text { magnetosphere }\end{array}$ & $\begin{array}{l}\text { SHM Ion Composition } \\
\text { Experiment }\end{array}$ \\
\hline $\begin{array}{l}\text { DE-1 } \\
(1981-1990)\end{array}$ & $\begin{array}{l}490 \mathrm{~km} \times 4.6 \mathrm{R}_{\mathrm{E}} \\
i \approx 90^{\circ}\end{array}$ & $\begin{array}{l}\text { Polar and Inner } \\
\text { Magnetosphere }\end{array}$ & $\begin{array}{l}\text { Retarding Ion Mass } \\
\text { Spectrometer (RIMS) }\end{array}$ \\
\hline $\begin{array}{l}\text { AMPTE / CCE } \\
(1984-1989)\end{array}$ & $\begin{array}{l}1100 \mathrm{~km} \times 9 \mathrm{R}_{\mathrm{E}} \\
i \approx 5^{\circ}\end{array}$ & $\begin{array}{l}\text { Equatorial inner } \\
\text { magnetosphere }\end{array}$ & $\begin{array}{l}\text { Hot Plasma Composition } \\
\text { Experiment (HPCE) \& } \\
\text { Charge-Energy-Mass } \\
\text { Spectrometer (CHEM) }\end{array}$ \\
\hline $\begin{array}{l}\text { Akebono } \\
(1989-2015)\end{array}$ & $\begin{array}{l}275 \mathrm{~km} \times 10500 \mathrm{~km} \\
i \approx 75^{\circ}\end{array}$ & Polar magnetosphere & $\begin{array}{l}\text { Suprathermal Ion Mass } \\
\text { Spectrometer (SMS) }\end{array}$ \\
\hline $\begin{array}{l}\text { Freja } \\
(1992-1996)\end{array}$ & $\begin{array}{l}600 \mathrm{~km} \times 1720 \mathrm{~km} \\
i \approx 63^{\circ}\end{array}$ & $\begin{array}{l}\text { Polar upper } \\
\text { ionosphere }\end{array}$ & $\begin{array}{l}\text { Three-dimensional Ion } \\
\text { Composition } \\
\text { Spectrometer (TICS) }\end{array}$ \\
\hline $\begin{array}{l}\text { SAMPEX } \\
(1992-2004)\end{array}$ & $\begin{array}{l}512 \mathrm{~km} \times 687 \mathrm{~km} \\
i \approx 82^{\circ}\end{array}$ & $\begin{array}{l}\text { Polar Inner } \\
\text { magnetosphere, } \\
\text { anomalous } \\
\text { components of } \\
\text { cosmic rays \& SEPs }\end{array}$ & $\begin{array}{l}\text { Low Energy Ion } \\
\text { Composition Analyser } \\
\text { (LEICA) \& Mass } \\
\text { Spectrometer Telescope } \\
\text { (MAST) }\end{array}$ \\
\hline $\begin{array}{l}\text { FAST } \\
(1996-2009)\end{array}$ & $\begin{array}{l}347 \mathrm{~km} \times 3500 \mathrm{~km} \\
i \approx 83^{\circ}\end{array}$ & Auroral region & $\begin{array}{l}\text { Time-of-flight Energy } \\
\text { Angle Mass Spectrograph } \\
\text { (TEAMS) }\end{array}$ \\
\hline $\begin{array}{l}\text { Geotail } \\
(1992-)\end{array}$ & $\begin{array}{l}9 \mathrm{R}_{\mathrm{E}} \times 31-220 \mathrm{R}_{\mathrm{E}} \\
i \approx 10^{\circ}\end{array}$ & Magnetotail & $\begin{array}{l}\text { Comprehensive Plasma } \\
\text { Investigation (CPI) \& } \\
\text { Energetic Particle and Ion } \\
\text { Composition Experiment } \\
\text { (EPIC) }\end{array}$ \\
\hline $\begin{array}{l}\text { Polar } \\
(1996-2008)\end{array}$ & $\begin{array}{l}4200 \mathrm{~km} \times 9 \mathrm{R}_{\mathrm{E}} \\
i \approx 79^{\circ}\end{array}$ & Polar magnetosphere & $\begin{array}{l}\text { Toroidal Imaging Mass } \\
\text { Angle Spectrograph } \\
\text { (TIMAS) \& Charge and } \\
\text { Mass Magnetospheric Ion } \\
\text { Composition Experiment } \\
\text { (CAMMICE) }\end{array}$ \\
\hline $\begin{array}{l}\text { Equator-S } \\
(1997-1998)\end{array}$ & $\begin{array}{l}212 \mathrm{~km} \times 35900 \mathrm{~km} \\
i \approx 4^{\circ}\end{array}$ & $\begin{array}{l}\text { Equatorial inner } \\
\text { magnetosphere }\end{array}$ & $\begin{array}{l}\text { Ion Composition } \\
\text { Instrument (ICI) }\end{array}$ \\
\hline $\begin{array}{l}\text { Cluster-4 spacecraft } \\
(2000-)\end{array}$ & $\begin{array}{l}4 \mathrm{R}_{\mathrm{E}} \times 19.6 \mathrm{R}_{\mathrm{E}} \\
i \approx 90^{\circ}\end{array}$ & $\begin{array}{l}\text { Magnetosphere, } \\
\text { magnetosheath, solar } \\
\text { wind }\end{array}$ & $\begin{array}{l}\text { Composition Distribution } \\
\text { Function (CODIF) \& } \\
\text { Research with Adaptive } \\
\text { Particle Imaging } \\
\text { Detectors (RAPID) }\end{array}$ \\
\hline $\begin{array}{l}\text { IMAGE } \\
(2000-2005)\end{array}$ & $\begin{array}{l}1000 \mathrm{~km} \times 8.2 \mathrm{R}_{\mathrm{E}} \\
i \approx 90^{\circ}\end{array}$ & $\begin{array}{l}\text { Ring current \& } \\
\text { plasmasphere } \\
\text { imaging }\end{array}$ & $\begin{array}{l}\text { Energetic neutral atom } \\
\text { imagers (LENA, MENA, } \\
\text { HENA) }\end{array}$ \\
\hline $\begin{array}{l}\text { Double Star TC1 \& TC2 } \\
(2004-2007)\end{array}$ & $\begin{array}{l}570 \mathrm{~km} \times 13.4 \mathrm{R}_{\mathrm{E}} \\
i \approx 28.5^{\circ}(\mathrm{TC} 1) \\
700 \mathrm{~km} \times 7.1 \mathrm{R}_{\mathrm{E}} \\
i \approx 90^{\circ}(\mathrm{TC} 2)\end{array}$ & $\begin{array}{l}\text { Equatorial } \\
\text { magnetosphere (TC1) } \\
\& \text { Polar } \\
\text { magnetosphere (TC2) }\end{array}$ & $\begin{array}{l}\text { Hot Ion Analyser (indirect } \\
\text { composition) }\end{array}$ \\
\hline $\begin{array}{l}\text { TWINS A \& B } \\
(2006-)\end{array}$ & $\begin{array}{l}1000 \mathrm{~km} \times 7.2 \mathrm{R}_{\mathrm{E}} \\
i \approx 63^{\circ}\end{array}$ & $\begin{array}{l}\text { Ring current \& } \\
\text { exosphere imaging }\end{array}$ & $\begin{array}{l}\text { Energetic neutral atom } \\
\text { imager (MENA) }\end{array}$ \\
\hline
\end{tabular}


Table 2 (Continued)

\begin{tabular}{|c|c|c|c|}
\hline Mission & Orbit & Key regions studied & Ion mass spectrometry \\
\hline $\begin{array}{l}\text { THEMIS-5 spacecraft } \\
(2007-)\end{array}$ & $\begin{array}{l}470 \mathrm{~km} \times 12-16 \mathrm{R}_{\mathrm{E}} \\
i \approx 16^{\circ}\end{array}$ & $\begin{array}{l}\text { Magnetosphere, } \\
\text { magnetosheath, solar } \\
\text { wind }\end{array}$ & $\begin{array}{l}\text { Electrostatic Analyser } \\
\text { (indirect composition) }\end{array}$ \\
\hline $\begin{array}{l}\text { Van Allen Probes A \& B } \\
(2012-2019)\end{array}$ & $\begin{array}{l}618 \mathrm{~km} \times 30400 \mathrm{~km} \\
i \approx 10^{\circ}\end{array}$ & Inner Magnetosphere & $\begin{array}{l}\text { Ion Composition } \\
\text { Experiment (RBSPICE) \& } \\
\text { Energetic Particle, } \\
\text { Composition, and } \\
\text { Thermal Plasma (ECT) }\end{array}$ \\
\hline $\begin{array}{l}\text { MMS-4 spacecraft } \\
(2015-)\end{array}$ & $\begin{array}{l}2550 \mathrm{~km} \times 12-25 \mathrm{R}_{\mathrm{E}} \\
i \approx 63^{\circ}\end{array}$ & $\begin{array}{l}\text { Magnetosphere, } \\
\text { magnetosheath, solar } \\
\text { wind }\end{array}$ & $\begin{array}{l}\text { Hot Plasma Composition } \\
\text { Analyser (HPCA) \& } \\
\text { Energetic Ion } \\
\text { Spectrometer (EIS) }\end{array}$ \\
\hline $\begin{array}{l}\text { ERG / Arase } \\
(2016-)\end{array}$ & $\begin{array}{l}400 \mathrm{~km} \times 32100 \mathrm{~km} \\
i \approx 31^{\circ}\end{array}$ & Inner Magnetosphere & $\begin{array}{l}\text { Low-Energy Particle } \\
\text { Experiment-Ion Mass } \\
\text { Analyser (LEP-i) \& } \\
\text { Medium-Energy Particle } \\
\text { Experiment-Ion Mass } \\
\text { Analyser (MEP-i) }\end{array}$ \\
\hline
\end{tabular}

The above list is not comprehensive, but focuses on the major missions equipped to supply ion composition information.

Currently, the Cluster mission of ESA, launched in 2000 (Escoubet et al. 2001, 2015), and the MMS (Magnetospheric Multiscale Mission) mission of NASA, launched in 2015 (Burch et al. 2016), are the two major missions supplying in situ magnetospheric ion composition measurements. Each of these missions involves four spacecraft, providing thus threedimensional measurements of space plasma parameters. These missions have been very successful in analysing medium-scale (Cluster) and small-scale (MMS) plasma dynamics. They have been also supplying key information on plasma sources and losses (e.g. Engwall et al. 2009; Dandouras 2013; Parks et al. 2015; Nagai et al. 2016; Slapak et al. 2017; $\mathrm{Xu}$ et al. 2019). However, the moderate mass resolution of their instrumentation $(m / \Delta m \approx$ 5-7 for CODIF onboard Cluster (Rème et al. 2001) and $m / \Delta m \approx 4$ for HPCA on board MMS (Young et al. 2016)) does not allow distinguishing nitrogen from oxygen ions or any isotope ratios. The separation of nitrogen from oxygen was designed for DE-1, AMPTE, Akebono and Arase, but for limited energy ranges, and such separation is partially possible for the Van Allen Probes.

ESCAPE (European SpaceCraft for the study of Atmospheric Particle Escape) is a mission that has been proposed to ESA, in response to the M5 call, to quantitatively estimate the amount of escaping particles of the major atmospheric components (nitrogen and oxygen), as neutral and ionised species, escaping from the Earth as a magnetised planet. The goal is to understand the importance of each escape mechanism (thermal or non-thermal), its dependence on solar and geomagnetic activity, in order to infer the history of the Earth's atmosphere over a long (geological scale) time period. To achieve this goal a slowly spinning spacecraft on a high inclination $500 \mathrm{~km} \times 33000 \mathrm{~km}$ orbit has been proposed, equipped with a suite of instruments for both in situ measurements and for remote-sensing observations (Dandouras et al. 2018). This instrument suite would provide high-mass resolution measurements, providing nitrogen/oxygen separation and isotope fractionation over a wide energy range (http://cluster.irap.omp.eu/public/ESCAPE/ESCAPE_M5_Proposal_V1.1.pdf). 
In March 2017 ESCAPE successfully passed the first technical and programmatic screening by ESA, but then in May 2018 ESCAPE was not selected for flight.

MEME-X (Mechanisms of Energetic Mass Ejection - eXplorer) is another proposed mission, that was selected in 2017 by NASA for a competitive study within the Small Explorer program (SMEX). Based on two spacecraft flying in twin $350 \times 1200 \mathrm{~km}$ polar orbits, it would study how plasma escapes from the ionosphere into the magnetosphere (https://www.nasa.gov/feature/goddard/2017/new-nasa-mission-concept-aimed-atstudying-why-planets-lose-their-atmospheres). In June 2019 MEME-X was not selected for flight.

SMILE (Solar wind Magnetosphere Ionosphere Link Explorer) is a joint mission between ESA and the Chinese Academy of Sciences, currently under preparation and scheduled for launch in 2023 (http://sci.esa.int/smile/). Its objective is to investigate the dynamic response of the magnetosphere to the solar wind conditions in a global way. It will carry four instruments. Two of them will be for in situ measurements: a light ion analyser and a magnetometer. The other two will be imagers: an ultraviolet aurora imager and a novel soft X-ray imager. This imager will observe the magnetosheath, the magnetopause and the cusps, and their response to solar wind conditions, through the X-rays emitted from charge exchange between solar wind highly charged ions and exospheric neutrals (Sibeck et al. 2018). SMILE will be placed on a highly inclined elliptical orbit with an apogee at $19 R_{E}$, enabling the spacecraft to spend most of its time at high altitudes, so as to collect continuous observations through imaging for long periods.

\subsection{Moon}

The Moon, during most part of its orbit around the Earth is directly exposed to the solar wind. Due to the absence of a substantial intrinsic magnetic field and of a collisional atmosphere, solar wind and solar energetic particles (SEPs) arrive almost without any deviation or absorption and impact directly on its surface, interacting with the lunar regolith and the tenuous lunar exosphere (e.g. Geiss et al. 2004; Wurz et al. 2007; Futaana et al. 2018). The same phenomenon occurs also with the galactic cosmic rays (GCRs), which present fluxes and energy spectra typical of the interplanetary space (e.g. Sohn et al. 2014).

During 5-6 days every orbit, however, the Moon crosses the tail of the terrestrial magnetosphere (Fig. 2). It is then exposed not to the solar wind but to the terrestrial magnetotail plasma environment, offering the possibility to study in situ magnetotail dynamics and its dependence on solar and geomagnetic activity (e.g. Kallio and Facskó 2015; Kallio et al. 2019). Phenomena such as plasmoids released from the near-Earth magnetotail and propagating anti-Sunward, bursty bulk flows (BBFs), magnetic reconnection and plasma sheet dynamics can be studied in situ (e.g. Nakamura 2006; Cao et al. 2013). The Moon is then also very well situated to study atmospheric escape from the Earth into space (Lammer et al. 2008), in the form of heavy ions upwelling from the terrestrial ionosphere and then transported and lost into the deep magnetotail, as shown from the wealth of data supplied from the THEMIS-ARTEMIS instruments (cf. Sect. 2.2.1) and from the Kaguya and Chandrayaan-1 spacecraft (Poppe et al. 2016; Terada et al. 2017; Dandouras et al. 2020; Li et al. 2020). The THEMIS-ARTEMIS data, however, did not include the crucial information on the plasma composition (Angelopoulos 2011), and the Kaguya plasma measurements where limited to a less than two-year mission and to low-energy plasma (Saito et al. 2010). Far magnetotail studies performed by the Geotail spacecraft supplied crucial information on the dynamics of ion beams streaming downtail (Christon et al. 1994; 
Fig. 2 Moon's orbit with respect to the Earth's magnetosphere. Earth's and Moon's sizes are not in scale (adapted from: Tim Stubbs / University of Maryland / GSFC

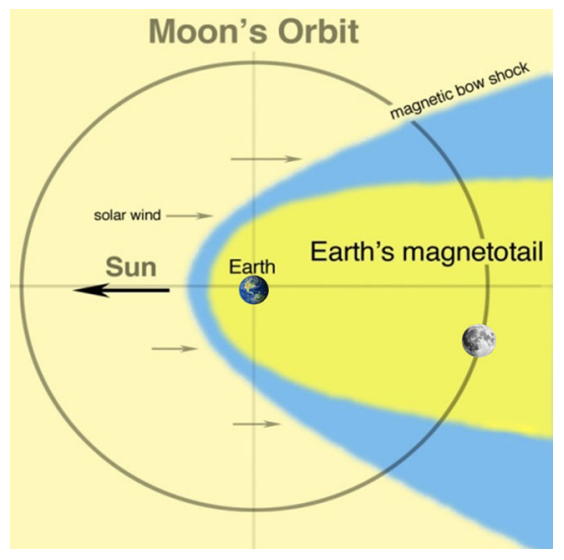

Seki et al. 1998), but lacked ion composition measurements at low energies (below $\sim 10 \mathrm{keV}$ ).

But, most important, the lunar environment offers a unique opportunity to study the Moon surface-bounded exosphere, its production mechanisms, its dynamics, its interaction with the solar wind and with the terrestrial magnetotail plasma, and its escape into space (Potter et al. 2000; Wurz et al. 2007; Leblanc and Chaufray 2011; Harada et al. 2014). The LADEE (Lunar Atmosphere and Dust Environment Explorer) observations have just shown the complexity of the lunar exosphere and of the associated physical mechanisms (Elphic et al. 2014; Benna et al. 2015; Hodges 2016).

The Moon surface also offers exciting possibilities for studying energetic ion implantation in the lunar regolith (Ozima et al. 2005; Ireland et al. 2006), solar wind ion implantation or neutralisation and reflection from the lunar regolith (Vorburger et al. 2015; Tucker et al. 2019), solar wind interaction with crustal magnetic anomalies (Vorburger et al. 2012; Poppe et al. 2015; Bamford et al. 2016), lunar pick-up ion generation (Poppe et al. 2012), or lunar surface electrostatic charging and dust levitation (Stubbs et al. 2007; Burinskaya 2015; Dyadechkin et al. 2015), just to mention few examples.

Table 3 provides a summary of the major past and present lunar missions with composition measurements.

Following the 50th anniversary of the Apollo missions to the Moon, and the more recent missions mentioned above (THEMIS-ARTEMIS, Kaguya, LADEE), a series of lunar missions is in preparation, or already operating, building on their outstanding legacy.

\subsubsection{Orbiters}

The THEMIS-ARTEMIS P1 and P2 NASA spacecraft entered lunar orbit on 27 June and 17 July of 2011 respectively, after they have been exploring the terrestrial magnetosphere since 2007 (Angelopoulos 2011). Each satellite carries identical instrumentation, including a fluxgate magnetometer (FGM), an electrostatic analyser (ESA), a solid state telescope (SST), a search-coil magnetometer (SCM) and an electric field instrument (EFI).

The Lunar Reconnaissance Orbiter (LRO) of NASA was launched on 18 June 2009 (Keller et al. 2016). The scientific payload consists of: (i) a Cosmic Ray Telescope for the Effects of Radiation (CRaTER) to characterise the effects of radiation in the lunar environment and its biological impacts; (ii) a Diviner Lunar Radiometer Experiment to measure the lunar 
Table 3 Major past and present lunar missions with composition measurements

\begin{tabular}{|c|c|c|}
\hline Mission & Mission type & Composition measurements \\
\hline $\begin{array}{l}\text { Luna } 11 \\
(1966)\end{array}$ & Lunar orbiter & $\gamma$-ray spectrometer \\
\hline $\begin{array}{l}\text { Apollo } 11-14 \\
(1969-1971)\end{array}$ & $\begin{array}{l}\text { Human } \\
\text { spaceflight: } \\
\text { orbiter + lander }\end{array}$ & $\begin{array}{l}\text { Lunar sample return \& } \\
\text { Solar wind composition experiment }\end{array}$ \\
\hline $\begin{array}{l}\text { Luna } 16 \\
(1970)\end{array}$ & Lunar lander & Lunar sample return \\
\hline $\begin{array}{l}\text { Apollo } 15-17 \\
(1971-1972)\end{array}$ & $\begin{array}{l}\text { Human } \\
\text { spaceflight: } \\
\text { orbiter + lander } \\
\text { + subsatellite }\end{array}$ & $\begin{array}{l}\text { Lunar sample return \& } \\
\text { Solar wind composition experiment \& } \\
\text { Particles and fields subsatellite }\end{array}$ \\
\hline $\begin{array}{l}\text { Luna } 19 \& 22 \\
(1971-1972,1974-1975)\end{array}$ & Lunar orbiter & $\gamma$-ray spectrometer \\
\hline $\begin{array}{l}\text { Luna } 20 \& 24 \\
(1972,1976)\end{array}$ & Lunar lander & Lunar sample return \\
\hline $\begin{array}{l}\text { Geotail } \\
(1992-)\end{array}$ & $\begin{array}{l}\text { Earth orbiter }+ \\
\text { Lunar flybys }\end{array}$ & $\begin{array}{l}\text { Comprehensive Plasma Investigation \& } \\
\text { Energetic Particle and Ion Composition } \\
\text { Experiment }\end{array}$ \\
\hline $\begin{array}{l}\text { Clementine } \\
(1994-1995)\end{array}$ & Lunar orbiter & Multi-spectral imaging of lunar surface \\
\hline $\begin{array}{l}\text { Lunar Prospector } \\
(1998-1999)\end{array}$ & Lunar orbiter & $\begin{array}{l}\gamma \text {-ray spectrometer } \& \\
\text { Neutron spectrometer } \& \\
\alpha \text { particle spectrometer }\end{array}$ \\
\hline $\begin{array}{l}\text { SMART-1 } \\
(2003-2006)\end{array}$ & Lunar orbiter & X-ray spectrometer \\
\hline $\begin{array}{l}\text { SELENE (Kaguya) } \\
(2007-2009)\end{array}$ & Lunar orbiter & $\begin{array}{l}\gamma \text {-ray spectrometer } \& \\
\text { X-ray spectrometer } \& \\
\text { Charged particle spectrometer }\end{array}$ \\
\hline $\begin{array}{l}\text { Chang'e } 1 \\
(2007-2009)\end{array}$ & Lunar orbiter & $\begin{array}{l}\gamma \text {-ray spectrometer \& } \\
\mathrm{X} \text {-ray spectrometer \& Imaging } \\
\text { spectrometer }\end{array}$ \\
\hline $\begin{array}{l}\text { Chandrayaan-1 } \\
(2008-2009)\end{array}$ & Lunar orbiter & $\begin{array}{l}\text { High-Energy X-ray spectrometer \& } \\
\text { Hyper spectral imaging camera \& } \\
\text { Visible near-IR imaging spectrometer \& } \\
\text { ENA analyser }\end{array}$ \\
\hline $\begin{array}{l}\text { Lunar Reconnaissance Orbiter (LRO) } \\
(2009-)\end{array}$ & Lunar orbiter & $\begin{array}{l}\text { Lyman- } \alpha \text { Mapping \& } \\
\text { Lunar Exploration Neutron Detector }\end{array}$ \\
\hline $\begin{array}{l}\text { Lunar Crater Observation and } \\
\text { Sensing Satellite (LCROSS) } \\
(2009)\end{array}$ & Lunar impactor & Multi-spectral cameras \\
\hline $\begin{array}{l}\text { Chang'e } 2 \\
(2010-2011)\end{array}$ & Lunar orbiter & $\begin{array}{l}\gamma \text {-ray spectrometer } \& \\
\mathrm{X} \text {-ray spectrometer } \& \\
\text { Imaging spectrometer }\end{array}$ \\
\hline $\begin{array}{l}\text { THEMIS-ARTEMIS P1 \& P2 } \\
(2011-)\end{array}$ & Lunar orbiter & $\begin{array}{l}\text { Electrostatic Analyser (indirect } \\
\text { composition) }\end{array}$ \\
\hline $\begin{array}{l}\text { Lunar Atmosphere and Dust } \\
\text { Environment Explorer (LADEE) } \\
(2013-2014)\end{array}$ & Lunar orbiter & $\begin{array}{l}\text { Neutral Mass Spectrometer \& } \\
\text { UV-Visible Spectrometer }\end{array}$ \\
\hline
\end{tabular}


Table 3 (Continued)

\begin{tabular}{|c|c|c|}
\hline Mission & Mission type & Composition measurements \\
\hline $\begin{array}{l}\text { Chang'e } 3 \\
(2013-2015)\end{array}$ & $\begin{array}{l}\text { Lunar lander + } \\
\text { rover }\end{array}$ & $\begin{array}{l}\text { Ground-penetrating radar \& } \\
\alpha \text { particle X-ray spectrometer }\end{array}$ \\
\hline $\begin{array}{l}\text { Chang'e } 4 \\
(2018-)\end{array}$ & $\begin{array}{l}\text { Lunar lander + } \\
\text { rover + relay } \\
\text { satellite }\end{array}$ & $\begin{array}{l}\text { Ground-penetrating radar \& } \\
\alpha \text {-particle X-ray spectrometer }\end{array}$ \\
\hline $\begin{array}{l}\text { Chandrayaan-2 } \\
(2019-)\end{array}$ & Lunar orbiter & $\begin{array}{l}\text { Soft X-ray spectrometer \& } \\
\text { Imaging IR Spectrometer \& } \\
\text { Atmospheric composition analyser }\end{array}$ \\
\hline
\end{tabular}

surface thermal emission and to provide information for future surface operations and exploration; (iii) the Lyman-Alpha Mapping Project to look into permanently shadowed craters in search of water ice, using ultraviolet light; (iv) the Lunar Exploration Neutron Detector to measure and map neutrons, in search of possible near-surface water ice deposits; (v) the Lunar Orbiter Laser Altimeter to produce a precise global lunar topographic model and geodetic grid; (vi) the Lunar Reconnaissance Orbiter Camera to address the requirements of landing site certification and polar illumination; (vii) the Miniature Radio Frequency radar to demonstrate new lightweight SAR and communications technologies and locate potential water-ice.

The Hakuto-R mission 1 of ispace inc. (Japan) is a commercial lunar exploration program (https://ispace-inc.com/hakuto-r/). Mission 1 is scheduled for launch in 2021 and will perform a soft landing on the Moon. Mission 2, scheduled for launch in 2023, will perform a soft landing and deploy a rover for surface exploration and data collection on the Moon.

The Deep Space Gateway (or Lunar Orbital Platform - Gateway, cf. Fig. 3) is a crewed platform that will be assembled and operated in the vicinity of the Moon by NASA, ESA, JAXA, Roscosmos and the CSA, and will offer new opportunities for fundamental and applied scientific research (Carpenter et al. 2018). Deployment of its different modules is planned within the 2022-2028 timeframe, and early utilisation for scientific payloads will be possible as of 2024 (Gateway Phase 1). In 2027 the ESPRIT (European System Providing Refuelling, Infrastructure and Tele-communication) module will also be available (Gateway Phase 2). Space plasma physics measurements could be performed either from the Deep Space Gateway platform, or from instrumented CubeSats released from the platform and placed into low-lunar orbits, or directly from the Moon surface (Dandouras et al. 2019).

\subsubsection{Orbiters with Landers}

The Chang'E-5 mission of CNSA is scheduled for launch in 2020, with a chosen landing site near Mons Rümker, an isolated volcanic formation in Oceanus Procellarum. Chang'E-5 has also a sample-return vehicle to return back to the Earth at least 2 kilograms of lunar soil and rock samples, including a 2-metre drill sample of the lunar regolith. The lander will carry landing cameras, a panoramic camera, a mineral spectrometer, a soil gas analytical instrument, a soil composition analytical instrument, a sampling sectional thermo-detector, and a ground-penetrating radar. For acquiring samples, it will be equipped with a robotic arm, a rotary-percussive drill, a scoop for sampling, and separation tubes to isolate individual samples. The planned landing area is one of the youngest regions on the Moon $(\sim 1.5 \mathrm{Ga})$, hence the returned samples will shed light on the final volcanic eruptions and thermal history of the Moon and determine the impact cratering rate between $\sim 1$ to $\sim 3 \mathrm{Ga}$. 


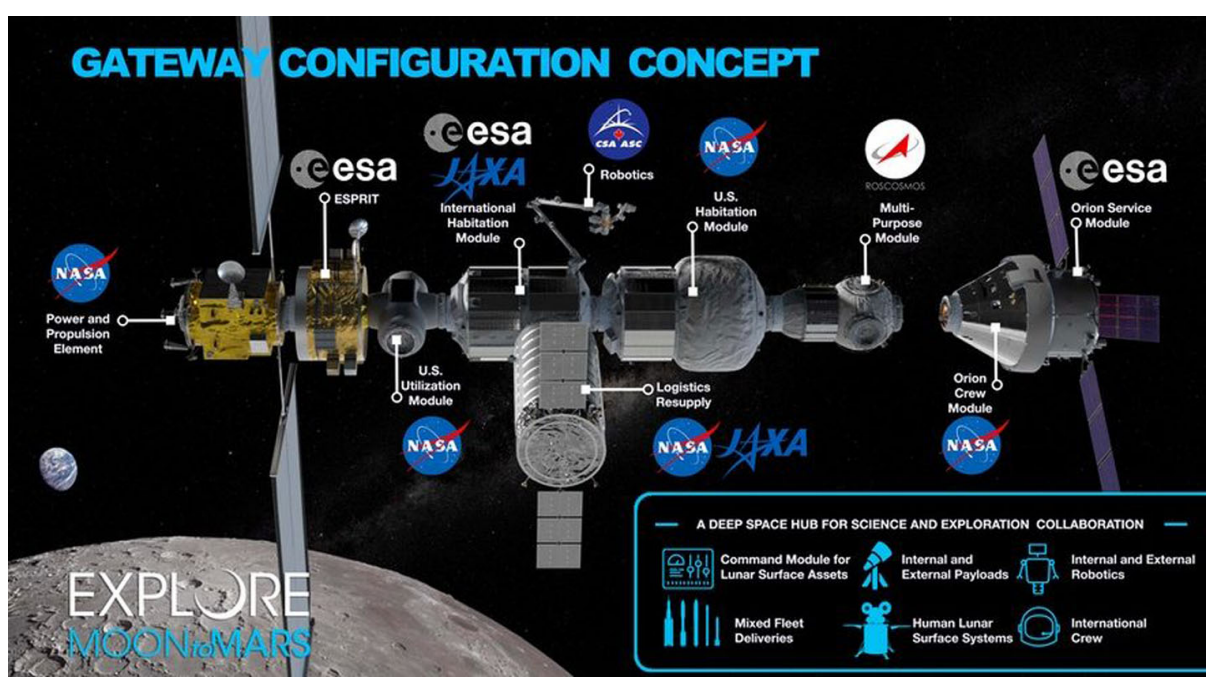

Fig. 3 Illustration showing the Deep Space Gateway (or Lunar Orbital Platform - Gateway) configuration, and the proposed international partners. Credit: NASA

The Chang'E-6 mission of CNSA is scheduled for launch in 2023-2024 and will perform China's second sample return mission. Chang'E-6 is the backup of Chang'E-5. If Chang'E5 is successful, Chang'E-6 will be considered as a part of the 4th stage of the Chang'E lunar exploration program, which contains also two other landing missions (numbered as Chang'E-7 and 8). Chang'E-6 is planned to land in the south polar region of the Moon and bring back samples from there, including materials delivered from the SPA basin (cf. also Sect. 2.2.4).

\subsubsection{Landers}

The OMOTENASHI (Outstanding MOon exploration TEchnologies demonstrated by NAno Semi-Hard Impactor) mission of JAXA (via the Exploration Mission 1, or EM-1, of NASA) is a small spacecraft and semi-hard lander of the $6 \mathrm{U}$ CubeSat format that will demonstrate low-cost technologies to land and explore the lunar surface. The CubeSat will also take measurements of the radiation environment near the Moon as well as on the lunar surface.

The Peregrine mission of Astrobotic, USA (https://www.astrobotic.com/lunar-delivery), is scheduled to be launched in 2021 and to land at Lacus Mortis. The Peregrine Lander can accommodate a variety of payload types for science, exploration, resources etc.

The XL 1 mission of Masten Space System, USA (https://www.masten.aero/lunar-vehicles), is scheduled to be launched in 2021 and to land at the Aristarchus Crater. It has been designed in partnership with the NASA Lunar CATALYST program (public-private partnership) and is capable of placing a 100-kg payload on the lunar surface.

The Smart Lander for Investigating Moon (SLIM) mission of JAXA is scheduled to be launched in 2021-2022 and to land in the Mare Tranquillitatis. SLIM also has a samplereturn vehicle to bring back a sample of about the size of the Hayabusa capability.

Luna-25 (Luna-Glob) and Luna-27 (Luna-Resurs) are two planned missions to send a lander to the South Pole-Aitken basin, under preparation by Roscosmos in collaboration with ESA. Their objectives include the detection and characterisation of lunar polar volatiles (Sanin and Mitrofanov 2016). 
HERACLES (Human-Enhanced Robotic Architecture and Capability for Lunar Exploration and Science) is a robotic lunar transport system planned by ESA, JAXA and the CSA. It will feature the European Large Logistic Lander (EL3), designed to allow a series of different missions with different options for its payloads. The project is exploration-driven with a strong scientific content in a variety of domains, as samples return, polar ice and volatiles, internal geophysics, space plasma environment, radiation biological effects, radio astronomy, or identification of in situ resources (https://www.esa.int/Science_Exploration/Human_and_ Robotic_Exploration/Exploration/European_Large_Logistics_Lander).

\subsubsection{Landers and Rovers}

In January 2019 the Chinese Chang' E-4 mission, with its relay satellite Queqiao, involving a robotic lander and the Yutu-2 rover, performed the first landing on the far-side of the Moon. The initial results of this ongoing mission include the spectroscopic identification of lunar far-side mantle-derived materials (Li et al. 2019) and the thick regolith and complex subsurface structures detected by the on-board Lunar Penetrating Radar (LPR).

Together with Chang'E-6, both Chang'E-7 and 8 missions of CNSA are the main parts of the 4th stage of the Chang'E program. All of these three missions aim to land in the south polar region of the Moon, and will establish a prototype lunar research station by the end of the 4th stage of Chang'E program. Chang'E-7 and 8 are scheduled for launch before and after Chang'E-6, respectively. The primary lunar science objectives of these missions are to explore in situ the possible water ice in the permanently shadowed craters, detect the lunar interior structures by delivering lunar seismometers, determine the volatile components in the lunar regolith in the polar region and explore the polar geology, geochemistry and space environment.

The Indian Chandrayaan-2 mission, launched on 22 July 2019, is composed of a lunar orbiter, a lander and a rover. Its scientific objectives include lunar topography, mineralogy, elemental abundance, the lunar exosphere, and signatures of hydroxyl and water ice (Goswami 2014). Communications with the lander were lost when touchdown was expected, on 7 September 2019, but the orbiter, equipped with eight scientific instruments, is operational and is expected to continue its seven-year mission to study the Moon.

The NASA VIPER (Volatiles Investigating Polar Exploration Rover) project consists of a mobile robot that will be sent to the South Pole of the Moon in December 2022. There it will get a close-up view of the location and concentration of water ice in the region and will, for the first time, actually sample the water ice at the same pole where the first woman and next man will land in 2024 under the Artemis program. VIPER will roam several km, using its four instruments, including a 1-meter drill, to sample various soil environments. It will collect about 100 days of data that will then be used to prepare the first global water resource maps of the Moon.

\section{Noble Gas Atmosphere Missions}

\subsection{Venus}

Our sister planet Venus attracts a large interest due to its very dense atmosphere, which shows drastically different evolutionary paths compared to Earth (Lammer et al. 2020c). Starting from the early 1960's multiple missions to Venus were accomplished by the USSR, USA and Europe, the most recent of which were Venus-Express (ESA, 2006-2015) and 
Table 4 Major past and present Venus missions with composition measurements

\begin{tabular}{|c|c|c|}
\hline Mission & Mission type & Composition measurements \\
\hline $\begin{array}{l}\text { Venera } 4,5,6 \\
(1967,1969,1969)\end{array}$ & $\begin{array}{l}\text { Venus orbiter + entry } \\
\text { probe }\end{array}$ & Atmospheric composition analyser \\
\hline $\begin{array}{l}\text { Venera } 8 \\
(1972)\end{array}$ & $\begin{array}{l}\text { Venus orbiter }+ \text { entry } \\
\text { probe / lander }\end{array}$ & $\begin{array}{l}\text { Atmospheric composition analyser \& } \\
\gamma \text {-ray spectrometer }\end{array}$ \\
\hline $\begin{array}{l}\text { Mariner } 10 \\
(1974)\end{array}$ & $\begin{array}{l}\text { Interplanetary probe }+ \\
\text { Venus flyby }\end{array}$ & UV spectrometer \& IR radiometer \\
\hline $\begin{array}{l}\text { Venera } 9,11,12 \\
(1975,1978,1978)\end{array}$ & $\begin{array}{l}\text { Venus orbiter + entry } \\
\text { probe / lander }\end{array}$ & $\begin{array}{l}\text { Atmospheric composition analyser \& } \\
\gamma \text {-ray spectrometer \& Imager }\end{array}$ \\
\hline $\begin{array}{l}\text { Pioneer Venus Orbiter } \\
\text { (Pioneer Venus 1) } \\
(1978-1992)\end{array}$ & Venus orbiter & $\begin{array}{l}\text { UV spectrometer \& } \\
\text { Neutral mass spectrometer \& } \\
\text { Ion mass spectrometer }\end{array}$ \\
\hline $\begin{array}{l}\text { Pioneer Venus Multiprobe } \\
\text { (Pioneer Venus 2) } \\
\text { (1978) }\end{array}$ & $\begin{array}{l}\text { Venus orbiter }+ \text { entry } \\
\text { probes }\end{array}$ & $\begin{array}{l}\text { Ion mass spectrometer } \& \\
\text { Neutral mass spectrometer } \& \\
\text { Gas chromatograph }\end{array}$ \\
\hline $\begin{array}{l}\text { Venera } 13,14 \\
(1982)\end{array}$ & $\begin{array}{l}\text { Interplanetary probe }+ \\
\text { Venus lander }\end{array}$ & $\begin{array}{l}\text { Neutral mass spectrometer \& } \\
\text { Gas chromatograph \& } \\
\text { X-ray fluorescence spectrometer }\end{array}$ \\
\hline $\begin{array}{l}\text { Venera } 15,16 \\
(1983-1985)\end{array}$ & Venus orbiter & IR Fourier spectrometer \\
\hline $\begin{array}{l}\text { Vega } 1,2 \\
(1985)\end{array}$ & $\begin{array}{l}\text { Interplanetary probe }+ \\
\text { Venus lander }+ \text { Balloon }\end{array}$ & $\begin{array}{l}\text { UV spectrometer \& } \\
\text { Aerosol mass spectrometer \& } \\
\text { Aerosol gas chromatograph \& } \\
\text { Aerosol X-ray spectrometer \& } \\
\gamma \text {-ray spectrometer }\end{array}$ \\
\hline $\begin{array}{l}\text { Venus Express } \\
(2006-2015)\end{array}$ & Venus orbiter & $\begin{array}{l}\text { Analyser of plasma and ENAs \& } \\
\text { IR spectrometer \& } \\
\text { Visible and IR imaging spectrometer }\end{array}$ \\
\hline $\begin{array}{l}\text { Akatsuki } \\
\text { (Venus Climate Orbiter) } \\
(2015-)\end{array}$ & Venus orbiter & UV imager \\
\hline
\end{tabular}

Akatsuki (JAXA, 2010- ). Table 4 provides a summary of the major past and present missions to Venus with composition measurements.

There is still a question on how Venus could have lost its water and acquire a dense greenhouse-driven $\mathrm{CO}_{2}$ atmosphere. Measurements of the abundance and of the isotopic composition of noble gases are a key element to understand the evolution of the atmosphere. This can be done only by direct measurements on board atmospheric probes. The latest successful landing on Venus was that of the Vega-2 lander in 1985 and many key parameters, including noble gases composition, need more precise measurements by modern instruments.

Russia is planning to launch a new mission to Venus in the late 2020s with the name Venera-D (D is an abbreviation of the Russian word "dolgozhivuschaya", that means long leaving). The mission is still at a level of planning and has a high probability to be included into the national space program. Following the intention of NASA to join the Venera-D mission, the space agencies of both countries established the JSDT (Joint Science Definition Team) bringing together 22 members for the formulation of the shape of the mission. Venera$\mathrm{D}$ is planned to be a comprehensive investigation of Venus with three baseline elements: the 
orbiter, the lander and LLISSE (Long Lived In situ Solar System Explorer) (Report of the Venera-D JSDT, 2019).

The Russian-built orbiter is planned to have about $100 \mathrm{~kg}$ of science payload which includes a Fourier Spectrometer, an Ultraviolet mapping spectrometer, a Visible-to-Mid Infrared Spectrometer, an Ultraviolet-Near Infrared Imaging, a Solar and star occultation spectrometer, a Millimeter-radiometer, an Infrared heterodyne spectrometer, a Thermal Infrared Camera, an Infrared Imaging Camera, a Radio-package for two-frequency occultation in Land X-bands, an Electromagnetic waves detector, and a Panoramic energy mass-analyser of ions. The scientific objectives of the orbiter are: a) study the dynamics and nature of superrotation, radiative balance, and the nature of the greenhouse effect; b) characterise the thermal structure of the atmosphere, winds, thermal tides, and solar-locked structures; c) measure the atmospheric composition, study the clouds, their structure, composition, microphysics, and chemistry; d) investigate the upper atmosphere, ionosphere, electrical activity, magnetosphere, atmospheric escape rate and solar wind interaction. The mission scenario would place the spacecraft in a highly inclined, near-polar orbit with a period of 24 hours. The expected operational lifetime of the orbiter would be more than 3 years.

The Russian-built Lander is planned to be based on the Vega heritage, with about 100$120 \mathrm{~kg}$ of science payload operating for about 1 hour during the descent and up to 3 hours at the surface. The scientific payload will include: an X-Ray Diffraction / X-Ray Fluorescent Analyser (XRD/XRF), a Mössbauer spectrometer with XRF mode, TV- cameras (landing, stereo, panoramic, high resolution up to $0.1 \mathrm{~mm}$ ), a Chemical Analysis Package which will be an assemblage of Gas Chromatograph-Mass-Spectrometer-Laser-Induced MassSpectrometer (GCMS + LIMS), a Raman Spectrometer (with synergistic LIDAR), a Meteo Package, an Active Gamma and Neutron Spectrometers, a Multi-channel Tunable Diode Laser Absorption Spectrometer (MTDLAS), and an Infrared Radiometer \& UltravioletVisible Spectrometer. The scientific objectives of the lander are: a) perform a chemical analysis of the surface materials and study the elemental composition of the surface, including radiogenic elements; b) study the physical and chemical interactions between the surface and the atmosphere; c) investigate the structure and chemical composition of the atmosphere down to the surface, including the abundances and isotopic ratios of trace and noble gases; d) perform a direct chemical analysis of cloud aerosols and analyse the vertical structure of clouds during the lander descent; e) characterize the geology of local landforms at different scales.

LLISSE is a US-contributed element (attached to the lander) that is built on the basis of high-temperature silicon carbide electronics and will capture and transmit periodic science measurements for a minimum of 60 Earth days. LLISSE is equipped with a set of sensors to measure the $2 \mathrm{D}$ wind speed and direction, temperature, pressure, some specific chemical species abundance, and the Solar radiance.

The total mass of the Venera-D vehicle is about $7000 \mathrm{~kg}$ (depending on the launch window) and there is still room for additional elements, among which can be an aerial platform for detailed cloud layer investigation or additional long-lived stations for meteorological and/or seismic measurements.

Cupid's Arrow is a small satellite mission, proposed by JPL to measure noble gases in the atmosphere of Venus (Fig. 4). It was selected by the NASA PSDS3 Program and will be based on the VERITAS (Venus Emissivity, Radio Science, INSAR, Topography, and Spectroscopy) mission mothership (Sotin et al. 2018). The mission concept is to send an atmospheric probe that skims Venus atmosphere below the homopause to collect samples of Venus atmosphere, that are then analysed by a miniaturised quadrupole ion trap mass 
Fig. 4 Picture of the Cupid's Arrow probe that will skim below Venus' homopause and will collect samples for analysis of its atmosphere (from Sotin et al. 2018)

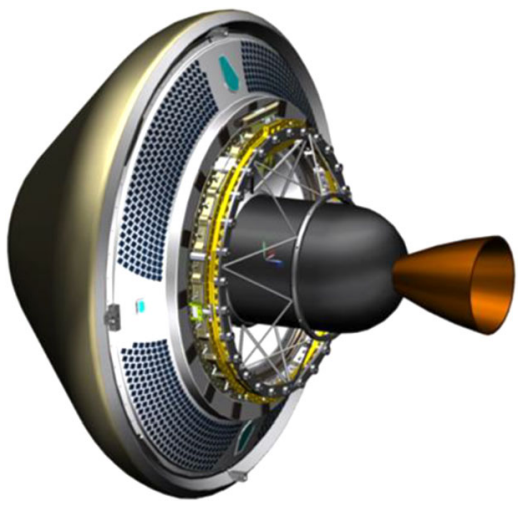

spectrometer (QITMS) once the probe is outside of Venus' atmosphere. The goal is to understand the atmospheric formation, evolution, and climate history of Venus (Chassefière et al. 2012).

\subsection{Mars}

There is compelling evidence that liquid water was abundant on early Mars, despite Mars being too cold today to sustain significant amounts of liquid water. The most likely explanation, especially in the face of the Sun having been dimmer early in its history (Girardi et al. 2000), is that early Mars had a more-effective greenhouse atmosphere. Many of the key questions about Mars over the last three decades have been centred on the nature and evolution of this early atmosphere. Measurements from spacecraft in orbit and on the surface indicate that there are insufficient carbonate deposits on the Mars surface and in the subsurface to hold enough $\mathrm{CO}_{2}$ from this early, thicker atmosphere to provide significant greenhouse warming. In that context, the Mars Atmosphere and Volatile Evolution (MAVEN) mission was designed to explore the loss of gas to space at the present epoch in detail. The goals of the mission are to understand the composition, structure, and variability of the upper-atmosphere system, the energetic drivers of the system, how they lead to loss of atmospheric gas to space, and the integrated amount of gas that has been lost to space through time (Fig. 5). The results on specific loss processes and their rates of loss are used to infer the integrated loss to space through time and the degree to which loss to space can explain the changes inferred for the Martian climate (Jakosky et al. 2015a, 2015b, 2015c; Jakosky et al. 2018 and references therein).

MAVEN has helped to determine the amount of gas lost to space through time by deriving the structure of ${ }^{38} \mathrm{Ar} /{ }^{36} \mathrm{Ar}$ between the homopause and exobase altitudes. Fractionation of argon occurs as a result of loss of gas to space by pickup-ion sputtering, which preferentially removes the lighter atom. The measurements require that $66 \%$ of the atmospheric argon has been lost to space. Thus, a large fraction of Mars' atmospheric gas has been lost to space, contributing to the transition in climate from an early, warm, wet environment to today's cold, dry atmosphere (Jakosky et al. 2017). After a full Martian year observation by MAVEN, the rate of loss of gas to space and the driving processes have been determined (Jakosky et al. 2018). Loss rates for $\mathrm{H}$ and $\mathrm{O}$ are sufficient to remove $\sim 2-3 \mathrm{~kg} / \mathrm{s}$ to space. By itself, this loss would be significant over the history of the planet. In addition, loss rates would have been greater early in history due to a more-active Sun and enhanced solar EUV flux by a factor $\sim 100-1000$ (Ribas et al. 2005; Tu et al. 2015; 
Fig. 5 Schematic showing the processes affecting the atmospheric escape on Mars. Credit: The Lunar and Planetary Institute and LASP

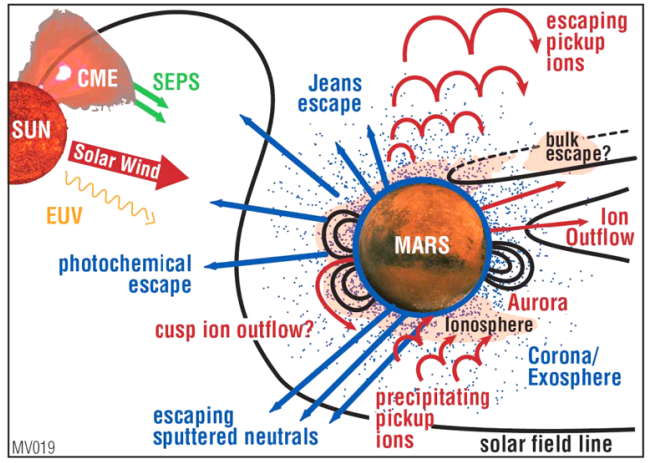

Güdel 2020), although the Sun was photometrically dimmer (Girardi et al. 2000). Integrated loss, based on current processes whose escape rates in the past are adjusted according to the expected solar evolution, would have been as much as 0.8 bar $\mathrm{CO}_{2}$ or $23 \mathrm{~m}$ global equivalent layer of $\mathrm{H}_{2} \mathrm{O}$; these losses are likely to be lower limits due to the nature of the extrapolation of loss rates to the earliest times. Combined with the lack of surface or subsurface reservoirs for $\mathrm{CO}_{2}$ that could hold remnants of an early, thick atmosphere (Edwards and Ehlmann 2015), these results suggest that loss of gas to space has been the dominant process responsible for changing the climate of Mars.

However, even if we understand the present-day $\mathrm{H}$ loss rate, it would be difficult to extrapolate to ancient times. On the one hand, loss could have been greater than it is today based on the increased solar EUV flux which would have increased the rate of photodissociation of water, and also would have provided additional heating of the upper atmosphere, both of which would increase the $\mathrm{H}$ escape rate. On the other hand, loss of $\mathrm{H}$ could have been lower in ancient times if, by having a more-Earth-like atmosphere, the Martian middle atmosphere had a more-efficient cold trap that kept the $\mathrm{H}_{2} \mathrm{O}$ from getting high in the atmosphere regardless of the dust behaviour. This uncertainty, even in the indication of the change in loss rate at earlier times, makes extrapolation difficult. Integrated loss could have been as much as an order of magnitude greater than derived from the present loss rate. As a better understanding of the relative loss rates of deuterium and $\mathrm{H}$ is obtained utilising the MAVEN IUVS observations of D (Clarke et al. 2017), a better understanding of the integrated loss of $\mathrm{D}$ through time can be expected allowing to use the observed $\mathrm{D} / \mathrm{H}$ as another way to infer the total amount of water lost through time.

Moreover, though the role of interplanetary coronal mass ejections (ICMEs) has been clearly shown (Jakosky et al. 2015b), the effects of flares and SEPs are still being analysed. In addition, the non-uniform solar wind contains what are known as stream interactive regions (SIRs), where low and high speed wind from different solar source regions collide. Observations of sun-like stars suggest that, in addition to more flares and SEPs, these events occurred more often early in the Sun's history, providing a space environment of essentially continuous ICMEs and SIRs. As these events individually result in an enhancement in loss at the present, it seems likely that they may have had similar effects early in Mars' history. There is no sufficient data yet to understand the cumulative contributions to atmospheric loss resulting from the largest events or how to extrapolate them back in time. However, because of their potential for enhancement of loss, the 0.8-bar loss described earlier is likely to be a conservative lower limit on total loss, conceivably by orders of magnitude.

Table 5 provides a summary of the major past and present missions to Mars with composition measurements. 
Table 5 Major past and present Mars missions with composition measurements

\begin{tabular}{|c|c|c|}
\hline Mission & Mission type & Composition measurements \\
\hline $\begin{array}{l}\text { Mariner 6, } 7 \\
(1969)\end{array}$ & $\begin{array}{l}\text { Interplanetary probes }+ \\
\text { Mars flybys }\end{array}$ & UV spectrometer \& IR spectrometer \\
\hline $\begin{array}{l}\text { Mars 2, } 3 \\
(1971-1972)\end{array}$ & Mars orbiter + lander & Lyman- $\alpha \mathrm{H} / \mathrm{D}$ spectrometer \\
\hline $\begin{array}{l}\text { Mariner } 9 \\
(1971-1972)\end{array}$ & Mars orbiter & UV spectrometer \& IR spectrometer \\
\hline $\begin{array}{l}\text { Viking } 1,2 \\
(1976-1982)\end{array}$ & Mars orbiter + lander & $\begin{array}{l}\text { IR spectrometer \& } \\
\text { Gas chromatograph - mass spectrometer }\end{array}$ \\
\hline $\begin{array}{l}\text { Mars Global Surveyor } \\
(1997-2006)\end{array}$ & Mars orbiter & Thermal emission imaging spectrometer \\
\hline $\begin{array}{l}\text { Mars Pathfinder (Sojourner) } \\
\text { (1997) }\end{array}$ & Mars lander + rover & $\alpha$ proton $\mathrm{X}$-ray spectrometer \\
\hline $\begin{array}{l}\text { 2001 Mars Odyssey } \\
(2001-)\end{array}$ & Mars orbiter & $\begin{array}{l}\gamma \text {-ray spectrometer \& } \\
\text { Thermal emission imaging system }\end{array}$ \\
\hline $\begin{array}{l}\text { Mars Express } \\
(2003-)\end{array}$ & Mars orbiter & $\begin{array}{l}\text { Analyser of plasma and ENAs \& } \\
\text { IR spectrometer \& Visible and IR mineralogical } \\
\text { mapping spectrometer \& } \\
\text { Sub-surface sounding radar altimeter }\end{array}$ \\
\hline $\begin{array}{l}\text { Spirit } \\
(2004-2010)\end{array}$ & Rover & $\begin{array}{l}\alpha \text {-particle X-ray spectrometer \& } \\
\text { Mössbauer spectrometer \& } \\
\text { Thermal emission spectrometer }\end{array}$ \\
\hline $\begin{array}{l}\text { Opportunity } \\
(2004-2018)\end{array}$ & Rover & $\begin{array}{l}\alpha \text {-particle X-ray spectrometer \& } \\
\text { Mössbauer spectrometer \& } \\
\text { Thermal emission spectrometer }\end{array}$ \\
\hline $\begin{array}{l}\text { Mars Reconnaissance } \\
\text { Orbiter (2006- ) }\end{array}$ & Mars orbiter & Visible and near IR spectrometer \\
\hline $\begin{array}{l}\text { Phoenix } \\
(2008)\end{array}$ & Mars lander & $\begin{array}{l}\text { Thermal and evolved gas analyser } \& \\
\text { Wet chemistry laboratory }\end{array}$ \\
\hline $\begin{array}{l}\text { Mars Science Laboratory } \\
\text { (Curiosity) } \\
(2012-)\end{array}$ & Rover & $\begin{array}{l}\text { Chemistry and camera complex (ChemCam) \& } \\
\alpha \text {-particle X-ray spectrometer \& } \\
\text { Chemistry and mineralogy X-ray powder } \\
\text { diffraction and fluorescence instrument \& } \\
\text { Sample analysis at Mars instrument \& } \\
\text { Dynamic albedo of neutrons instrument }\end{array}$ \\
\hline $\begin{array}{l}\text { Mars Orbiter Mission } \\
(2014-)\end{array}$ & Mars orbiter & $\begin{array}{l}\text { Lyman- } \alpha \text { photometer } \& \\
\text { Exospheric neutral composition analyser }\end{array}$ \\
\hline $\begin{array}{l}\text { MAVEN } \\
(2014-)\end{array}$ & Mars orbiter & $\begin{array}{l}\text { Neutral gas and ion mass spectrometer \& } \\
\text { Suprathermal and thermal ion composition } \\
\text { instrument \& Imaging UV spectrometer }\end{array}$ \\
\hline $\begin{array}{l}\text { ExoMars Trace Gas Orbiter } \\
(2016-)\end{array}$ & Mars orbiter & $\begin{array}{l}\text { IR spectrometers \& Epithermal neutron } \\
\text { detector }\end{array}$ \\
\hline $\begin{array}{l}\text { Al Amal (Hope) Emirates } \\
\text { Mars Mission } \\
\text { (launched in 2020) }\end{array}$ & Mars orbiter & UV spectrometer \& IR spectrometer \\
\hline $\begin{array}{l}\text { Tianwen-1 (Huoxing-1) } \\
\text { (launched in 2020) }\end{array}$ & $\begin{array}{l}\text { Mars orbiter }+ \text { lander }+ \\
\text { rover }\end{array}$ & $\begin{array}{l}\text { Mineralogy spectrometer } \& \\
\text { Ion and neutral particle analyser } \& \\
\text { Ground-penetrating radar }\end{array}$ \\
\hline $\begin{array}{l}\text { Mars } 2020 \text { (Perseverance) } \\
\text { (launched in 2020) }\end{array}$ & Rover & $\begin{array}{l}\text { Chemistry and camera complex (SuperCam) \& } \\
\text { X-ray fluorescence spectrometer \& } \\
\text { UV Raman spectrometer \& Ground-penetrating } \\
\text { radar }\end{array}$ \\
\hline
\end{tabular}


Since MAVEN is a single-spacecraft mission, it is not able to fully address some important plasma processes at Mars. When compared to the Earth, in the last 20 years, multispacecraft missions like Cluster, THEMIS-ARTEMIS, Van Allen Probes, and MMS have revolutionised our understanding of the causes, patterns, and variability of a wide array of space plasma phenomena in the Earth's magnetospheric environment (cf. Sect. 2.1). The ESCAPADE mission (Escape, Plasma and Acceleration Dynamics Explorers) is a twinspacecraft Mars mission that has been selected for Phase A/B study by NASA as one of three finalists in the SIMPLEX-II program (Lillis et al. 2018). It aims to similarly revolutionise our understanding of how solar wind momentum and energy flows throughout Mars' magnetosphere to drive ion and sputtering escape, two processes which have helped shape Mars' climate evolution over solar system history. ESCAPADE will measure magnetic field strength and topology, ion plasma distributions (separated into light and heavy masses), as well as suprathermal electron flows and thermal electron and ion densities from elliptical, $200 \mathrm{~km} \times \sim 7000 \mathrm{~km}$ orbits. ESCAPADE consists of two small spacecraft $(<90 \mathrm{~kg})$, traveling to Mars via solar electric propulsion as a rideshare with the Psyche metal-asteroid mission in August 2022, matching Mars' heliocentric orbit until capture and spiral-down to science orbits. ESCAPADE's strategically-designed, 1-year, 2-part scientific campaign of temporally- and spatially-separated multipoint measurements in different parts of Mars' diverse plasma environment, will for the first time unravel the cause-and-effect of solar wind control of ion and sputtering escape.

For the following years, a series of orbiters and landers is also expected to study Mars' environment and surface. NASA launched on 30 July 2020 the Mars 2020 rover, Perseverance, derived from the Curiosity rover. Its payload includes the SuperCam laser-induced breakdown spectroscopy instrument, an X-ray spectrometer, a UV spectrometer, a groundpenetrating radar and a weather station (Fig. 6). In addition to the in situ analysis, it will also collect rock samples that will then be returned to Earth in two subsequent missions, in collaboration with ESA. Mars sample return, although presenting many challenges, including the issue of planetary protection (Haltigin et al. 2018; Raulin et al. 2019; Viso 2019; Chan et al. 2020), it will allow a range of analytical techniques to be applied to them, bearing also the hope to detect possible traces of extinct or extant life (Grady 2020). Mars 2020 features also a small detachable helicopter drone, Ingenuity.

China launched on 23 July 2020 its first orbiter with lander to Mars, Tianwen-1, known also as Huoxing-1, which will deploy a small instrumented rover. The United Arab Emirates Space Agency launched on 19 July 2020 the Al Amal (Hope) orbiter to Mars, which is equipped with an Ultraviolet Spectrometer, an Infrared Spectrometer and an Exploration Imager to study the atmospheric layers of Mars and the daily and seasonal weather cycles. And a Russian carrier spacecraft will deliver the ESA ExoMars rover (expected launch in August 2022), providing surface mobility, subsurface drilling and automatic sample collection, processing, and distribution to a suite of analytical instruments dedicated to exobiology and geochemistry research.

\subsection{Mercury}

Mercury being the closest planet to the Sun, it is subject to extreme environmental conditions that led to its unique evolutionary history. It lacks the collision- dominated atmosphere of the other terrestrial planets, but has instead a tenuous surface-bounded exosphere, composed primarily of atoms and molecules released from the planet's surface (e.g. Killen and Ip 1999; Wurz and Lammer 2003; Killen et al. 2007; Vervack et al. 2010; Leblanc and Johnson 2010). Production mechanisms for Mercury's exosphere include solar wind sputtering, micrometeorite impact and photon-stimulated desorption (Wurz et al. 2010; Pfleger et al. 2015; Killen 


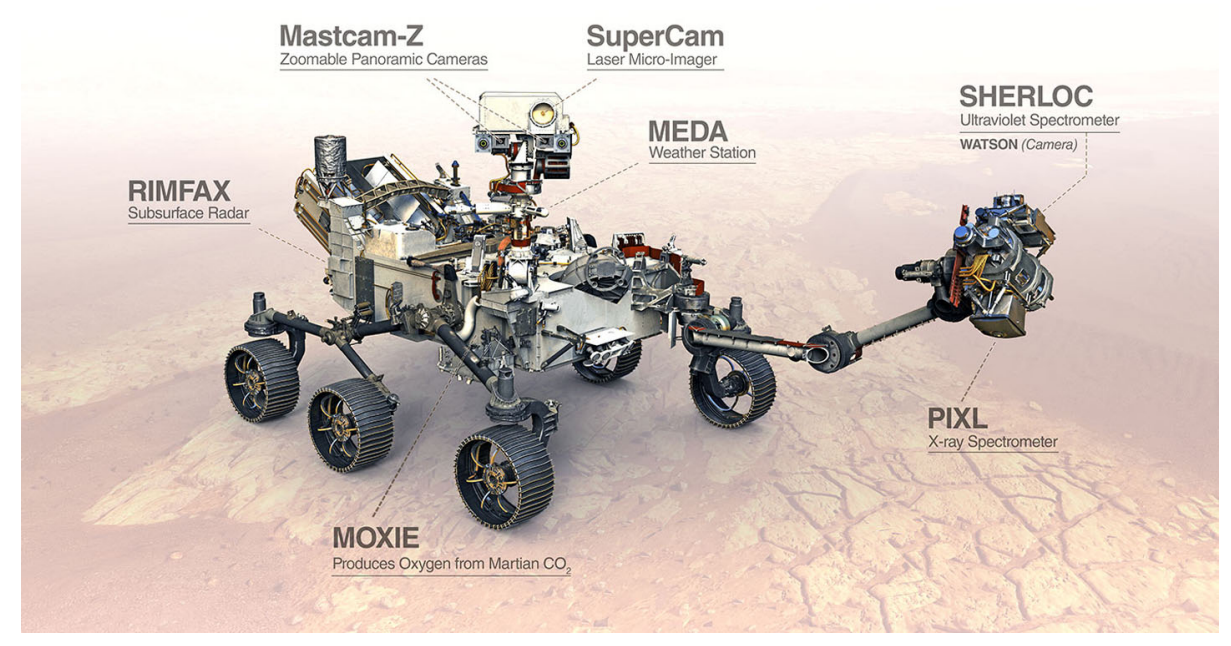

Fig. 6 Instruments onboard the Mars 2020 Perseverance rover. Credit: NASA

Table 6 Major Mercury missions with composition measurements

\begin{tabular}{lll}
\hline Mission & Mission type & Composition measurements \\
\hline $\begin{array}{l}\text { Mariner 10 } \\
(1974-1975)\end{array}$ & $\begin{array}{l}\text { Interplanetary probe }+ \\
\text { Mercury flybys }\end{array}$ & UV spectrometers \& IR radiometer \\
MESSENGER & Mercury orbiter & \\
& & $\gamma$-ray spectrometer + \\
& & X-ray spectrometer + \\
& & UV and visible spectrometer \\
& Mercury planetary & IR spectrometer \\
BepiColombo & Neutron spectrometer \\
(launched in 2018) & magnetospheric orbiter & $\gamma$-ray spectrometer + \\
& & Imaging X-ray spectrometer + \\
& UV spectrometer + \\
& & IR spectrometer + Neutron spectrometer + \\
& & Ion mass spectrometers + \\
& & Neutral mass spectrometer + \\
& & ENA imager + \\
& & Sodium atmosphere spectral imager \\
\hline
\end{tabular}

and Hahn 2015), cf. Fig. 7. The weak planetary magnetic field and the high solar wind dynamic pressure result in a highly-compressed magnetosphere and a direct interaction between the solar wind and the surface-bounded exosphere (Massetti et al. 2003, 2007; Milillo et al. 2005; Slavin et al. 2009; Raines et al. 2015; Orsini et al. 2018).

Table 6 provides a summary of the major past and present missions to Mercury with composition measurements.

The MESSENGER (MErcury Surface, Space ENvironment, GEochemistry, and Ranging) NASA mission orbited Mercury between 2011 and 2015 (Solomon et al. 2001). It provided the first comprehensive observations of Mercury's magnetosphere (Benna et al. 2009; Slavin et al. 2009; Alexeev et al. 2010; Ho et al. 2011; Zurbuchen et al. 2011), global mag- 
Fig. 7 Schematic showing sources and sinks of the Hermean exosphere (from Killen and Ip 1999)

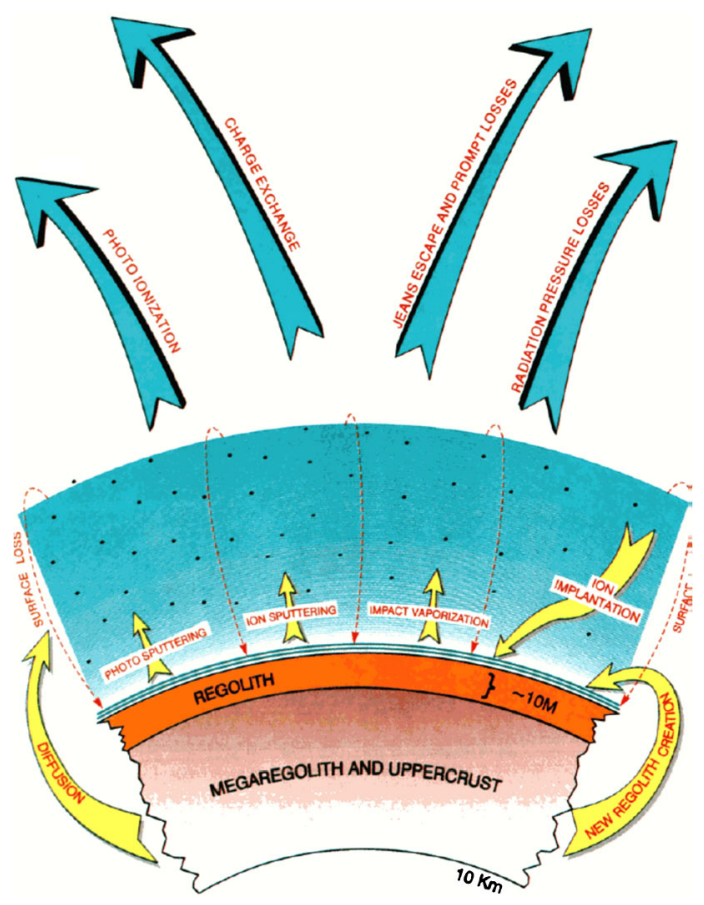

netic field (Anderson et al. 2011), exosphere (McClintock et al. 2009; Burger et al. 2010; Killen et al. 2010; Vervack et al. 2010), surface chemical composition (Denevi et al. 2009; Nittler et al. 2011) and core size and state (Genova et al. 2019).

The BepiColombo mission has been prepared jointly by ESA and JAXA (Benkhoff et al. 2010). Launched in October 2018, after a series of flybys of Earth (April 2020) and Venus (October 2020 and August 2021), it will arrive at Mercury in 2025. It is an interdisciplinary mission that comprises a Mercury Planetary Orbiter (MPO), provided by ESA, that will study the exosphere, the surface and the internal composition of the planet, and a Mercury Magnetospheric Orbiter (MMO), provided by JAXA, that will study Mercury's magnetosphere and its interaction with the solar wind. MMO is equipped with five instruments, including a Mass Spectrum Analyser (Delcourt et al. 2009) and a Mercury Sodium Atmospheric Spectral Imager (Yoshikawa et al. 2009). MPO is equipped with eleven instruments, including the SERENA (Search for Exospheric Refilling and Emitted Natural Abundances) ion and neutral atoms instrument package (Orsini et al. 2010) and the PHEBUS (Probing of Hermean Exosphere By Ultraviolet Spectroscopy) ultraviolet spectrometer to observe Mercury's exosphere (Chassefière et al. 2010). The payload of both spacecraft will provide the detailed information necessary to understand the origin and evolution of the planet itself and its surrounding environment (Milillo et al. 2020). In addition, instrumentation onboard BepiColombo will be used to test Einstein's theory of general relativity. 
Table 7 Major past and present asteroid and comet sample return missions

\begin{tabular}{ll}
\hline Mission & Sampled object \\
\hline Stardust (1999-2006) & Coma of comet Wild 2 \\
Hayabusa (2003-2010) & Asteroid 25143 Itokawa \\
Hayabusa2 (2014- ) & Asteroid 162173 Ryugu \\
OSIRIS-REx (2016- ) & Asteroid 101955 Bennu \\
\hline
\end{tabular}

\section{Asteroid Sample Return Missions}

\subsection{Asteroid Ryugu Sample Return Mission: Hayabusa2}

Hayabusa2 ("Peregrine falcon"-2) is an asteroid sample-return mission operated by JAXA. It follows on from the Hayabusa mission which in 2010 returned samples collected from the near-Earth asteroid 25143 Itokawa (Nakashima et al. 2013; Takashi et al. 2014; Fujiya et al. 2016).

Hayabusa2, launched in 2014, arrived at the near-Earth carbonaceous asteroid 162173 Ryugu in 2018. Such asteroids are assumed to have been produced from much older parent bodies through catastrophic disruption and re-accumulation of fragments during the evolution of the Solar System (O'Brien and Greenberg 2005; Sugita et al. 2019). Equipped with remote sensing instruments, sampling equipment and four small rovers, it acquired data characterising Ryugu as a spinning top-shaped rubble pile with a prominent circular equatorial ridge (Watanabe et al. 2019).

The first surface sampling by Hayabusa2 was performed in February 2019 and obtained a substantial amount of asteroid regolith material (Morota et al. 2020). The second sample collection was performed in July 2019 and used an impactor to collect sub-surface, pristine material. The sample-return capsule will re-enter Earth's atmosphere and land under a parachute in late 2020 .

\subsection{Asteroid Bennu Sample Return Mission: OSIRIS-REx}

OSIRIS-REx (Origins, Spectral Interpretation, Resource Identification, Security, Regolith Explorer) is NASA's first asteroid sample return mission (Bierhaus et al. 2018). Its goal is to obtain a sample from 101955 Bennu, a carbonaceous near-Earth asteroid, and return the sample to Earth for a detailed analysis. Bennu is a primitive body that represents the objects that may have brought prebiotic molecules and volatiles such as water to Earth (Lauretta et al. 2015).

The OSIRIS-REx spacecraft carries a suite of instruments to characterise Bennu's global properties, support the selection of a sampling site and document that site at a sub-centimetre scale. Launched in 2016, the spacecraft reached the proximity of Bennu in December 2018, where it began analysing its surface for a target sample area. Preliminary surveys of the asteroid's surface indicated Bennu to be hydrated and volatile-rich, as expected, but presenting also an unexpected surficial diversity (Lauretta et al. 2019).

In April 2020 a sample collection rehearsal was successfully performed. The touch-andgo sample collection event is now scheduled for October 2020. The sample return capsule will re-enter Earth's atmosphere in September 2023 and land under a parachute.

Table 7 provides a summary of the major past and present asteroid and comet sample return missions. 


\section{Exoplanet Missions}

\subsection{The Internal Structure and the Atmospheres of Close-in Rocky Planets}

One of the most exciting discoveries of the past decade was the detection of planets with masses smaller than $15 \mathrm{M}_{\text {Earth }}$ having densities consistent with rocky compositions. A special class of such planets are transiting ultra-short period planets (USPs) that have orbital periods of one day or less (e.g. Howard et al. 2013; Dai et al. 2017). Because of the short orbital periods, their masses and radii can be determined with a high accuracy. The reason is that the K-amplitude of the radial-velocity variation along the orbit scales with $P^{-1 / 3}$, and the radius can be determined with high accuracy because the light-curves of many transits can be averaged. Many instruments have been used to detect low-mass planets by means of Radial-Velocity (RV) measurements. Today an accuracy of $1 \mathrm{~m} / \mathrm{s}$ is achieved routinely with instruments like HARPS (Mayor et al. 2003; Bouchy et al. 2017), HARPS-N (Cosentino et al. 2012) and CARMENES (Quirrenbach et al. 2016). This accuracy allows to detect USPs with only a few $M_{\text {Earth }}$. The next step in accuracy is ESPRESSO (Pepe et al. 2010), which is designed to achieve even $0.1 \mathrm{~m} / \mathrm{s}$. ESPRESSO is also on a bigger telescope (8-m class) than the others (4-m class), which results in a significant gain in sensitivity. This allows determining the masses of USPs to a higher accuracy, and allows detecting planets of lower masses.

USPs do not have extended Hydrogen atmospheres. This is because a Hydrogen atmosphere would be quickly eroded due to the intense X-ray and extreme UV radiation from the host star (Fossati et al. 2017; Airapetian et al. 2017a; Kubyshkina et al. 2018). However, as explained below, they can have other types of atmospheres. USPs are thus ideal targets to study the internal structure and surface composition of rocky planets. USPs with masses in the range between 5 to $10 \mathrm{M}_{\text {Earth }}$ are often called "super-Earths", although it is not so obvious how Earth-like they really are. In the following, we will discuss what we have learned from transiting USPs, and how we can use them to test and constrain the various scenarios of rocky planet formation.

Table 8 gives an overview of the transiting USPs with masses of $15 \mathrm{M}_{\text {Earth }}$, or less. We also include HD 213885 b and GJ 9827 b, although their orbital periods are a little longer than one day. At least eleven of the thirteen planets are members of systems which contain up to five known planets.

The densities of the planets can be divided into two broad categories: Planets with a density consistent with an Earth-like composition (30\% metallic core, $70 \%$ silicate mantle) and planets where the density is consistent more with a Mercury-like composition (70\% metallic core, $30 \%$ silicate mantle). There is possibly even a third category composed of planets with densities 10-20\% lower than for an Earth-like composition. Examples are HD 219134 b, 55 Cancri e and WASP-47 e. As pointed out by Dorn et al. (2019), the relatively low densities of such planets can be explained with a different composition of key rocky species $(\mathrm{Fe}, \mathrm{Mg}, \mathrm{Si}, \mathrm{Ca}, \mathrm{Al}, \mathrm{Na})$. Alternatively, they could have an opaque but not very extended atmosphere. Figure 8 shows the mass-density diagram for USPs. Although the error bars for most planets are too large to put them definitely into one of the three categories, it is surprising that the majority of them fall below the line for an Earth-like composition. Perhaps most USPs belong into the third category, but the error bars of individual objects are usually too large to clearly separate them for the second category.

Up to now, the best cases for planets with a Mercury-like composition are K-106 b and $\mathrm{K} 2-229 \mathrm{~b}$. A third example is Kepler-107c, which has a density of $12.65 \pm 2.45 \mathrm{~g} \mathrm{~cm}^{-3}$ but it is not an USP (Bonomo et al. 2019). The Kepler-107-system is a remarkable one, because 


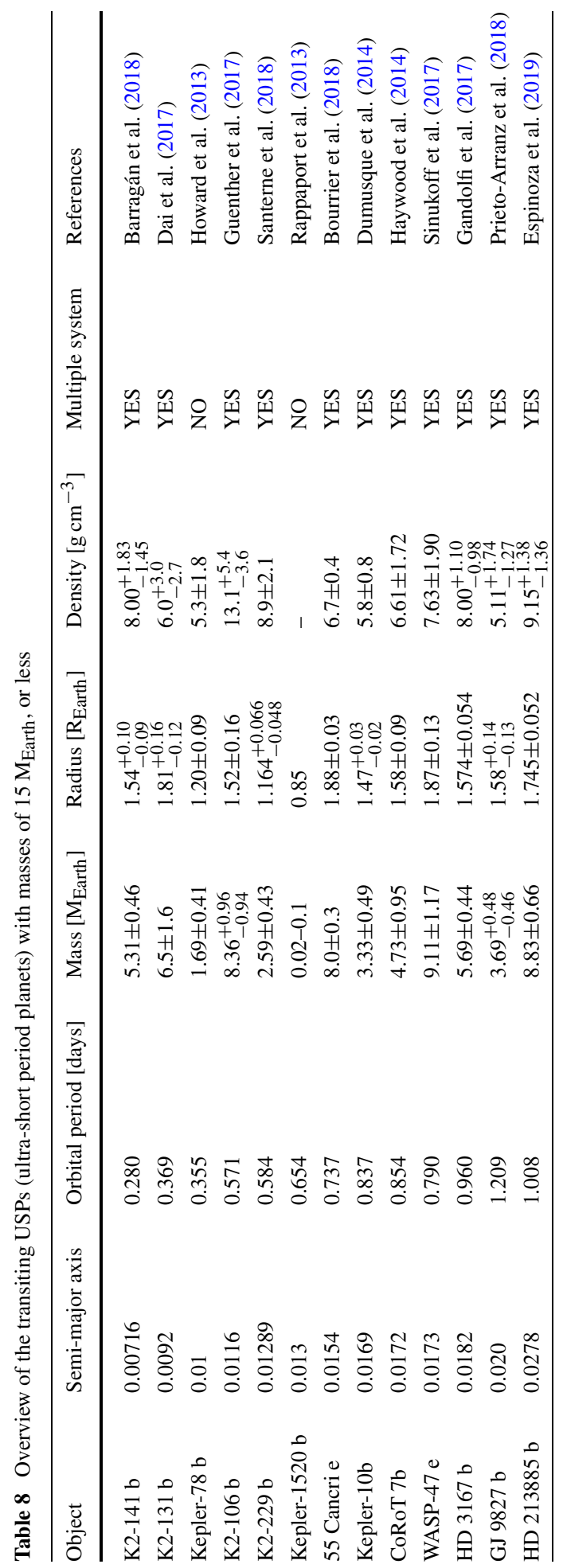


Fig. 8 Mass-density diagram of ultra-short period planets (adapted from Guenther et al. 2017 , density calculation by R. Helled)

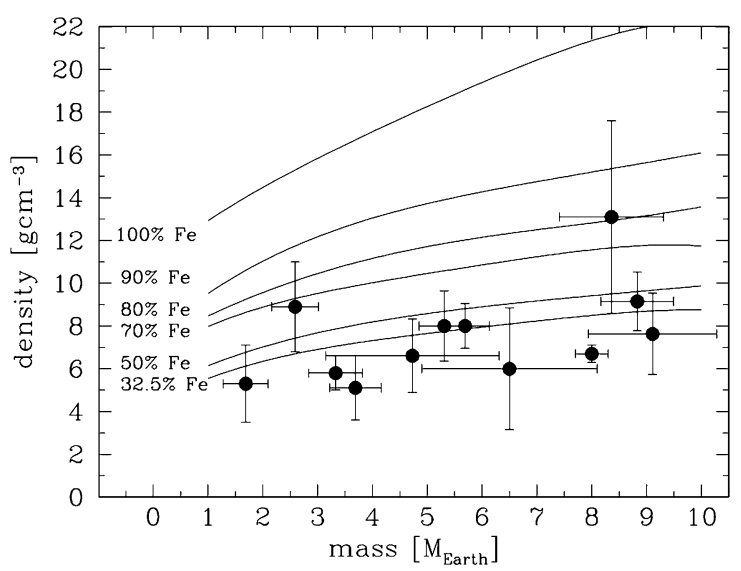

the inner planet, Kepler-107 b $(a=0.0454 \mathrm{AU})$, has a density that is consistent with an Earth-like composition, whereas Kepler-107 c ( $a=0.0606 \mathrm{AU})$ has a much higher density (Bonomo et al. 2019). Because of this unusual architecture, Bonomo et al. (2019) argue that Kepler-107 $\mathrm{c}$ is the result of a giant impact that removed the outer layers of the planet.

The densest planets, such as K2-106 b and K2-229 b, might give us important clues about the formation of planets in general. Before calling all close-in and high-density planets Mercury-like, we should keep in mind that Mercury has a semi-major axis of 0.387 AU, a radius of $0.3829 \mathrm{R}_{\text {Earth }}$, a mass of $0.055 \mathrm{M}_{\text {Earth }}$, and a density of $5.427 \mathrm{~g} \mathrm{~cm}^{-3}$. The USPs, instead, have a 50 to 170 times larger mass and they are 14 to 55 times closer to their host star than Mercury is. Such planets are not Mercury-like but far more extreme cases! It would be very interesting to know how the high-density USPs are formed. There are two possibilities: They are either the result of a giant impact (Leinhardt and Stewart 2012), or they are formed from metal rich material (Néri et al. 2019). The giant impact scenario requires a really dramatic event. Let us take K2-106 b and K2-229 b as examples. If these planets were the result of a giant impact they would have lost 40-50\% of their masses, corresponding to 20$30 \mathrm{M}_{\text {Earth }}$. According to Valencia et al. (2019) such extreme events are rare. Thus, the more high-density planets are found, the less likely this giant impact hypothesis becomes.

Planets forming close to their stars are expected to have a higher metal content. The reasons are that planetesimals in the inner region of protoplanetary disks would preferentially be iron-rich, because the condensation temperatures of metals are higher than those of silicates (Wurm et al. 2013), and because the planetesimal formation is sensitive to the size of the largest aggregates, which have a larger iron content (Kruss and Wurm 2018). Thus, if USPs form close to the host stars, we expect them to be metal rich. This scenario explains thus the formation of high density USPs and also the iron gradient in the solar system.

Generally solid planets must have different compositions, because of the different condensation temperatures, chemical composition of the disks, interior dynamics of the planets, and outgasing histories. Not only the high-density planets can be explained with these mechanisms but also the low-density ones like 55 Cancri e, WASP-47 e, and HD219134 b (Elkins-Tanton 2008; Dorn et al. 2019).

Even better than the density measurements, it would be if we could determine the composition of the planets, at least the crust, directly. This would be important not only for the high-density planets but also for all solid planets. While USPs cannot have extended Hydrogen atmospheres (Fossati et al. 2017), they can have other types of atmospheres. In the 
following we will discuss volcanic atmospheres, mineral atmospheres, which very hot planets could have, and Mercury-like atmospheres. The chemical analysis of atmospheres that contain material released from the crust would be ideal to constrain the composition of the crust.

The currently preferred object for atmospheric studies of low-mass USPs is transiting planet 55 Cancri e. It is a small planet that orbits a nearby $(d=12.590 \pm 0.012 \mathrm{pc}$; Gaia Collaboration 2018), relatively bright ( $V=5.95$; SIMBAD) star. The mass and the radius of the planet can thus be determined with very high accuracy. Bourrier et al. (2018) and Crida et al. (2018) argue that, because of the relatively low density, 55 Cancri e has an atmosphere that is $0.08+/-0.05 \mathrm{R}_{\text {planet }}$ thick. However, Madhusudhan et al. (2012) argue that there is no need for an atmosphere if it is assumed that the interior of the planet is carbonrich, which is plausible given the carbon-rich nature of the host star. As discussed above, the density distribution of USPs does not scatter around the line for planets with Earth-like compositions, as there are many planets of smaller density. Perhaps this is a sign that all these planets host atmospheres that are not extended, perhaps containing molecules with a high mean molecular mass. In the case of 55 Cancri e, there is direct evidence for the presence of an atmosphere. For example, the infrared thermal emission phase curve has a pronounced eastward-asymmetry. The hot spot thus is not at the sub-stellar point but $41 \pm 12$ degrees east of it (Demory et al. 2016a). According to Angelo and Hu (2017), this requires an atmosphere with a pressure of about 1.4 bar. Repeated infrared observations have shown that the depth of the secondary eclipse varies with time, which has also been used as an argument in favour of an atmosphere (Deming et al. 2015; Demory et al. 2016b; Tamburo et al. 2018). The idea is that the substellar hemisphere is covered by material that has a high reflectivity, which varies in size. This material could be some kind of bright clouds, or haze, in an atmosphere. The most direct evidence for the presence of an atmosphere comes from the optical transmission spectroscopy. Combining the data from five transits, Ridden-Harper et al. (2016) presented hints of $\mathrm{Na}$ and the $\mathrm{Ca}^{+}$lines originating in the atmosphere of the planet. If confirmed, this signal would correspond to an optically thick $\mathrm{Ca}^{+}$atmosphere that is five times larger than the Roche lobe. In summary, there is some evidence that 55 Cancri e has an atmosphere, hence it is possible for USPs to host some sort of atmosphere.

Since a Hydrogen-rich atmosphere is most likely ruled out, what could it be? One possibility is an atmosphere of volcanic origin. Shortly after the discovery of CoRoT-7b (Léger et al. 2009), a number of authors came up with the idea of a lava ocean at the substellar point (Briot and Schneider 2010; Barnes et al. 2010; Rouan et al. 2011). The reason that such a planet should have a lava ocean is not only the intense radiation by the host star, but also tidal heating. If the host star is strongly magnetic and the planet along its orbit crosses the stellar magnetic field lines, the sub-surface regions of USPs can be subject to induction heating (Kislyakova et al. 2017, 2018) which can, in principle, be even stronger than the tidal heating. Since all stars with outer convection zones have magnetic fields, induction heating might be very common, particularly at young ages when stellar magnetic fields are stronger.

A lava-lake model would also explain the large albedo of Kepler-10b. Lava on Earth is dark, but it is expected to be as white as snow if the temperature is much higher. The reason is that the oxides with the highest fusion temperatures are $\mathrm{MgO}(3105 \mathrm{~K}), \mathrm{CaO}(3200 \mathrm{~K})$, $\mathrm{UO}_{2}(3140 \mathrm{~K})$, and particularly $\mathrm{ThO}_{2}(3660 \mathrm{~K})$. Thus, at the highest temperatures, a lava lake will contain $\mathrm{ThO}_{2}$ particles, dispersed in the $\mathrm{Al}_{2} \mathrm{O}_{3}-\mathrm{CaO}$, and this material is white (Rouan et al. 2011). Kite et al. (2016) found that the surface composition of a magma ocean tracks bulk composition of the planet if the temperature is less than $2400 \mathrm{~K}$, but at higher temperatures the composition of the surface of the magma ocean can differ from the bulk of the ocean. Nevertheless, it would be very important to detect the material released from 
Fig. 9 Predicted dayside-averaged emission spectra of a hot rocky planet of 2 $\mathrm{R}_{\text {Earth }}$ in secondary eclipse, which has a mineral atmosphere in equilibrium with an underlying magma ocean (Ito et al. 2015)

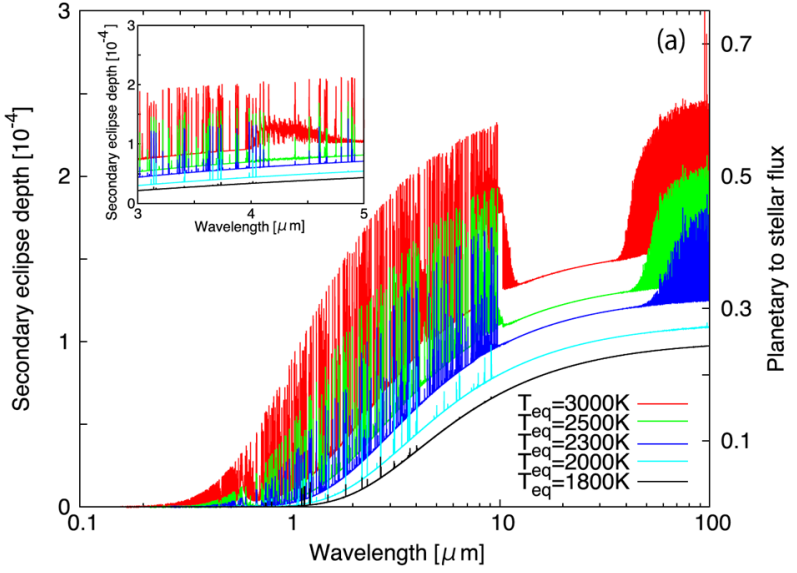

a lava ocean to constrain the chemical composition of the crust. Volcanically active planets are expected to eject mostly $\mathrm{SO}_{2}$, which would then dissociate into oxygen and sulphur atoms (Kislyakova et al. 2018). Planets with a high surface pressure are expected to have an atmosphere consisting of $\mathrm{N}_{2}$-and $\mathrm{CO}_{2}$-rich and dry volcanic gases, while low pressure ones will contain mostly sulphur-rich gases (Gaillard and Scaillet 2014). Possible tracers for such an atmosphere are the same lines that have been detected in the plasma torus of Jupiters moon Io. These are the [S III] $3722 \AA$, [O II] $3726 \AA$, [O II] $3729 \AA$, [S II] 4069 $\AA$, [S II] $4076 \AA$, [O III] $5008 \AA$, [O I] $6300 \AA$, [S III] $6312 \AA$, [S II] $6716 \AA$, [S II] 6731 $\AA$ lines, as well as the Na D-lines (Brown and Chaffee 1974; Brown et al. 1975; Brown 1976; Brown and Shemansky 1982; Morgan and Pilcher 1982; Thomas 1993; Thomas 1996; Küppers and Jockers 1995, 1997; (Kupo et al. 1976)). Magma oceans are not only present in USPs but it is generally assumed that most, if not all, rocky planets had a magma ocean at young age. The outgassing of these oceans most likely leads to $\mathrm{CO}_{2}$-rich atmospheres (Elkins-Tanton and Seager 2008; Lammer et al. 2018).

Very hot planets can have mineral atmospheres (Schaefer and Fegley 2009). The most abundant molecule, according to Miguel (2019), is expected to be $\mathrm{N}_{2}$ followed by $\mathrm{H}_{2}$ and $\mathrm{CO}$. However, transmission spectra would be dominated by $\mathrm{NH}_{3}$ and $\mathrm{HCN}$, and $\mathrm{CO}$ and HCN. According to Ito et al. (2015) a planet with a rocky molten surface should have an atmosphere that is composed of gas-phase species containing $\mathrm{Na}, \mathrm{K}, \mathrm{Fe}, \mathrm{Si}, \mathrm{SiO}, \mathrm{O}$, and $\mathrm{O}_{2}$. Figure 9 shows the predicted spectra for such planets at different temperatures (Ito et al. 2015).

Hot close-in planets could also have Mercury-like atmospheres (Vidotto et al. 2018). Mercury has an extremely thin atmosphere $\left(\sim 5 \times 10^{-12}\right.$ mbar $)$ containing $\mathrm{Na}, \mathrm{K}$ and $\mathrm{Ca}$, and other elements that are released from the crust through sputtering (Pfleger et al. 2015). Strictly speaking, this atmosphere is Mercury's exosphere (cf. also Sect. 3.3). The atmosphere of Mercury has already been detected 20 years ago using transit observations (e.g. Bida et al. 2000; Schleicher et al. 2004). Given the proximity of the USPs to their host stars, it is very well possible that they host a Mercury-like atmosphere. Since these planets are much bigger than Mercury and closer to their host stars, their atmospheres should be much thicker. For example, the surface area of CoRoT-7b is 17 times larger than Mercury and CoRoT-7b receives 250-370 times the amount of radiation. Mura et al. (2011) developed a model for Mercury-like atmosphere of CoRoT-7b. This model shows that the atmospheric loss rates of CoRoT- $7 \mathrm{~b}$ are expected to be 2-3 orders of magnitude higher than that of Mercury, forming an ionised tail of escaping particles. This tail should be observable in the 
$\mathrm{Na}$ and $\mathrm{Ca}$ resonance lines in the optical band. A first attempt to detect the atmosphere of CoRoT-7b was carried out by Guenther et al. (2011) using spectra taken with UVES at the VLT. The observations could just provide an upper limit to CoRoT-7b's atmospheric column density, indicating the need of instruments with higher sensitivity, or similar objects that orbit closer and brighter stars. Guenther et al. (2011) selected CoRoT-7 b as a target because it was the only transiting USP known at the time (Léger et al. 2009).

Luckily we now know a number of USPs orbiting stars that are closer and brighter than CoRoT-7. We mentioned already 55 Cancri. Another interesting target is HD 3167, which is 13 times brighter than CoRoT-7. We recently observed one transit of HD $3167 \mathrm{~b}$ with UVES obtaining upper limits for the emission line-fluxes relative to the star of $(0.01 \pm 1.04) \times 10^{-3}$ and $(1.02 \pm 0.96) \times 10^{-3}$ for $\mathrm{Na}$ and $\mathrm{Ca}^{+}$, respectively. For comparison, Ridden-Harper et al. (2016) detected the $\mathrm{Na}$ and $\mathrm{Ca}^{+}$at a level of $\sim 2.3 \times 10^{-3}$ and $\sim 7 \times 10^{-2}$ respectively, relative to the stellar spectrum in 55 Cancri e. However, the $\mathrm{Ca}^{+}$signal was only seen in one of the five observed transits. The difference between the two is that 55 Cancri e orbits a bit closer to its host star and it is a bit bigger than HD 3167 b. Perhaps HD 3167 b has weaker lines, or has a different crustal composition, or the absorption signal is time variable. Another interesting object is Kepler-1520 b, because it has a dusty tail containing material that is escaping from the planet (Schlawin et al. 2018). More observations of more objects are certainly required.

Although WASP-12 b is a hot Jupiter that orbits a main sequence G0 star at a distance of $0.0234 \mathrm{AU}$, it may also be interesting in the context of solid planets. The reason is that the host star, WASP-12, lacks emission components of the $\mathrm{Mg}$ II H and $\mathrm{K}$ and the $\mathrm{Ca}$ II H and $\mathrm{K}$ lines, though they are present in all stars with similar rotational velocity and spectra type. A possible explanation is that the star produces the line core emission but it is absorbed by a translucent torus of ionised $\mathrm{Mg}$ and Ca surrounding the system (Haswell et al. 2012; Fossati et al. 2013). The material composing the torus may originate from the escaping atmosphere of the hot Jupiter (Debrecht et al. 2018). However, Kislyakova et al. (2016) developed a model in which this material is released from the lava oceans of Trojan satellites of the planet. Trojan satellites of WASP-12b have not been found yet but Hippke and Angerhausen (2015) found statistical evidence for Trojans of Jupiter-like planets. After averaging all light-curves of all planets in the 50-350 days period range they found that there is a statistically significant dip corresponding to Trojans with radii between 400 and $1200 \mathrm{~km}$. For comparison, the total transiting area equivalent of small Jupiter Trojans corresponds to one body of $\sim 600 \mathrm{~km}$ radius, and the largest 32 account for an additional radius equivalent of $\sim 300 \mathrm{~km}$ (Hippke and Angerhausen 2015; Fernández et al. 2003). There is thus some statistical evidence that at least longer period planets have Trojan satellites similar to Jupiter. Given that WASP-12b orbits at 0.023 AU (Hebb et al. 2009), Trojans would have a lava ocean, if they exist.

\subsection{Exoplanet Missions}

Table 9 provides a summary of the major past and present exoplanet space missions.

The new and upcoming exoplanet missions will dramatically change that situation of known exoplanets.

The TESS (Transiting Exoplanet Survey Satellite) mission of NASA is a big leap forward in exoplanet research because it will survey the whole sky to find planets around relatively bright stars (Ricker et al. 2015). Its main targets are 200000 F5 to M5 stars (1.3 and 0.2 $\mathrm{M}_{\text {Sun }}$ ) of $m_{I}=8-12$ mag. TESS targets are thus about 100 times brighter than Kepler 
Table 9 Major past and present exoplanet space missions

\begin{tabular}{ll}
\hline Mission & Mission type \\
\hline $\begin{array}{l}\text { CoRoT (Convection, Rotation et Transits } \\
\text { planétaires) } \\
(2006-2013)\end{array}$ & Space telescope: transit method \\
$\begin{array}{l}\text { Kepler } \\
(2009-2018)\end{array}$ & Space telescope: transit method \\
$\begin{array}{l}\text { Gaia } \\
(2013-)\end{array}$ & Astrometric space observatory \\
$\begin{array}{l}\text { TESS (Transiting Exoplanet Survey Satellite) } \\
(2018-)\end{array}$ & Space telescope: transit method \\
CHEOPS (CHaracterising ExOPlanets Satellite) & \\
$(2019-)$ & Space telescope: transit method
\end{tabular}

targets. Detected planetary candidates can thus be followed-up with small- and mediumssized ground-based telescopes. The satellite has been launched in April 2018. It was initially a two-year mission but it has already been extended for another two years. Further extensions are possible. It is estimated that TESS will detect about 49 Earth-size planets $\left(R_{P}<1.25 R_{\text {Earth }}\right), 385$ super-Earths ( $\left.1.25 \mathrm{R}_{\text {Earth }}<R_{P}<2 R_{\text {Earth }}\right), 2049$ sub-Neptunes (2.0 $\mathrm{R}_{\text {Earth }}<R_{P}<4 R_{\text {Earth }}$ ), and 2529 gas-giants (Sullivan et al. 2015; Bouma et al. 2017). TESS is an all-sky survey but only the areas located within $12^{\circ}$ of the ecliptic poles are observed for 351 days. The area of the ecliptic pole fields are $905 \mathrm{deg}^{2}$, or only $2.2 \%$ of the sky. The rest of the sky is observed for a shorter time, mostly only for 27 days. Planets of small sizes and planets with long orbital periods will thus be found primarily in the ecliptic poles fields. Gas-giants with short orbital periods will be found in the whole sky.

Another new mission is ESA's CHEOPS (CHaracterising ExOPlanet Satellite) (Fortier et al. 2014; CHEOPS study team, 2013), launched in December 2019. CHEOPS is the first mission dedicated to searching for exoplanetary transits by performing ultra-high precision photometry on bright stars already known to host planets. It will thus determine the radii of planets found by other missions, for example those found by TESS. CHEOPS has a thermally stabilized f/8, Ritchey-Chrétien telescope with a diameter of $32 \mathrm{~cm}$. It is thus much larger than TESS, which has cameras of only $10 \mathrm{~cm}$ aperture. CHEOPS can thus determine the radii of planets to a much higher accuracy and can also detect smaller ones than TESS. Combined with RV-measurements, the bulk densities can be determined. It is estimated that CHEOPS will be able to detect Earth-size planets transiting G5 dwarf stars (stellar radius of $0.9 \mathrm{R}_{\odot}$ ) with $\mathrm{V}$-band magnitudes in the range $6 \leq m_{V} \leq 9$ mag. It will thus provide a major improvement in the determination of planetary radii, with a strong impact on a better determination of their possible bulk composition.

The next step is ESA's PLATO mission (PLAnetary Transits and Oscillations of stars) (Rauer et al. 2014), which is planned to be launched in 2026. The payload consists of 24 'normal' cameras. These cameras will be read out with a cadence of $25 \mathrm{~s}$ and will monitor stars with $m_{V}>8$. There are two additional cameras, which have a cadence $2.5 \mathrm{~s}$. These fast cameras will be used for stars with $m_{V} \sim 4$ to 8 . The 'normal' cameras are arranged in four groups of six. Each of them has a field-of view of $1100 \mathrm{deg}^{2}$. The central field were all cameras overlap has a size of $301 \mathrm{deg}^{2}$, and the whole field is $2250 \mathrm{deg}^{2}$. The core of the PLATO mission targets are 22000 FGK-stars with $m_{v}<11$ mag. The light-curves of these PLATO will have a noise level of $34 \mathrm{ppm} / \mathrm{h}$. For comparison, the transit depth of 2(1) $\mathrm{R}_{\text {Earth }}$ 
planet orbiting solar-like star is 334(84) ppm. Simulations show that PLATO will be capable of discovering 4000 super-Earth planets around stars with $m_{v}<11$ mag. Because PLATO will stare at one field for at least two years, it will be possible to detect Earth-sized planets of long orbital period, e.g. planets in the habitable zone (HZ). It is expected that PLATO will find 40-70 super-Earths in the HZ of G-type stars. For detecting planets with periods of $90 \mathrm{~d}<P<500 \mathrm{~d}$ PLATO outperforms Kepler by a factor 10, and TESS by a factor 1000 . PLATO will provide a huge increase in the number of small transiting planets, and many of them will be USPs.

Detailed studies of USPs will be possible with ESA's ARIEL (Atmospheric Remotesensing Infrared Exoplanet Large-survey) mission (Puig et al. 2018; Tinetti et al. 2016, 2018; Ludovic 2018), planned to be launched in 2028. Many exoplanets will certainly be observed with the future James Webb Space Telescope (JWST) of NASA, but ARIEL will be the first dedicated space mission for atmospheric studies. It will measure the chemical composition and thermal structure of a large number $(\sim 1000)$ of exoplanets that have temperatures of at least $600 \mathrm{~K}$. ARIEL will thus provide a truly representative picture of the chemical nature of exoplanets, including gas giants, Neptunes, super-Earths and Earth-size planets around a range of host star types using transit spectroscopy. In this way it will answer the following questions: what exoplanets are made of and how they form and have evolved. ARIEL has a three-mirror off-axis telescope with an elliptical primary of $1.1 \times 0.7 \mathrm{~m}$. The VIS channel has three photometric bands: 0.5-0.6 $\mu \mathrm{m}, 0.6-0.81 \mu \mathrm{m}$, and 0.81-0.1. $\mu \mathrm{m}$. The NIR spectrographs cover the wavelength bands $1.1-1.95 \mu \mathrm{m}(R=20), 1.95-3.9 \mu \mathrm{m}(R=100)$, and 3.9-7.8 $\mu \mathrm{m}(R=30)$. These wavelength ranges contain all the expected major atmospheric gases from e.g. $\mathrm{H}_{2} \mathrm{O}, \mathrm{CO}_{2}, \mathrm{CH}_{4}, \mathrm{NH}_{3}, \mathrm{HCN}, \mathrm{H}_{2} \mathrm{~S}$ through to the more exotic metallic compounds, such as $\mathrm{TiO}, \mathrm{VO}$, and condensed species.

The next generation of 30 and 40 m-class ground-based telescopes, for example the ELT (Extremely Large Telescope), for which the first light is planned for 2025, will also allow us to study the atmospheres in detail (Rodler 2018). They will even allow a much higher spectral resolution than it will be possible with ARIEL. Possibly the most interesting results can be obtained when the high-resolution spectra, obtained with ground-based telescopes, will be combined with spectra obtained with ARIEL.

\section{3 $\eta$-Earth and the Relevance of $\mathrm{N}_{2}-\mathrm{O}_{2}$ Dominated Atmosphere Detection by Future Space Missions}

Since the past two decades, the number of exoplanets continues to grow and an understanding of the frequency of habitable Earth-like exoplanets remains a major area of interest. The search for life beyond Earth and the Solar System focuses on finding rocky exoplanets orbiting a star at the orbit location within the so-called habitable zone that allows for the existence of liquid water (e.g. Hart 1979; Lammer et al. 2009; Kasting 2010; Wolf 2017). The mean number of rocky exoplanets per star that reside within their respective habitable zone is called eta-Earth, or $\eta E$. Planets are thought to be rocky with radii less than $1.6 \mathrm{R}_{\text {Earth }}$ (Rogers 2015). Usually, $\eta E$ is calculated separately for each stellar spectral type (Kasting et al. 1993; Kopparapu et al. 2013).

NASA's Kepler space telescope explored the parameter-space of Earth-sized exoplanets. One of the mission goals was to establish an estimate for $\eta E$. However, a large number of possible $\eta E$-values lying in the wide range between $0.01-0.35$ were estimated by various researchers (Catanzarite and Shao 2011; Petigura et al. 2013; Silburt et al. 2015; Traub 2016; Barbato et al. 2018; Zink and Hansen 2019). A main reason for these significant differences in $\eta E$ values is that the climate models differ concerning the precise location annulus of 
these terrestrial planets that could potentially have liquid water on the surface, the maximum radius of a planet being "rocky" is still debated, and the lifetime of the Kepler mission was not enough to have complete statistics for FGK stars.

An additional problem with this size-related $\eta E$ definition is the lack of knowledge of the potential accretion histories of these $\eta E$-like planets, their internal composition, tectonic regime, and more (see Bermingham et al. 2020; Lammer et al. 2020b). Each of the mentioned studies have chosen particular limits within possible ranges, but neglected potential relevant parameters such as the distribution and variation of radioactive heat producing elements (see O'Neill et al. 2020) within planetary building blocks and consequently within Earth-sized exoplanets. Moreover, the billion years lasting evolution of planetary atmospheres (see Avice and Marty 2020; Lammer et al. 2020a), including their shaping factors related to their host stars activity evolution (see Güdel 2020), are also neglected in such estimates. $\eta E$ is currently the next parameter in the Drake equation where we can get a robust estimate, that is $N_{\text {civ }}=R \times f_{p} \times \eta E \times f_{1} \times f_{\mathrm{i}} \times f_{\mathrm{c}} \times L$, which gives the number $\left(N_{\text {civ }}\right)$ of "hypothetical" civilizations in the Galaxy that are able to communicate through electromagnetic waves (e.g. Sagan 1963; Carigi 2015). The other values in the Drake equation are $R$, the average rate of star formation in the galaxy, $f_{p}$, the fraction of stars that have planets, $f_{l}$, the fraction of these planets that could support and actually develop life at some point, $f_{i}$, the fraction of these planets with intelligent life, $f_{c}$, the fraction of civilizations that release detectable signs of their existence into space, and $L$, the timespan over which such civilizations actually release these signals into space. These last three parameters have the largest uncertainty and have no scientific estimate at this time.

Sara Seager (2017) proposed a revised Drake equation that aims towards biosignature gas detection in exoplanetary atmospheres. This equation can be written as $N_{\text {bio }}=N_{\text {star }}$ $\times f_{\mathrm{q}} \times f_{\mathrm{HZ}} \times f_{\mathrm{o}} \times f_{\text {live }} \times f_{\mathrm{s}}$, where $N_{\text {bio }}$ is the number of planets with detectable signs of life, $N_{\text {star }}$, the number of stars observed, $f_{\mathrm{q}}$, the fraction of stars that are quiet enough for terrestrial planets to sustain secondary atmospheres, $f_{\mathrm{HZ}}$, the fraction of stars with rocky planets in the habitable zone, $f_{\mathrm{o}}$, the fraction of these planets that can be observed, $f_{\text {live }}$, the fraction of these planets that have life, and $f_{\mathrm{s}}$, the fraction on which life produces a detectable atmospheric biosignature.

In order to search for life and biospheres on Earth-like exoplanets, various molecular biosignatures have been studied and proposed (e.g. Lovelock 1975; Segura et al. 2003; Grenfell et al. 2007a, 2007b, 2010; Scalo et al. 2007; Cockell et al. 2009; Schwieterman et al. 2017). Besides biosignature molecules such as $\mathrm{N}_{2} \mathrm{O}$ (Sagan et al. 1993; Segura et al. 2005; Rauer et al. 2011; Rugheimer et al. 2013, 2015; Airapetian et al. 2017b), $\mathrm{CH}_{4}$ (Sagan et al. 1993; Krasnopolsky et al. 2004; Rugheimer et al. 2015; Airapetian et al. 2017b), $\mathrm{CH}_{3} \mathrm{Cl}$ (Segura et al. 2005; Rugheimer et al. 2015), $\mathrm{NH}_{3}$ (Seager et al. 2013a, 2013b), sulfur gases and $\mathrm{C}_{2} \mathrm{H}_{6}$ (Pilcher 2003; Domagal-Goldman et al. 2011), and organic hazes (Arney et al. 2016, 2017), $\mathrm{O}_{2}$ has long been recognized as a key biosignature. This is because on Earth it is only produced by life and it has a high concentration in the modern terrestrial atmosphere and has a strong spectral feature at 0.76 microns. In the IR, $\mathrm{O}_{2}$ can be detectable through its photochemical proxy, $\mathrm{O}_{3}$ (Owen 1980; Léger et al. 1993; Sagan et al. 1993; Des Marais et al. 2002; Léger et al. 2011; Airapetian et al. 2017b).

Oxygen is thought to be a necessary ingredient for the evolution of complex life forms on habitable planets due to the large redox energy gradient pair of aerobic respiration (Catling et al. 2005; Meadows et al. 2018). There are known abiotic pathways to create $\mathrm{O}_{2}, \mathrm{CH}_{4}$ and $\mathrm{N}_{2} \mathrm{O}$ (see e.g. Meadows et al. 2018; Guzmán-Marmolejo et al. 2013; Airapetian et al. 2016 and references therein). As such, no single biosignatures will confirm life by its own, 
as abiotic production scenarios under certain environmental conditions could always remain an alternative explanation for these gases.

As recently discussed by Stüeken et al. (2016) and Lammer et al. (2019), the two main atmospheric species in present-day Earth's atmosphere, $\mathrm{N}_{2}$ and $\mathrm{O}_{2}$, constitute a geobiosignature for a biosphere that is populated by denitrifying life-forms (see Stuieken et al. 2020; Lloyd et al. 2020). According to the current state of knowledge, a long-time stable $\mathrm{N}_{2}-\mathrm{O}_{2}$ atmosphere, together with a considerably lower share of $\mathrm{CO}_{2}$, seem to have strong correlations to the presence of aerobe lifeforms and even indicate an active tectonic regime (Stüeken et al. 2016; Lammer et al. 2019).

A physically untouched $\mathrm{N}_{2}$ atmosphere is chemically exceedingly stable, due to its strong triple bond (e.g. Zumdahl and Zumdahl 2010). However, a surface totally or partly covered by liquid water moistens the air, providing conditions for efficient lightning fixation which converts $\mathrm{N}_{2}$ into water soluble molecules (e.g. Chameides and Walker 1981; NavarroGonzález et al. 2001). This nitrogen is then dissolved in lakes/oceans, where it can form sediments (e.g. Voss et al. 2013). Impactor fixation (Fegley et al. 1986; Chyba and Sagan 1992; Parkos et al. 2018) and a productive photochemistry (Zahnle 1986; Tian et al. 2011; Airapetian et al. 2016) contribute to this depletion, especially on young planets. If the magnetospheric protection capability is low, cosmic rays may also play a role in nitrogen fixation (Navarro-González et al. 1998; Grenfell et al. 2012; Cooray 2015; Tabataba-Vakili et al. 2016). The strong return fluxes of nitrogen from the oceans into the atmosphere, observed on present Earth, are due to biological processes such as denitrification and anammox (e.g. Fowler et al. 2013; Zerkle and Mikhail 2017). In addition, these processes are effective especially in an oxidized environment, where nitric oxide is also a common reactant. In the later stages of a planet's evolution, it is not likely that volcanic nitrogen outgassing is strong enough to maintain more than a few mbar $\mathrm{N}_{2}$ in an atmosphere; if one disregards biological influences on present Earth, one finds a net ingassing flux of $\mathrm{N}_{2}$, since nitrogen fixation is stronger than nitrogen outgassing (Cartigny and Marty 2013; Lammer et al. 2019).

Plate tectonics remain a crucial factor for shaping a stable $\mathrm{N}_{2}-\mathrm{O}_{2}$ atmosphere. The subduction of oxidised material supports nitrogen outgassing through volatilisation of stored reduced nitrogen compounds, as well as it leads to a partly re-outgassing of sedimented nitrogen through metamorphism (Mikhail and Sverjensky 2014; Barry and Hilton 2016). Through gradually replacing oxidised surface, active tectonics help to remove $\mathrm{CO}_{2}$ from the atmosphere through weathering (Walker et al. 1981; Krissansen-Totton et al. 2018). Combined with the outcome of the previous paragraph, a purely $\mathrm{N}_{2}$-dominated atmosphere is possible only with an active tectonic regime that has led to $\mathrm{CO}_{2}$ depletion and to a geologically-induced high volcanic outgassing rate. Here, converting of water-dissolved nitrogen into $\mathrm{N}_{2}$ in volcanic environments at mid-ocean ridges, a process whose impact has not been quantified yet, could play a role (Kasting et al. 1993). Under such conditions, anaerobe life can exist undetectable with the here presented method. All in all, an $\mathrm{N}_{2}$ atmosphere constitutes a geosignature, indicating some sort of tectonic activity.

In a pure $\mathrm{N}_{2}$ atmosphere without any reaction partners (e.g. $\mathrm{CO}_{2}, \mathrm{CH}_{4}$ ), fixation is not possible; on the contrary, the more reaction partners, the more efficient lightning fixation works (Navarro-González et al. 2001). Therefore, when $\mathrm{O}_{2}$ joins as a bulk gas, either $\mathrm{N}_{2}$ or $\mathrm{O}_{2}$ is expected to be depleted in the long-term. Generally, the atmospheric presence of $\mathrm{O}_{2}$ in greater amounts needs an oxidized planet's surface and a lithosphere (e.g. Catling et al. 2005; Catling 2014). $\mathrm{O}_{2}$ is required by complex life, not only in a chemical/metabolic sense but also as $\mathrm{O}_{3}$ for shielding UV radiation (e.g. Levine 2014). Under the conditions discussed here, it can serve as indicator for complex lifeforms too. Both, the $\mathrm{O}_{2}$ as well as 
the $\mathrm{N}_{2}$ cycle, contain strong biological return fluxes to the atmosphere (e.g. Galloway 2003; Petsch 2014).

These deliberations lead to the conclusion that an $\mathrm{N}_{2}-\mathrm{O}_{2}$-atmosphere is most likely possible only under the influence of aerobic lifeforms. This makes the combination of $\mathrm{N}_{2}$ and $\mathrm{O}_{2}$ as bulk gases and a negligible amount of $\mathrm{CO}_{2}$ the most likely geo-biosignature for life as we know it (Lammer et al. 2019). If such an atmosphere is additionally populated with $\mathrm{O}_{3}$, $\mathrm{H}_{2} \mathrm{O}, \mathrm{CH}_{4}$, and $\mathrm{N}_{2} \mathrm{O}$, then the corresponding planet can decisively indicate the presence of live on an Earth-like habitat (Airapetian et al. 2017b).

If one takes Drake's equation or the modified one by Seager (2017) and introduces the challenge of the detection of $\mathrm{N}_{2}-\mathrm{O}_{2}$ dominated atmospheres at rocky planets inside habitable zones as an updated $\eta E_{\mathrm{N} 2-\mathrm{O} 2}$-constrain, then the Drake equation can be written as $N=$ $R \times f_{\mathrm{p}} \times \eta E_{\mathrm{N} 2-\mathrm{O} 2} \times f_{\mathrm{i}} \times f_{\mathrm{c}} \times L$. Seager's equation transforms then to an equation for the number of Earth-like habitats, equal here to the number of planets with an $\mathrm{N}_{2}-\mathrm{O}_{2}$-dominated atmosphere: $N_{\text {Earth }}=N_{\text {star }} \times f_{\mathrm{q}} \times f_{\mathrm{HZ}} \times \eta E_{\mathrm{N} 2-\mathrm{O} 2}$.

One reason why $\mathrm{N}_{2}$ atmospheres were not considered as an indirect biosignature in the past might be the very tricky detection of $\mathrm{N}_{2}$ (and the detection of the domination of $\mathrm{N}_{2}$ ), which is challenging even for future space telescopes. In an $\mathrm{N}_{2}-\mathrm{O}_{2}$-dominated atmosphere, $\mathrm{N}_{2} \mathrm{O}$ molecules can be also deeply linked to biological and atmospheric processes (Muller 2013). From the Earth it is known that $\mathrm{N}_{2} \mathrm{O}$ would be efficiently depleted by photodissociation in the troposphere, if $\mathrm{O}_{3}$ would not protect it. Furthermore, the strength of molecular transition bands of $\mathrm{N}_{2} \mathrm{O}$ and $\mathrm{NO}_{2}$ molecules lie in the blue optical region and at near-UV wavelengths, but the much stronger lines of $\mathrm{O}_{2}$ and $\mathrm{O}_{3}$ are located at similar wavelengths (Bétrémieux and Kaltenegger 2013), which makes the detection of $\mathrm{N}_{2} \mathrm{O}$ and $\mathrm{NO}_{2}$ challenging. In addition, $\mathrm{N}_{2} \mathrm{O}$ molecules have two absorption bands in the infrared at about 4.5 and $7.8 \mathrm{~mm}$, but they are generally rather weak (Rauer et al. 2011; Hedelt et al. 2013), though could be detectable at higher concentrations around quiescent $\mathrm{M}$ dwarfs at $17 \mathrm{mi}-$ crons (Rugheimer et al. 2015).

Infrared absorption features of $\mathrm{O}_{2} \cdot \mathrm{N}_{2}$ dimers at $1.26 \mathrm{~mm}$ should be detectable in the transmission spectrum of an Earth-like habitat, if the spectral resolution and signal-to-noise ratio are high enough (Pallé et al. 2009). Misra et al. (2014) carried out a study in which the dimers of $\mathrm{O}_{2} \cdot \mathrm{O}_{2}$ were analysed and concluded that they would be extremely valuable for the characterisation of Earth-like planets, indicating perhaps an anti-biosignature with large $\mathrm{O}_{2}$ concentrations produced by a run-away greenhouse or similar photochemical process.

Observationally, the $\mathrm{O}_{2} \cdot \mathrm{N}_{2}$ dimer could be detected and measured from the ground using the ELTs, or from space by the proposed mission HabEX (Fig. 10), which will be able to observe the reflected light of exoplanets from ultraviolet to near-infrared wavelengths directly, through blocking out of the parent's light with a coronograph and a starshade (Gaudi et al. 2018, 2019). The upper atmosphere of an Earth-like habitat would be composed mainly of $\mathrm{H}, \mathrm{N}$, and $\mathrm{O}$ atoms. In such a case, charge exchange with stellar wind ions and resonant scattering would lead to the formation of a number of emission lines for these atoms, of which the strongest ones would be located between X-rays and optical wavelengths. Due to the low atmospheric density, these atoms can then be detected in the far-UV wavelengths $(122-200 \mathrm{~nm})$ of transmission spectra from transiting Earth-like habitats.

These absorption lines might be detectable by future large-aperture multipurpose telescopes, such as LUVOIR (Dalcanton et al. 2015), due to their unprecedented high far-UV sensitivity of the LUMOS (France et al. 2017) and POLLUX (Bouret et al. 2018) spectrographs, and HabEX. The LUVOIR (Fig. 11) and HabEx (Fig. 10) mission concepts, currently studied by NASA, or the ESA mission concept LIFE (Large Interferometer for Exoplanets) (Quanz et al. 2019), open the perspective of perhaps discovering inhabited worlds orbiting stars other than our Sun. 
Fig. 10 Habitable Exoplanet Observatory HabEx (image by NASA)

Fig. 11 Large UV Optical Infrared Surveyor LUVOIR (image by NASA)
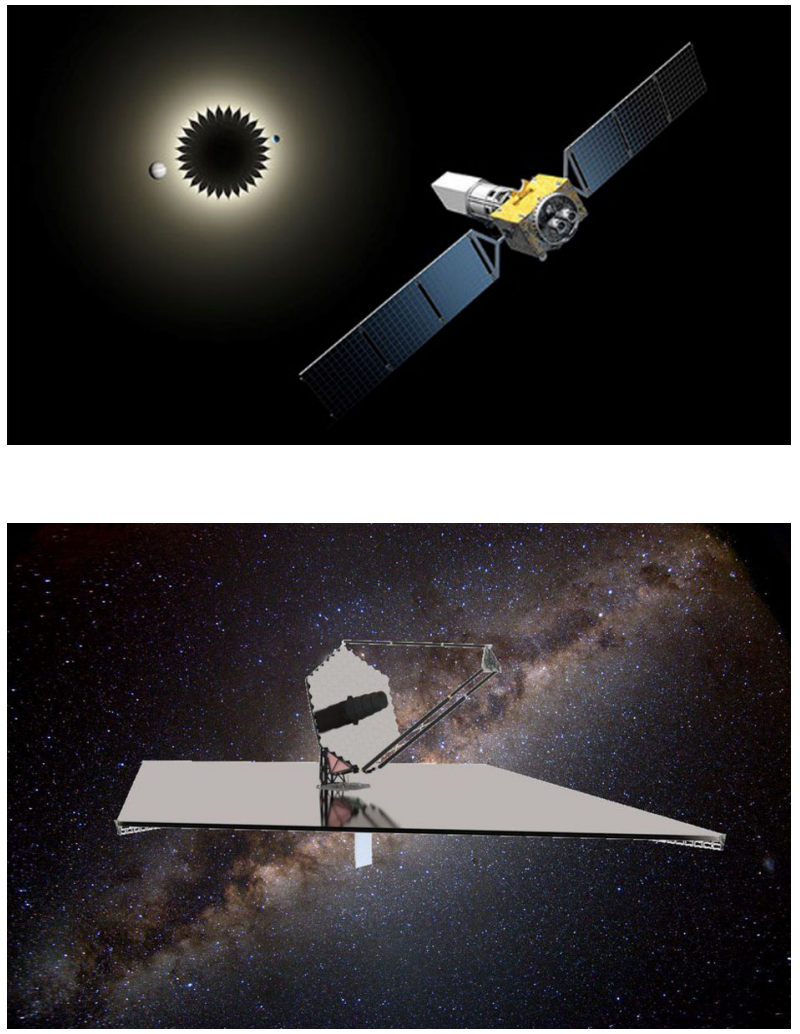

$\mathrm{N}_{2}-\mathrm{O}_{2}$ atmospheres are also transparent for starlight due to the oxidation of visible-light absorbers through $\mathrm{O}_{2}$ molecules, which leads via Rayleigh scattering on these molecules to its famous "pale blue dot" appearance (Krissansen-Totton et al. 2016). By considering this process, an Earth-like Rayleigh absorption feature in the visible should then also be consistent with an $\mathrm{N}_{2}-\mathrm{O}_{2}$ dominated atmosphere and, therefore, could be considered as an indirect biosignature from an Earth-like habitat.

Finally, if $\mathrm{N}_{2}$-dominated atmospheres are rare, due to the complex interplay of atmosphere, lithosphere, and biosphere (see O'Neill et al. 2020; Stüeken et al. 2020; Lloyd et al. 2020), then one may expect that the atmospheres of most Earth-sized exoplanets in the habitable zones would be $\mathrm{CO}_{2}$-dominated (Lammer et al. 2019). Carbon dioxide molecules present a number of absorption bands in the IR, making them detectable from space with the future James Webb Space Telescope (JWST) and ARIEL space observatories, and from the ground with high-resolution spectrographs attached to the ELTs.

JWST is a visible to infrared mission planned to be launched by NASA, in cooperation with ESA (Ariane 5 launch) and the Canadian Space Agency, in 2021. JWST will be able to characterise atmospheres of exoplanets through transmission spectroscopy and secondary eclipse measurements. JWST will be the first mission capable of characterising an Earth-like exoplanet orbiting an M dwarf. However, it is expected that such a measurement will require hundreds of hours and JWST is a community-wide telescope, therefore such an observation will be limited to only a small number of terrestrial exoplanets (Kaltenegger and Traub 2009). 
If $\mathrm{CO}_{2}$-dominated atmospheres are common on Earth-sized exoplanets within habitable zones, these telescopes will detect such atmospheres by characterizing Earth-sized exoplanets discovered by the ongoing TESS and the future PLATO missions. For the far future (2030+), however, a realistic quantification of $\eta E$ that results in a less speculative occurrence rate of Earth-like habitats, would require the detection of $\mathrm{N}_{2}-\mathrm{O}_{2}$-dominated atmospheres and hence $\eta E_{\mathrm{N} 2-\mathrm{O} 2}$, by using the next generation ground- and space-based telescopes, such as the ground-based ELTs or the space-based LUVOIR.

Acknowledgements The authors thank the International Space Science Institute in Bern, Switzerland, for its support of the Workshop on "Reading Terrestrial Planet Evolution in Isotopes and Element Measurements". I. Dandouras, M. Blanc and C. Mazelle thank CNES for its support.

Publisher's Note Springer Nature remains neutral with regard to jurisdictional claims in published maps and institutional affiliations.

Open Access This article is licensed under a Creative Commons Attribution 4.0 International License, which permits use, sharing, adaptation, distribution and reproduction in any medium or format, as long as you give appropriate credit to the original author(s) and the source, provide a link to the Creative Commons licence, and indicate if changes were made. The images or other third party material in this article are included in the article's Creative Commons licence, unless indicated otherwise in a credit line to the material. If material is not included in the article's Creative Commons licence and your intended use is not permitted by statutory regulation or exceeds the permitted use, you will need to obtain permission directly from the copyright holder. To view a copy of this licence, visit http://creativecommons.org/licenses/by/4.0/.

\section{References}

V.S. Airapetian, A. Glocer, G. Gronoff, E. Hébrard, W. Danchi, Prebiotic chemistry and atmospheric warming of early Earth by an active young Sun. Nat. Geosci. 9, 452-455 (2016). https://doi.org/10.1038/ ngeo2719

V.S. Airapetian, A. Glocer, G.V. Khazanov, R.O.P. Loyd, K. France, J. Sojka, W.C. Danchi, M.W. Liemohn, How hospitable are space weather affected habitable zones? The role of ion escape. Astrophys. J. Lett. (2017a). https://doi.org/10.3847/2041-8213/836/1/L3

V.S. Airapetian, C.H. Jackman, M. Mlynczak, W. Danchi, L. Hunt, Atmospheric beacons of life from exoplanets around G and K stars. Sci. Rep. 7, 14141 (2017b). https://doi.org/10.1038/s41598-017-14192-4

I.I. Alexeev, E.S. Belenkaya, J.A. Slavin, H. Korth, B.J. Anderson, D.N. Baker, S.A. Boardsen, C.L. Johnson, M.E. Purucker, M. Sarantos, S.C. Solomon, Icarus (2010). https://doi.org/10.1016/j.icarus.2010.01.024

B.J. Anderson, C.L. Johnson, H. Korth, M.E. Purucker, R.M. Winslow, J.A. Slavin, S.C. Solomon, R.L. McNutt Jr., J.M. Raines, T.H. Zurbuchen, The global magnetic field of Mercury from MESSENGER orbital observations. Science (2011). https://doi.org/10.1126/science.1211001

I. Angelo, R. Hu, A case for an atmosphere on super-Earth 55 Cancri e. Astron. J. 154, 232 (2017)

V. Angelopoulos, The ARTEMIS mission. Space Sci. Rev. (2011). https://doi.org/10.1007/s11214-0109687-2

G.N. Arney, S.D. Domagal-Goldman, V.S. Meadows, E.T. Wolf, E. Schwieterman, B.D. Charnay, M.W. Claire, E. Hébrard, M.G. Trainer, The pale orange dot: the spectrum and habitability of hazy Archean Earth. Astrobiology 16, 873-899 (2016). https://doi.org/10.1089/ast.2015.1422

G.N. Arney, V.S. Meadows, S.D. Domagal-Goldman, D. Deming, T.D. Robinson, G. Tovar, E.T. Wolf, E. Schwieterman, Pale orange dots: the impact of organic haze on the habitability and detectability of Earthlike exoplanets. Astrophys. J. 836, 49 (2017). https://doi.org/10.3847/1538-4357/836/1/49

G. Avice, B. Marty, Perspectives on atmospheric evolution from noble gas and nitrogen isotopes. Space Sci. Rev. 216, 36 (2020). https://doi.org/10.1007/s11214-020-00655-0

R.A. Bamford, E.P. Alves, F. Cruz, B.J. Kellett, R.A. Fonseca, L.O. Silva, R.M.G.M. Trines, J.S. Halekas, G. Kramer, E. Harnett, R.A. Cairns, R. Bingham, 3D PIC simulations of collisionless shocks at lunar magnetic anomalies and their role in forming lunar swirls. Astrophys. J. (2016). https://doi.org/ 10.3847/0004-637X/830/2/146

D. Barbato, A. Sozzetti, S. Desidera, M. Damasso, A.S. Bonomo, P. Giacobbe, L.S. Colombo, C. Lazzoni, R. Claudi, R. Gratton, G. Locurto, F. Marzari, C. Mordasini, Exploring the realm of scaled solar system analogues with HARPS. Astron. Astrophys. 615 (2018). https://doi.org/10.1051/0004-6361/201832791 
R. Barnes, S.N. Raymond, R. Greenberg et al., CoRoT-7b: super-Earth or super-Io? Astrophys. J. 709, 95 (2010)

O. Barragán, D. Gandolfi, F. Dai et al., K2-141 b. A 5-Mearth super-Earth transiting a K7 V star every 6.7 h. Astron. Astrophys. 612, A95 (2018)

P.H. Barry, D.R. Hilton, Release of subducted sedimentary nitrogen throughout Earth's mantle. Geochem. Perspect. Lett. 2, 148-159 (2016). https://doi.org/10.7185/geochemlet.1615

J. Benkhoff, J. van Casteren, H. Hayakawa, M. Fujimoto, H. Laakso, M. Novara, P. Ferri, H.R. Middleton, R. Ziethe, BepiColombo-comprehensive exploration of Mercury: mission overview and science goals. Planet. Space Sci. (2010). https://doi.org/10.1016/j.pss.2009.09.020

M. Benna et al., Modeling of the magnetosphere of Mercury at the time of the first MESSENGER flyby. Icarus (2009). https://doi.org/10.1016/j.icarus.2009.11.036

M. Benna, P.R. Mahaffy, J.S. Halekas, R.C. Elphic, G.T. Delory, Variability of helium, neon, and argon in the lunar exosphere as observed by the LADEE NMS instrument. Geophys. Res. Lett. (2015). https://doi.org/10.1002/2015GL064120

K.R. Bermingham, E. Füri, K. Lodders, B. Marty, The NC-CC isotope dichotomy: Implications for the chemical and isotopic evolution of the early Solar System. Space Sci. Rev. (2020). https://doi.org/10.1007/s11214-020-00748-w, in press

Y. Bétrémieux, L. Kaltenegger, Transmission spectrum of Earth as a transiting exoplanet-from the ultraviolet to the near-infrared. Astrophys. J. 772, L31 (2013). https://doi.org/10.1088/2041-8205/772/2/L31

Th. Bida, R. Killen, T.H. Morgan, Discovery of calcium in Mercury's atmosphere. Nature 404, 159 (2000)

E.B. Bierhaus, B.C. Clark, J.W. Harris, K.S. Payne, R.D. Dubisher, D.W. Wurts, R.A. Hund, R.M. Kuhns, T.M. Linn, J.L. Wood, A.J. May, J.P. Dworkin, E. Beshore, D.S. Lauretta (the OSIRIS-REx Team), The OSIRIS-REx spacecraft and the Touch-and-GoSample Acquisition Mechanism (TAGSAM). Space Sci. Rev. (2018). https://doi.org/10.1007/s11214-018-0521-6

A.S. Bonomo, L. Zeng, M. Damasso et al., A giant impact as the likely origin of different twins in the Kepler107 exoplanet system. Nat. Astron. 3, 416-423 (2019)

F. Bouchy et al., Near-InfraRed planet searcher to join HARPS on the near-InfraRed: ESO 3.6-metre telescope ESO 3.6-metre telescope. Messenger 169, 21 (2017)

L.G. Bouma et al., Planet detection simulations for several possible TESS extended missions (2017). arXiv: 1705.08891

J. Bouret, C. Neiner, A.I.G. de Castro, C. Evans, B. Gaensicke, S. Shore, L. Fossati, C. Gry, S. Charlot, F. Marin, P. Noterdaeme, J.-Y. Chaufray, The science case for POLLUX, a high-resolution UV spectropolarimeter onboard LUVOIR. Proceedings of the SPIE (2018). https://doi.org/10.1117/12.2312621

V. Bourrier, X. Dumusque, C. Dorn et al., The 55 Cancri system reassessed. Astron. Astrophys. 619, A1 (2018)

D. Briot, J. Schneider, Occurrence, physical conditions, and observations of super-Ios and hyper-Ios, in Pathways Towards Habitable Planets, vol. 430 (2010), p. 409

R.A. Brown, A model of Jupiter's sulfur nebula. Astrophys. J. 206, L179 (1976)

R.A. Brown, F.H. Chaffee Jr., High-resolution spectra of sodium emission from Io. Astrophys. J. 187, L125 (1974)

R.A. Brown, D.E. Shemansky, On the nature of S II emission from Jupiter's hot plasma torus. Astrophys. J. 263, 433 (1982)

R.A. Brown, R.M. Goody, F.J. Murcray, F.H. Chaffee Jr., Further studies of line emission from Io. Astrophys. J. 200, L49 (1975)

J.L. Burch, T.E. Moore, R.B. Torbert, B.L. Giles, Magnetospheric multiscale overview and science objectives. Space Sci. Rev. (2016). https://doi.org/10.1007/s11214-015-0164-9

M.H. Burger, R.M. Killen, R.J. Vervack, E.T. Bradley, W.E. McClintock, M. Sarantos, M. Benna, N. Mouawad, Monte Carlo modeling of sodium in Mercury's exosphere during the first two MESSENGER flybys. Icarus (2010). https://doi.org/10.1016/j.icarus.2010.05.007

T.M. Burinskaya, Non-monotonic potentials above the day-side lunar surface exposed to the solar radiation. Planet. Space Sci. (2015). https://doi.org/10.1016/j.pss.2015.03.004

J. Cao, Y. Ma, G. Parks, H. Rème, I. Dandouras, T. Zhang, Kinetic analysis of the energy transport of bursty bulk flows in the plasma sheet. J. Geophys. Res. (2013). https://doi.org/10.1029/2012JA018351

L. Carigi, Solar neighborhood, in Encyclopedia of Astrobiology (Springer, Berlin, 2015), pp. $2286-2287$. https://doi.org/10.1007/978-3-662-44185-5_1460

J. Carpenter, C. Speak, A. Chouker, M. Talevi, R. Nakamura, A. Santangelo, I. Crawford, D. Cullen, B. Bussey, J. Grenouilleau, Research Opportunities on the Deep Space Gateway: Findings from the Workshop and Call for Ideas (2018). ESA-HSO-K-RP-0284

P. Cartigny, B. Marty, Nitrogen isotopes and mantle geodynamics: the emergence of life and the atmospherecrust-mantle connection. Elements 9, 359-366 (2013). https://doi.org/10.2113/gselements.9.5.359 
J. Catanzarite, M. Shao, The occurrence rate of Earth analog planets orbiting Sun-like stars. Astrophys. J. 738 (2011). https://doi.org/10.1088/0004-637X/738/2/151

D.C. Catling, The great oxidation event transition, in Treatise on Geochemistry (Elsevier, Amsterdam, 2014), pp. 177-195. https://doi.org/10.1016/B978-0-08-095975-7.01307-3

D.C. Catling, C.R. Glein, K.J. Zahnle, C.P. McKay, Why $\mathrm{O}_{2}$ is required by complex life on habitable planets and the concept of planetary "oxygenation time". Astrobiology 8, 377-395 (2005). https://doi.org/10.1089/ast.2005.5.415

W.L. Chameides, J.C.G. Walker, Rates of fixation by lightning of carbon and nitrogen in possible primitive atmospheres. Orig. Life Evol. Biosph. 11, 291-302 (1981). https://doi.org/10.1007/BF00931483

Q.H.S. Chan, R. Stroud, Z. Martins, H. Yabuta, Concerns of organic contamination for sample return space missions. Space Sci. Rev. (2020). https://doi.org/10.1007/s11214-020-00678-7

C.R. Chappell, The role of the ionosphere in providing plasma to the terrestrial magnetosphere - an historical overview. Space Sci. Rev. (2015). https://doi.org/10.1007/s11214-015-0168-5

E. Chassefière et al., PHEBUS: a double ultraviolet spectrometer to observe Mercury's exosphere. Planet. Space Sci. (2010). https://doi.org/10.1016/j.pss.2008.05.018

E. Chassefière, R. Wieler, B. Marty, F. Leblanc, The evolution of Venus: present state of knowledge and future exploration. Planet. Space Sci. (2012). https://doi.org/10.1016/j.pss.2011.04.007

S.P. Christon, G. Gloeckler, D.J. Williams, T. Mukai, R.W. McEntire, C. Jacquey, V. Angelopoulos, A.T.Y. Lui, S. Kokubun, D.H. Fairfield, M. Hirahara, T. Yamamoto, Energetic atomic and molecular ions of ionospheric origin observed in distant magnetotail flow-reversal events. Geophys. Res. Lett. 21(25), 3023-3026 (1994)

C. Chyba, C. Sagan, Endogenous production, exogenous delivery and impact-shock synthesis of organic molecules: an inventory for the origins of life. Nature 355, 125-132 (1992). https://doi.org/ 10.1038/355125a0

J.T. Clarke et al., Variability of D and H in the Martian upper atmosphere observed with the MAVEN IUVS echelle channel. J. Geophys. Res. Space Phys. 122, 2336-2344 (2017). https://doi.org/10.1002/ 2016JA023479

C.S. Cockell, L. Kaltenegger, J.A. Raven, Cryptic photosynthesis-extrasolar planetary oxygen without a surface biological signature. Astrobiology 9, 623-636 (2009). https://doi.org/10.1089/ast.2008.0273

V. Cooray, 19. Interaction of lightning flashes with the Earth's atmosphere, in An Introduction to Lightning (Springer, Dordrecht, 2015), pp. 1-386. https://doi.org/10.1007/978-94-017-8938-7

R. Cosentino et al. Harps-N: the new planet hunter at TNG. Proc. SPIE 8446, 84461V (2012)

A. Crida, R. Ligi, C. Dorn, Y. Lebreton, Mass, radius, and composition of the transiting planet 55 Cancri e: using interferometry and correlations. Astrophys. J. 860, 122 (2018)

F. Dai, J.N. Winn, D. Gandolfi et al., The discovery and mass measurement of a new USP: K2-131b. Astron. J. 154, 226 (2017)

J. Dalcanton, S. Seager, S. Aigrain, S. Battel, S. Brant, C. Conroy, L. Feinberg, S. Gezari, O. Guyon, W. Harris, C. Hirata, J. Mather, M. Postman, D. Redding, D. Schiminovich, H.P. Stahl, J. Tumlison, From Cosmic Birth to Living Earths: The Future of UVOIR Space Astronomy. AURA Report (2015). arXiv: 1507.04779

I. Dandouras, Detection of a plasmaspheric wind in the Earth's magnetosphere by the Cluster spacecraft. Ann. Geophys. 31, 1143-1153 (2013). https://doi.org/10.5194/angeo-31-1143-2013

I. Dandouras, M. Yamauchi, J. De Keyser, O. Marghitu, H. Rème, I. Yoshikawa, T. Sakanoi, (the ESCAPE proposal team), ESCAPE: a mission proposal for ESA-M5 to systematically study exosphere and atmospheric escape using European, Japanese, and US instruments, in Proc. ISAS Symposium, Japan (2018). https://repository.exst.jaxa.jp/dspace/handle/a-is/876320

I. Dandouras, R.A. Bamford, G. Branduardi-Raymont, D. Constantinescu, J. De Keyser, Y. Futaana, H. Lammer, F. Leblanc, A. Milillo, R. Nakamura, E. Roussos, M.G.G.T. Taylor, J. Carpenter, Space plasma physics science opportunities for the deep space gateway, in EGU General Assembly, vol. 2019 (2019), EGU2019-9690

I. Dandouras, A.R. Poppe, M.O. Fillingim, L.M. Kistler, C.G. Mouikis, H. Rème, P. Garnier, P. Pinet, G.K. Parks, First simultaneous detection of terrestrial ionospheric heavy ions in the Earth's inner magnetosphere and at the Moon. Nat. Commun. (2020), submitted. NCOMMS-18-24769-T

A. Debrecht, J. Carroll-Nellenback, A. Frank et al., Generation of a circumstellar gas disc by hot Jupiter WASP-12b. Astrophys. J. 478, 2592 (2018)

D.C. Delcourt, J.A. Sauvaud, T.E. Moore, Polar wind ion dynamics in the magnetotail. J. Geophys. Res. 98, 9155-9169 (1993). https://doi.org/10.1029/93JA00301

D. Delcourt, Y. Saito, J.-M. Illiano, N. Krupp et al., The mass spectrum analyzer (MSA) onboard BEPI COLOMBO MMO: scientific objectives and prototype results. Adv. Space Res. (2009). https://doi.org/10.1016/j.asr.2008.12.002 
D. Deming, H. Knutson, J. Kammer et al., Spitzer secondary eclipses of the dense, modestly-irradiated, giant exoplanet HAT-P-20b using pixel-level decorrelation. Astrophys. J. 805, 132 (2015)

B.-O. Demory, M. Gillon, J. de Wit et al., A map of the large day-night temperature gradient of a super-Earth exoplanet. Nature 532, 207 (2016a)

B.-O. Demory, M. Gillon, N. Madhusudhan, D. Queloz, Variability in the super-Earth 55 Cancri e. Mon. Not. R. Astron. Soc. 455, 2018 (2016b)

B.W. Denevi et al., The evolution of Mercury's crust: a global perspective from MESSENGER. Science 324, 613-618 (2009)

D.J. Des Marais, M.O. Harwit, K.W. Jucks, J.F. Kasting, D.N.C. Lin, J.I. Lunine, J. Schneider, S. Seager, W.A. Traub, N.J. Woolf, Remote sensing of planetary properties and biosignatures on extrasolar terrestrial planets. Astrobiology 2, 153-181 (2002). https://doi.org/10.1089/15311070260192246

S.D. Domagal-Goldman, V.S. Meadows, M.W. Claire, J.F. Kasting, Using biogenic sulfur gases as remotely detectable biosignatures on anoxic planets. Astrobiology 11, 419-441 (2011). https://doi.org/ 10.1089/ast.2010.0509

C. Dorn, J.H.D. Harrison, A. Bonsor, T.O. Hands, A new class of super-Earths formed from high-temperature condensates: HD219134 b, 55 Cancri e, WASP-47 e. Mon. Not. R. Astron. Soc. 484, 712 (2019)

X. Dumusque, A.S. Bonomo, R.D. Haywood et al., The Kepler-10 planetary system revisited by HARPS-N: a hot rocky world and a solid Neptune-mass planet. Astrophys. J. 789, 154 (2014)

S. Dyadechkin, E. Kallio, P. Wurz, New fully kinetic model for the study of electric potential, plasma and dust above lunar landscapes. J. Geophys. Res. 120, 1589-1606 (2015). https://doi.org/ 10.1002/2014JA020511

Y. Ebihara, M. Yamada, S. Watanabe, M. Ejiri, Fate of outflowing suprathermal oxygen ions that originate in the polar ionosphere. J. Geophys. Res. 111, A04219 (2006). https://doi.org/10.1029/ 2005JA011403

C.S. Edwards, B.L. Ehlmann, Carbon sequestration on Mars. Geology 43 (2015). https://doi.org/10.1130/ G36983.1

L.T. Elkins-Tanton, Coreless terrestrial exoplanets. Astrophys. J. 688, 628 (2008)

L.T. Elkins-Tanton, S. Seager, Ranges of atmospheric mass and composition of super-Earth exoplanets. Astrophys. J. 685, 1237 (2008)

R.C. Elphic, G.T. Delory, B.P. Hine, P.R. Mahaffy, M. Horanyi, A. Colaprete, M. Benna, S.K. Noble, The lunar atmosphere and dust environment explorer mission. Space Sci. Rev. (2014). https://doi.org/ 10.1007/s11214-014-0113-z

E. Engwall, Low-Energy Ion Escape from the Terrestrial Polar Regions. Ph.D. thesis, Uppsala University, Department of Physics and Astronomy (2009)

E. Engwall, A.I. Eriksson, C.M. Cully, M. André, R. Torbert, H. Vaith, Earth's ionospheric outflow dominated by hidden cold plasma. Nat. Geosci. 2, 24-27 (2009). https://doi.org/10.1038/ngeo387

C.P. Escoubet, M. Fehringer, M. Goldstein, The Cluster mission. Ann. Geophys. 19, 1197-1200 (2001)

C.P. Escoubet, A. Masson, H. Laakso, M.L. Goldstein, Recent highlights from Cluster, the first 3-D magnetospheric mission. Ann. Geophys. (2015). https://doi.org/10.5194/angeo-33-1221-2015

N. Espinoza, R. Brahm, T. Henning et al., HD 213885b: a transiting 1-day-period super-Earth with an Earthlike composition around a bright $(\mathrm{V}=7.9)$ star unveiled by TESS (2019). arXiv:1903.07694

B. Fegley, R.G. Prinn, H. Hartman, G.H. Watkins, Chemical effects of large impacts on the Earth's primitive atmosphere. Nature 319, 305-308 (1986). https://doi.org/10.1038/319305a0

Y.R. Fernández, S.S. Sheppard, D.C. Jewitt, The albedo distribution of Jovian Trojan asteroids. Astron. J. 126, 1563 (2003)

A. Fortier, T. Beck, W. Benz, C. Broeg, V. Cessa, D. Ehrenreich, N. Thomas, Proc. SPIE 91432, (2014)

L. Fossati, T.R. Ayres, C.A. Haswell et al., Absorbing gas around the WASP-12 planetary system. Astrophys. J. Lett. 766, L20 (2013)

L. Fossati, N.V. Erkaev, H. Lammer et al., Aeronomical constraints to the minimum mass and maximum radius of hot low-mass planets. Astron. Astrophys. 598, A90 (2017)

D. Fowler, J.A. Pyle, J.A. Raven, M.A. Sutton, The global nitrogen cycle in the twenty-first century: introduction. Philos. Trans. R. Soc. Lond. B, Biol. Sci. 368, 20130165 (2013). https://doi.org/ 10.1098/rstb.2013.0165

K. France, B. Fleming, G. West, S.R. McCandliss, M.R. Bolcar, W. Harris, L. Moustakas, J.M. O’Meara, I. Pascucci, J. Rigby, D. Schiminovich, J. Tumlinson, J.-C. Bouret, C.J. Evans, M. Garcia, The LUVOIR Ultraviolet Multi-Object Spectrograph (LUMOS): instrument definition and design (2017)

W. Fujiya, P. Hoppe, U. Ott, Boron abundances and isotopic ratios of olivine grains on Itokawa returned by the Hayabusa spacecraft. Meteorit. Planet. Sci. 51(9), 1721-1729 (2016). https://doi.org/ 10.1111/maps. 12686

Y. Futaana et al., SELMA mission: how do airless bodies interact with space environment? The Moon as an accessible laboratory. Planet. Space Sci. (2018). https://doi.org/10.1016/j.pss.2017.11.002 
Gaia Collaboration, VizieR Online Data Catalog. SIMBAD I/345 (2018)

F. Gaillard, B. Scaillet, A theoretical framework for volcanic degassing chemistry in a comparative planetology perspective and implications for planetary atmospheres. Earth Planet. Sci. Lett. 403, 307 (2014)

J.N. Galloway, The global nitrogen cycle, in Treatise on Geochemistry (Elsevier, Amsterdam, 2003), pp. 557-583. https://doi.org/10.1016/B0-08-043751-6/08160-3

D. Gandolfi, O. Barragán, A.P. Hatzes et al., The transiting multi-planet system HD 3167: a 5.7 MEarth super-Earth and an 8.3 MEarth mini-Neptune. Astron. J. 154, 123 (2017)

B.S. Gaudi, S. Seager, B. Mennesson, A. Kiessling, K. Warfield, G. Kuan, K. Cahoy, J.T. Clarke, S. Domagal-Goldman, L. Feinberg, O. Guyon, J. Kasdin, D. Mawet, T. Robinson, L. Rogers, P. Scowen, R. Somerville, K. Stapelfeldt, C. Stark, D. Stern, M. Turnbull, S. Martin, O. Alvarez-Salazar, R. Amini, W. Arnold, B. Balasubramanian, M. Baysinger, L. Blais, T. Brooks, R. Calvet, V. Cormarkovic, C. Cox, R. Danner, J. Davis, L. Dorsett, M. Effinger, R. Eng, J. Garcia, J. Gaskin, J. Harris, S. Howe, B. Knight, J. Krist, D. Levine, M. Li, D. Lisman, M. Mandic, L. Marchen, C. MarreseReading, J. McGowen, A. Miyaguchi, R. Morgan, B. Nemati, S. Nikzad, J. Nissen, M. Novicki, T. Perrine, D. Redding, M. Richards, M. Rud, D. Scharf, G. Serabyn, S. Shaklan, S. Smith, M. Stahl, P. Stahl, H. Tang, D. Van Buren, J. Villalvazo, S. Warwick, D. Webb, R. Wofford, J. Woo, M. Wood, J. Ziemer, E. Douglas, V. Faramaz, S. Hildebrandt, T. Meshkat, P. Plavchan, G. Ruane, N. Turner (2018). The Habitable Exoplanet Observatory (HabEx) Mission Concept Study Interim Report. https://www.jpl.nasa.gov/habex/pdf/HabEx_Interim_Report.pdf

S. Gaudi, S. Seager, B. Mennesson, A. Kiessling, K. Warfield, The Habitable Exoplanet Observatory (HabEx), in SPIE-Intl. Soc. Optical Eng., p. 21 (2019). https://doi.org/10.1117/12.2530036

J. Geiss, F. Bühler, H. Cerutti, P. Eberhardt, Ch. Filleux, J. Meister, P. Signer, The Apollo SWC experiment: results, conclusions, consequences. Space Sci. Rev. (2004). https://doi.org/10.1023/ B:SPAC.0000023409.54469.40

A. Genova et al., Geodetic evidence that Mercury has a solid inner core. Geophys. Res. Lett. (2019). https://doi.org/10.1029/2018GL081135

L. Girardi, A. Bressan, G. Bertelli, C. Chiosi, Evolutionary tracks and isochrones for low- and intermediatemass stars: from 0.15 to $7 \mathrm{M}_{\text {sun }}$, and from $\mathrm{Z}=0.0004$ to 0.03 . Astron. Astrophys. Suppl. Ser. 141, 371-383 (2000). https://doi.org/10.1051/aas:2000126

J.N. Goswami, Exploration of the Moon: Chandrayaan-1 and Chandrayaan-2, in 40th COSPAR Scientific Assembly (2014). B0.1-7-14

M.M. Grady, Exploring Mars with returned samples. Space Sci. Rev. (2020). https://doi.org/10.1007/ s11214-020-00676-9

J.L. Grenfell, J.-M. Grießmeier, B. Patzer, H. Rauer, A. Segura, A. Stadelmann, B. Stracke, R. Titz, P. Von Paris, Biomarker response to galactic cosmic ray-induced NOx and the methane greenhouse effect. Astrobiology 7, 208-221 (2007a). https://doi.org/10.1089/ast.2006.0129. In The Atmosphere of an EarthLike Planet Orbiting an M Dwarf Star

J.L. Grenfell, B. Stracke, P. von Paris, B. Patzer, R. Titz, A. Segura, H. Rauer, The response of atmospheric chemistry on earthlike planets around F, G and K Stars to small variations in orbital distance. Planet. Space Sci. 55, 661-671 (2007b). https://doi.org/10.1016/j.pss.2006.09.002

J.L. Grenfell, H. Rauer, F. Selsis, L. Kaltenegger, C. Beichman, W. Danchi, C. Eiroa, M. Fridlund, T. Henning, T. Herbst, H. Lammer, A. Léger, R. Liseau, J.I. Lunine, F. Paresce, A. Penny, A. Quirrenbach, H. Röttgering, J. Schneider, D. Stam, G. Tinetti, G.J. White, Co-evolution of atmospheres, life, and climate. Astrobiology 10, 77-88 (2010). https://doi.org/10.1089/ast.2009.0375

J.L. Grenfell, J.-M. Grießmeier, P. von Paris, A.B.C. Patzer, H. Lammer, B. Stracke, S. Gebauer, F. Schreier, H. Rauer, Response of Atmospheric Biomarkers to NOx-Induced Photochemistry Generated by Stellar Cosmic Rays for Earth-like Planets in the Habitable Zone of M Dwarf Stars. Astrobiology 12, 11091122 (2012). https://doi.org/10.1089/ast.2011.0682,

M. Güdel, The Sun through time. Space Sci. Rev. (2020), accepted

E.W. Guenther, J. Cabrera, A. Erikson et al., Constraints on the exosphere of CoRoT-7b. Astron. Astrophys. 525, A24 (2011)

E.W. Guenther, O. Barragán, F. Dai et al., K2-106, a system containing a metal-rich planet and a planet of lower density. Astron. Astrophys. 608, A93 (2017)

A. Guzmán-Marmolejo, A. Segura, El. Escobar-Briones, Abiotic production of methane in terrestrial planets. Astrobiology 13(6), 550-559 (2013). https://doi.org/10.1089/ast.2012.0817

S. Haaland, A. Eriksson, E. Engwall, B. Lybekk, H. Nilsson, A. Pedersen, K. Svenes, M. André, M. Förster, K. Li, C. Johnsen, N. Østgaard, Estimating the capture and loss of cold plasma from ionospheric outflow. J. Geophys. Res. 117, A07311 (2012). https://doi.org/10.1029/2012JA017679

T. Haltigin et al., iMARS Phase 2: a draft mission architecture and science management plan for the return of samples from Mars. Astrobiology 18 (2018). https://doi.org/10.1089/ast.2018.29027.mars 
Y. Harada, Y. Futaana, S. Barabash, M. Wieser, P. Wurz, A. Bhardwaj, K. Asamura, Y. Saito, S. Yokota, H. Tsunakawa, S. Machida, Backscattered energetic neutral atoms from the Moon in the Earth's plasma sheet observed by Chandarayaan-1/sub-keV atom reflecting analyzer instrument. J. Geophys. Res. 119, 3573-3584 (2014). https://doi.org/10.1002/2013JA019682

M.H. Hart, Habitable zones about main sequence stars. Icarus 37, 351-357 (1979). https://doi.org/ 10.1016/0019-1035(79)90141-6

C.A. Haswell, L. Fossati, T. Ayres et al., Near-ultraviolet absorption, chromospheric activity, and star-planet interactions in the WASP-12 system. Astrophys. J. 760, 79 (2012)

R.D. Haywood, A. Collier Cameron, D. Queloz et al., Planets and stellar activity: hide and seek in the CoRoT7 system. Mon. Not. R. Astron. Soc. 443, 2517 (2014)

L. Hebb, A. Collier-Cameron, B. Loeillet et al., WASP-12b: the hottest transiting extrasolar planet yet discovered. Astrophys. J. 693, 1920 (2009)

P. Hedelt, P. von Paris, M. Godolt, S. Gebauer, J.L. Grenfell, H. Rauer, F. Schreier, F. Selsis, T. Trautmann, Spectral features of Earth-like planets and their detectability at different orbital distances around F, G, and K-type stars. Astron. Astrophys. 553, A9 (2013). https://doi.org/10.1051/0004-6361/201117723

M. Hippke, D. Angerhausen, A statistical search for a population of exo-trojans in the Kepler data set. Astrophys. J. 881, 1 (2015)

G.C. Ho, S.M. Krimigis, R.E. Gold, D.N. Baker, J.A. Slavin, B.J. Anderson, H. Korth, R.D. Starr, D.J. Lawrence, R.L. McNutt, S.C. Solomon, MESSENGER observations of transient bursts of energetic electrons in Mercury's magnetosphere. Science (2011). https://doi.org/10.1126/science.1211141

R.R. Hodges Jr., Methane in the lunar exosphere: implications for solar wind carbon escape. Geophys. Res. Lett. (2016). https://doi.org/10.1002/2016GL068994

A.W. Howard, R. Sanchis-Ojeda, G.W. Marcy et al., A rocky composition for an Earth-sized exoplanet. Nature 503, 381 (2013)

M.M. Huddleston, C.R. Chappell, D.C. Delcourt, T.E. Moore, B.L. Giles, M.O. Chandler, An examination of the process and magnitude of ionospheric plasma supply to the magnetosphere. J. Geophys. Res. (2005). https://doi.org/10.1029/2004JA010401

R. Ilie, M.W. Liemohn, The outflow of ionospheric nitrogen ions: a possible tracer for the altitudedependent transport and energization processes of ionospheric plasma. J. Geophys. Res. (2016). https://doi.org/10.1002/2015JA022162

T.R. Ireland, P. Holden, M.D. Norman, J. Clarke, Isotopic enhancements of ${ }^{17} \mathrm{O}$ and ${ }^{18} \mathrm{O}$ from solar wind particles in the lunar regolith. Nature Lett. (2006). https://doi.org/10.1038/nature04611

Y. Ito, M. Ikoma, H. Kawahara et al., Theoretical emission spectra of atmospheres of hot rocky super-Earths. Astrophys. J. 801, 144 (2015)

B.M. Jakosky, R.P. Lin, J.M. Grebowsky, J.G. Luhmann et al., The Mars Atmosphere and Volatile Evolution (MAVEN) mission. Space Sci. Rev. 195, 3-48 (2015a). https://doi.org/10.1007/s11214-015-0139-X

B.M. Jakosky, J.M. Grebowsky, J.G. Luhmann, J. Connerney et al., MAVEN observations of the response of Mars to an interplanetary coronal mass ejection. Science 350, aad0210 (2015b). https:// doi.org/10.1126/science.aad0210

B.M. Jakosky, J.M. Grebowsky, J.G. Luhmann, D.A. Brain, Initial results from the MAVEN mission to Mars. Geophys. Res. Lett. 42, 8791-8802 (2015c). https://doi.org/10.1002/2015GL065271

B.M. Jakosky, M. Slipski, M. Benna, P. Mahaffy, M. Elrod, R. Yelle, S. Stone, N. Alsaeed, Mars' atmospheric history derived from upper-atmosphere measurements of 38Ar/36Ar. Science 355, 1408-1410 (2017)

B.M. Jakosky, D. Brain, M. Chaffin, S. Curry, J. Deighan, J. Grebowsky, J. Halekas, F. Leblanc, R. Lillis, J.G. Luhmann, L. Andersson, N. Andre, D. Andrews, D. Baird, D. Baker, J. Bell, M. Benna, D. Bhattacharyya, S. Bougher, C. Bowers, P. Chamberlin, J.-Y. Chaufray, J. Clarke, G. Collinson, M. Combi, J. Connerney, K. Connour, J. Correira, K. Crabb, F. Crary, T. Cravens, M. Crismani, G. Delory, R. Dewey, G. DiBraccio, C. Dong, Y. Dong, P. Dunn, H. Egan, M. Elrod, S. England, F. Eparvier, R. Ergun, A. Eriksson, T. Esman, J. Espley, S. Evans, K. Fallows, X. Fang, M. Fillingim, C. Flynn, A. Fogle, C. Fowler, J. Fox, M. Fujimoto, P. Garnier, Z. Girazian, H. Groeller, J. Gruesbeck, O. Hamil, K.G. Hanley, T. Hara, Y. Harada, J. Hermann, M. Holmberg, G. Holsclaw, S. Houston, S. Inui, S. Jain, R. Jolitz, A. Kotova, T. Kuroda, D. Larson, Y. Lee, C. Lee, F. Lefevre, C. Lentz, D. Lo, R. Lugo, Y.-J. Ma, P. Mahaffy, M.L. Marquette, Y. Matsumoto, M. Mayyasi, C. Mazelle, W. McClintock, J. McFadden, A. Medvedev, M. Mendillo, K. Meziane, Z. Milby, D. Mitchell, R. Modolo, F. Montmessin, A. Nagy, H. Nakagawa, C. Narvaez, K. Olsen, D. Pawlowski, W. Peterson, A. Rahmati, K. Roeten, N. Romanelli, S. Ruhunusiri, C. Russell, S. Sakai, N. Schneider, K. Seki, R. Sharrar, S. Shaver, D.E. Siskind, M. Slipski, Y. Soobiah, M. Steckiewicz, M.H. Stevens, I. Stewart, A. Stiepen, S. Stone, V. Tenishev, N. Terada, K. Terada, E. Thiemann, R. Tolson, G. Toth, J. Trovato, M. Vogt, T. Weber, P. Withers, S. Xu, R. Yelle, E. Yiğit, R. Zurek, Loss of the Martian atmosphere to space: present-day loss rates determined from MAVEN observations and integrated loss through time. Icarus (2018). https://doi.org/10.1016/j.icarus.2018.05.030

C.Y. Johnson, Ion and neutral composition of the ionosphere. Ann. IQSY 5, 197-213 (1969) 
B. Johnson, C. Goldblatt, The nitrogen budget of Earth. Earth-Sci. Rev. 148, 150-173 (2015). https://doi.org/10.1016/j.earscirev.2015.05.006

E. Kallio, G. Facskó, Properties of plasma near the moon in the magnetotail. Planet. Space Sci. (2015). https://doi.org/10.1016/j.pss.2014.11.007

E. Kallio, S. Dyadechkin, P. Wurz, M. Khodachenko, Space weathering on the Moon: farside-nearside solar wind precipitation asymmetry. Planet. Space Sci. 166, 9-22 (2019). https://doi.org/10.1016/ j.pss.2018.07.013

L. Kaltenegger, W.A. Traub, Transits of Earth-like planets. Astrophys. J. (2009). https://doi.org/10.1088/ 0004-637X/698/1/519

J.F. Kasting, How to Find a Habitable Planet (Princeton University Press, Princeton, 2010). ISBN 978-0691-13805-3

J.F. Kasting, D.P. Whitmire, R.T. Reynolds, Habitable zones around main sequence stars. Icarus 101, 108-128 (1993). https://doi.org/10.1006/icar.1993.1010

J.W. Keller, N.E. Petro, R.R. Vondrak (the LRO team), The lunar reconnaissance orbiter mission - six years of science and exploration at the Moon. Icarus (2016). https://doi.org/10.1016/j.icarus.2015.11.024

R.M. Killen, J.M. Hahn, Impact vaporization as a possible source of Mercury's calcium exosphere. Icarus (2015). https://doi.org/10.1016/j.icarus.2014.11.035

R. Killen, W.-H. Ip, The surface-bounded atmospheres of Mercury and the Moon. Rev. Geophys. 37(3), 361406 (1999). https://doi.org/10.1029/1999RG900001

R. Killen, G. Cremonese, H. Lammer, S. Orsini, A.E. Potter, A.L. Sprague, P. Wurz, M.L. Khodachenko, H.I.M. Lichtenegger, A. Milillo, A. Mura, Processes that promote and deplete the exosphere of Mercury. Space Sci. Rev. 132, 433-509 (2007)

R.M. Killen, A.E. Potter, R.J. Vervack Jr., E.T. Bradley, W.E. McClintock, C.M. Anderson, M.H. Burger, Observations of metallic species in Mercury's exosphere. Icarus (2010). https://doi.org/10.1016/ j.icarus.2010.02.018

K.G. Kislyakova, E. Pilat-Lohinger, B. Funk et al., On the ultraviolet anomalies of the WASP-12 and HD 189733 systems: Trojan satellites as a plasma source. Mon. Not. R. Astron. Soc. 461, 988 (2016)

K.G. Kislyakova, L. Noack, C.P. Johnstone et al., Magma oceans and enhanced volcanism on TRAPPIST-1 planets due to induction heating. Nat. Astron. 1, 878 (2017)

K.G. Kislyakova, L. Fossati, C.P. Johnstone et al., Effective induction heating around strongly magnetized stars. Astrophys. J. 858, 105 (2018)

L.M. Kistler, C. Mouikis, E. Möbius, B. Klecker, J.A. Sauvaud, H. Rème, A. Korth, M.F. Marcucci, R. Lundin, G.K. Parks, A. Balogh, Contribution of nonadiabatic ions to the cross-tail current in an $\mathrm{O}^{+}$ dominated thin current sheet. J. Geophys. Res. (2005). https://doi.org/10.1029/2004JA010653

E.S. Kite, B. Fegley Jr., L. Schaefer, E. Gaidos, Atmosphere-interior exchange on hot, rocky exoplanets. Astrophys. J. 828, 80 (2016)

R.K. Kopparapu, R. Ramirez, J.F. Kasting, V. Eymet, T.D. Robinson, S. Mahadevan, R.C. Terrien, S. Domagal-Goldman, V. Meadows, R. Deshpande, Habitable zones around main sequence stars: new estimates. Astrophys. J. 765, 131 (2013). https://doi.org/10.1088/0004-637X/765/2/131

V.A. Krasnopolsky, J.P. Maillard, T.C. Owen, Detection of methane in the Martian atmosphere: evidence for life? Icarus 172, 537-547 (2004). https://doi.org/10.1016/j.icarus.2004.07.004

J. Krissansen-Totton, E.W. Schwieterman, B.D. Charnay, G.N. Arney, T.D. Robinson, V. Meadows, D.C. Catling, Is the pale blue dot unique? Optimized photometric bands for identifying Earth-like exoplanets. Astrophys. J. 817, 31 (2016). https://doi.org/10.3847/0004-637X/817/1/31

J. Krissansen-Totton, G.N. Arney, D.C. Catling, Constraining the climate and ocean pH of the early Earth with a geological carbon cycle model. Proc. Natl. Acad. Sci. 115(16), 4105-4110 (2018). https:// doi.org/10.1073/pnas.1721296115

E.A. Kronberg, M. Ashour-Abdalla, I. Dandouras, D.C. Delcourt, E.E. Grigorenko, L.M. Kistler, I.V. Kuzichev, J. Liao, R. Maggiolo, H.V. Malova, K.G. Orlova, V. Peroomian, D.R. Shklyar, Y.Y. Shprits, D.T. Welling, L.M. Zelenyi, Circulation of heavy ions and their dynamical effects in the magnetosphere: recent observations and models. Space Sci. Rev. (2014). https://doi.org/10.1007/s11214-014-0104-0

M. Kruss, G. Wurm, Seeding the formation of Mercurys: an iron-sensitive bouncing barrier in disk magnetic fields. Astrophys. J. 869, 45 (2018)

D. Kubyshkina, L. Fossati, N.V. Erkaev et al., Grid of upper atmosphere models for 1-40 MEarth planets: application to CoRoT-7 b and HD 219134 b,c. Astron. Astrophys. 619, A151 (2018)

I. Kupo, Y. Mekler, Y. Mekler, A. Eviatar, Detection of ionized sulfur in the Jovian magnetosphere. Astrophys. J. 205, L51 (1976)

M. Küppers, K. Jockers, Fabry-Perot imaging of O III lambda $=5007$ Angstrom Emission in the Io plasma torus. Astrophys. J. 441, L101 (1995)

M. Küppers, K. Jockers, A multi-emission imaging study of the Io plasma torus. Icarus 129, 48 (1997) 
H. Lammer, J.F. Kasting, E. Chassefière, R.E. Johnson, Y.N. Kulikov, F. Tian, Atmospheric escape and evolution of terrestrial planets and satellites. Space Sci. Rev. (2008). https://doi.org/ 10.1007/s11214-008-9413-5

H. Lammer, J.H. Bredehöft, A. Coustenis, M.L. Khodachenko, L. Kaltenegger, O. Grasset, D. Prieur, F. Raulin, P. Ehrenfreund, M. Yamauchi, J.-E. Wahlund, J.-M. Grießmeier, G. Stangl, C.S. Cockell, Y.N. Kulikov, J.L. Grenfell, H. Rauer, What makes a planet habitable? Astron. Astrophys. Rev. 17, 181-249 (2009). https://doi.org/10.1007/s00159-009-0019-z

H. Lammer, H.I.M. Lichtenegger, M.L. Khodachenko, The loss of nitrogen-rich atmospheres from Earth-like exoplanets within M-star habitable zones, in Molecules in the Atmospheres of Extrasolar Planets. ASP Conference Series, vol. 450 (2011), p. 139. Astronomical Society of the Pacific

H. Lammer, A.L. Zerkle, S. Gebauer et al., Origin and evolution of the atmospheres of early Venus, Earth and Mars. Astron. Astrophys. Rev. 26, 2 (2018)

H. Lammer, L. Sproß, J.L. Grenfell, M. Scherf, L. Fossati, M. Lendl, P.E. Cubillos, The role of $\mathrm{N}_{2}$ as a geo-biosignature for the detection and characterization of Earth-like habitats. Astrobiology 19, 927-950 (2019). https://doi.org/10.1089/ast.2018.1914

H. Lammer, M. Scherf, H. Kurokawa, Y. Ueno, C. Burger, T. Maindl, C.P. Johnstone, M. Leizinger, M. Benedikt, L. Fossati, K.G. Kislyakova, B. Marty, G. Avice, B. Fegley, P. Odert, Loss and fractionation of noble gas isotopes and moderately volatile elements from planetary embryos and early Venus, Earth and Mars. Space Sci. Rev. 216, 74 (2020a). https://doi.org/10.1007/s11214-020-00701-X

H. Lammer, R. Brasser, A. Johansen, M. Scherf, M. Leitzinger, Formation of Venus, Earth and Mars: constrained by isotopes. Space Sci. Rev. (2020b), in press

H. Lammer, M. Leitzinger, M. Scherf, P. Odert, C. Burger, D. Kubyshkina, C. Johnstone, T. Maindl, C.M. Schäfer, M. Güdel, N. Tosi, A. Nikolaou, E. Marcq, N.V. Erkaev, L. Noack, K.G. Kislyakova, L. Fossati, E. Pilat-Lohinger, F. Ragossnig, E.A. Dorfi, Constraining the early evolution of Venus and Earth through atmospheric Ar, Ne isotope and bulk K/U ratios. Icarus 339, 113551 (2020c). https://doi.org/10.1016/j.icarus.2019.113551

D.S. Lauretta et al., The OSIRIS-REx target asteroid (101955) Bennu: constraints on its physical, geological, and dynamical nature from astronomical observations. Meteorit. Planet. Sci. 50, 834-849 (2015)

D.S. Lauretta et al., The unexpected surface of asteroid (101955) Bennu. Nature (2019). https://doi.org/ 10.1038/s41586-019-1033-6

F. Leblanc, J.Y. Chaufray, Mercury and Moon He exospheres: analysis and modelling. Icarus (2011). https://doi.org/10.1016/j.icarus.2011.09.028

F. Leblanc, R.E. Johnson, Mercury exosphere. I. Global circulation model of its sodium component. Icarus (2010). https://doi.org/10.1016/j.icarus.2010.04.020

A. Léger, M. Pirre, F.J. Marceau, Search for primitive life on a distant planet: relevance of $\mathrm{O}_{2}$ and $\mathrm{O}_{3}$ detections. Astron. Astrophys. 277, 309 (1993)

A. Léger, D. Rouan, J. Schneider, P. Barge, M. Fridlund, B. Samuel, M. Ollivier, E. Guenther, M. Deleuil, H.J. Deeg et al., Transiting exoplanets from the CoRoT space mission. VIII. CoRoT-7b: the first super-Earth with measured radius. Astron. Astrophys. 506, 287 (2009)

A. Léger, M. Fontecave, A. Labeyrie, B. Samuel, O. Demangeon, D. Valencia, Is the presence of oxygen on an exoplanet a reliable biosignature? Astrobiology 11, 335-341 (2011). https://doi.org/ 10.1089/ast.2010.0516

Z.M. Leinhardt, S.T. Stewart, Collisions between gravity-dominated bodies. I. Outcome regimes and scaling laws. Astrophys. J. 745, 79 (2012). https://doi.org/10.1088/0004-637X/745/1/79

J.S. Levine, The Photochemistry of Atmospheres: Earth, the Other Planets, and Comets (Academic Press, San Diego, 2014)

K. Li, Y. Wei, M. André, A. Eriksson, S. Haaland, E.A. Kronberg, H. Nilsson, L. Maes, Z.J. Rong, W.X. Wan, Cold ion outflow modulated by the solar wind energy input and tilt of the geomagnetic dipole. J. Geophys. Res. 122, 10,658-10,668 (2017). https://doi.org/10.1002/2017JA024642

C. Li, D. Liu, B. Liu, X. Ren, J. Liu, Z. He, W. Zuo, X. Zeng, R. Xu, X. Tan, X. Zhang, W. Chen, R. Shu, W. Wen, Y. Su, H. Zhang, Z. Ouyang, Chang'E-4 initial spectroscopic identification of lunar far-side mantle-derived materials. Nature (2019). https://doi.org/10.1038/s41586-019-1189-0

S. Li, P.G. Lucey, A.A. Fraeman, A.R. Poppe, V.Z. Sun, D.M. Hurley, P.H. Schultz, Widespread hematite at high latitudes of the Moon. Sci. Adv. 6, eaba1940 (2020)

R.J. Lillis et al., The Escape, Plasma and Acceleration Dynamics Explorers (ESCAPADE), a Response to NASA Science Mission Directorate (SMD) Small Innovative Missions for Planetary Exploration (2018). (SIMPLEX-II) program

Y. Liu, L.M. Kistler, C.G. Mouikis, B. Klecker, I. Dandouras, Heavy ion effects on substorm loading and unloading in the Earth's magnetotail. J. Geophys. Res. (2013). https://doi.org/10.1002/jgra.50240

M.K. Lloyd, H.L.O. McClelland, G. Antler, A.S. Bradley, I. Halevy, C.K. Junium, S.D. Wankel, A.L. Zerkle, The isotopic imprint of life on an evolving planet. Space Sci. Rev. 216, 112 (2020). https://doi.org/ 10.1007/s11214-020-00730-6 
J.E. Lovelock, Thermodynamics and the recognition of alien biospheres. Proc. R. Soc. Lond. B, Biol. Sci. (1975). https://doi.org/10.1098/rspb.1975.0051

P. Ludovic, The phase a study of the ESA M4 mission candidate ARIEL. Exp. Astron. 46(1), 211-239 (2018)

N. Madhusudhan, K.K.M. Lee, O. Mousis, A possible carbon-rich interior in super-Earth 55 Cancri e. Astrophys. J. 759, L40 (2012)

S. Massetti, S. Orsini, A. Milillo, A. Mura, E. De Angelis, H. Lammer, P. Wurz, Mapping of the cusp plasma precipitation on the surface of Mercury. Icarus (2003). https://doi.org/10.1016/j.icarus.2003.08.005

S. Massetti, S. Orsini, A. Milillo, A. Mura, Modelling Mercury's magnetosphere and plasma entry through the dayside magnetopause. Planet. Space Sci. (2007). https://doi.org/10.1016/j.pss.2006.12.008

M. Mayor et al., Setting new standards with HARPS. Messenger 114, 20 (2003)

W.E. McClintock, R.J. Vervack Jr., E.T. Bradley, R.M. Killen, N. Mouawad, A.L. Sprague, M.H. Burger, S.C. Solomon, N.R. Izenberg, MESSENGER observations of Mercury's exosphere: detection of magnesium and distribution of constituents. Science (2009). https://doi.org/10.1126/science.1172525

V.S. Meadows, C.T. Reinhard, G.N. Arney, M.N. Parenteau, E.W. Schwieterman, S.D. Domagal-Goldman, A.P. Lincowski, K.R. Stapelfeldt, H. Rauer, S. DasSarma, S. Hegde, N. Narita, R. Deitrick, J. LustigYaeger, T.W. Lyons, N. Siegler, J.L. Grenfell, Exoplanet biosignatures: understanding oxygen as a biosignature in the context of its environment. Astrobiology 18, 630-662 (2018). https://doi.org/ $10.1089 /$ ast.2017.1727

Y. Miguel, Observability of molecular species in a nitrogen dominated atmosphere for 55 Cancri e. Mon. Not. R. Astron. Soc. 482, 2893 (2019)

S. Mikhail, D.A. Sverjensky, Nitrogen speciation in upper mantle fluids and the origin of Earth's nitrogen-rich atmosphere. Nat. Geosci. 7, 816-819 (2014). https://doi.org/10.1038/ngeo2271

A. Milillo, P. Wurz, S. Orsini, D. Delcourt, E. Kallio, R.M. Killen, H. Lammer, S. Massetti, A. Mura, S. Barabash, G. Cremonese, I.A. Daglis, E. Angelis, A.M. Lellis, S. Livi, V. Mangano, K. Torkar, Surface-exosphere-magnetosphere system of Mercury. Space Sci. Rev. (2005). https://doi.org/10.1007/ s11214-005-3593-z

A. Milillo, M. Fujimoto, G. Murakami, J. Benkhoff et al., Investigating Mercury's environment with the twospacecraft BepiColombo mission. Space Sci. Rev. (2020). https://doi.org/10.1007/s11214-020-00712-8

A. Misra, V. Meadows, M.W. Claire, D. Crisp, Using dimers to measure biosignatures and atmospheric pressure for terrestrial exoplanets. Astrobiology 14, 67-86 (2014). https://doi.org/10.1089/ast.2013.0990

J.S. Morgan, C.B. Pilcher, Plasma characteristics of the Io torus. Astrophys. J. 253, 406 (1982)

T. Morota et al., Sample collection from asteroid (162173) Ryugu by Hayabusa2: implications for surface evolution. Science 368, 654-659 (2020). https://doi.org/10.1126/science.aaz6306

C. Muller, $\mathrm{N}_{2} \mathrm{O}$ as a biomarker, from the Earth and solar system to exoplanets, in The Early Evolution of the Atmospheres of Terrestrial Planets, Astrophysics and Space Science Proceedings (Springer, New York, 2013), pp. 99-106. https://doi.org/10.1007/978-1-4614-5191-4_8

A. Mura, P. Wurz, J. Schneider et al., Comet-like tail-formation of exospheres of hot rocky exoplanets: possible implications for CoRoT-7b. Icarus 211, 1 (2011)

T. Nagai, N. Kitamura, H. Hasegawa, I. Shinohara et al., Thick escaping magnetospheric ion layer in magnetopause reconnection with MMS observations. Geophys. Res. Lett. (2016). https://doi.org/ 10.1002/2016GL069085

R. Nakamura, Substorms and their solar wind causes. Space Sci. Rev. (2006). https://doi.org/10.1007/ s11214-006-9131-9

D. Nakashima, N.T. Kita, T. Ushikubo, T. Noguchi, T. Nakamura, J.W. Valley, Oxygen three-isotope ratios of silicate particles returned from asteroid Itokawa by the Hayabusa spacecraft: a strong link with equilibrated LL chondrites. Earth Planet. Sci. Lett. 379, 127-136 (2013). https://doi.org/ 10.1016/j.eps1.2013.08.009

R. Navarro-González, M.J. Molina, L.T. Molina, Nitrogen fixation by volcanic lightning in the early Earth. Geophys. Res. Lett. 25, 3123-3126 (1998). https://doi.org/10.1029/98GL02423

R. Navarro-González, C.P. McKay, D.N. Mvondo, A possible nitrogen crisis for Archean life due to reduced nitrogen fixation by lighting. Nature 412(412), 61-64 (2001)

A. Néri, J. Guignard, M. Monnereau, M.J. Toplis, G. Quitté, Metal segregation in planetesimals: constraints from experimentally determined interfacial energies. Earth Planet. Sci. Lett. 518, 40-52 (2019). https://doi.org/10.1016/j.eps1.2019.04.049

H. Nilsson, M. Waara, S. Arvelius, O. Marghitu, M. Bouhram, Y. Hobara, M. Yamauchi, R. Lundin, H. Rème, J.-A. Sauvaud, I. Dandouras, A. Balogh, L.M. Kistler, B. Klecker, C.W. Carlson, M.B. BavassanoCattaneo, A. Korth, Characteristics of high altitude oxygen ion energization and outflow as observed by Cluster; a statistical study. Ann. Geophys. 24, 1099-1112 (2006)

H. Nilsson, M. Waara, O. Marghitu, M. Yamauchi, R. Lundin, H. Rème, J.-A. Sauvaud, I. Dandouras, E. Lucek, L.M. Kistler, B. Klecker, C.W. Carlson, M.B. Bavassano-Cattaneo, A. Korth, An assessment of the role of the centrifugal acceleration mechanism in high altitude polar cap oxygen ion outflow. Ann. Geophys. 26, 145-157 (2008) 
L.R. Nittler et al., The major-element composition of Mercury's surface from MESSENGER X-ray spectrometry. Science 333, 1847-1850 (2011)

D.P. O'Brien, R. Greenberg, The collisional and dynamical evolution of the main-belt and NEA size distributions. Icarus 178, 179-212 (2005). https://doi.org/10.1016/j.icarus.2005.04.001

C. O'Neill, H.St.C. O'Neill, A.M. Jellinek, On the distribution and variation of radioactive heat producing elements within meteorites, the Earth, and planets. Space Sci. Rev. 216, 37 (2020)

S. Orsini, S. Livi, K. Torkar, S. Barabash, A. Milillo, P. Wurz, A.M. Di Lellis, E. Kallio (the SERENA team), SERENA: a suite of four instruments (ELENA, STROFIO, PICAM and MIPA) on board BepiColombo-MPO for particle detection in the Hermean environment. Planet. Space Sci. (2010). https://doi.org/10.1016/j.pss.2008.09.012

S. Orsini, V. Mangano, A. Milillo, C. Plainaki, A. Mura, J.M. Raines, E. De Angelis, R. Rispoli, F. Lazzarotto, A. Aronica, Mercury sodium exospheric emission as a proxy for solar perturbations transit. Nat. Sci. Rep. (2018). https://doi.org/10.1038/s41598-018-19163-x

T.C. Owen, The search for early forms of life in other planetary systems: future possibilities afforded by spectroscopic techniques, in Strategies for the Search for Life in the Universe, ed. by M.D. Papagiannis (Springer, Dordrecht, 1980), pp. 177-185. https://doi.org/10.1007/978-94-009-9115-6_17

M. Ozima, K. Seki, N. Terada, Y.N. Miura, F.A. Podosek, H. Shinagawa, Terrestrial nitrogen and noble gases in lunar soils. Nature (2005). https://doi.org/10.1038/nature03929

E. Pallé, M.R.Z. Osorio, R. Barrena, P. Montañés-Rodríguez, E.L. Martín, Earth's transmission spectrum from lunar eclipse observations. Nature 459, 814-816 (2009). https://doi.org/10.1038/nature08050

D. Parkos, A. Pikus, A. Alexeenko, H.J. Melosh, HCN production via impact ejecta reentry during the late heavy bombardment. J. Geophys. Res., Planets 123, $892-909$ (2018). https://doi.org/ 10.1002/2017JE005393

G.K. Parks, E. Lee, S. Fu, M. Fillingim, I. Dandouras, Y. Cui, J. Hong, H. Rème, Outflow of low-energy O ${ }^{+}$ ion beams observed during periods without substorms. Ann. Geophys. 33, 333 (2015)

F.A. Pepe et al., ESPRESSO: the Echelle spectrograph for rocky exoplanets and stable spectroscopic observations. Proc. SPIE 7735, 77354Y (2010)

E.A. Petigura, A.W. Howard, G.W. Marcy, Prevalence of Earth-size planets orbiting Sun-like stars. Proc. Natl. Acad. Sci. USA 110, 19273-19278 (2013). https://doi.org/10.1073/pnas.1319909110

S.T. Petsch, The global oxygen cycle, in Treatise on Geochemistry (2014), pp. 437-473. https:// doi.org/10.1016/B978-0-08-095975-7.00811-1

M. Pfleger, H.I.M. Lichtenegger, P. Wurz et al., 3D-modeling of Mercury's solar wind sputtered surfaceexosphere environment. Planet. Space Sci. 115, 90 (2015)

C.B. Pilcher, Biosignatures of early Earths. Astrobiology 3, 471-486 (2003). https://doi.org/10.1089/ 153110703322610582

A.R. Poppe, R. Samad, J.S. Halekas, M. Sarantos, G.T. Delory, W.M. Farrell, V. Angelopoulos, J.P. McFadden, ARTEMIS observations of lunar pick-up ions in the terrestrial magnetotail lobes. Geophys. Res. Lett. (2012). https://doi.org/10.1029/2012GL052909

A.R. Poppe, S. Fatemi, I. Garrick-Bethell, D. Hemingway, M. Holmström, Solar wind interaction with the Reiner Gamma crustal magnetic anomaly: connecting source magnetization to surface weathering. Icarus (2015). https://doi.org/10.1016/j.icarus.2015.11.005

A.R. Poppe, M.O. Fillingim, J.S. Halekas, J. Raeder, V. Angelopoulos, ARTEMIS observations of terrestrial ionospheric molecular ion outflow at the Moon. Geophys. Res. Lett. (2016). https://doi.org/ 10.1002/2016GL069715

A.E. Potter, R.M. Killen, T.H. Morgan, Variation of lunar sodium during passage of the Moon through the Earth's magnetotail. J. Geophys. Res. (2000). https://doi.org/10.1029/1999JE001213

J. Prieto-Arranz, E. Palle, D. Gandolfi et al., Mass determination of the 1:3:5 near-resonant planets transiting GJ 9827 (K2-135). Astron. Astrophys. 618, A116 (2018)

L. Puig, G. Pilbratt, A. Heske et al., The phase a study of the ESA M4 mission ARIEL. Exp. Astron. 46, 211 (2018)

S. Quanz et al., Atmospheric characterization of terrestrial exoplanets in the mid-infrared: biosignatures, habitability \& diversity (2019). Submitted to ESA in response to the Call for White Papers for the Voyage 2050 long-term plan. arXiv:1908.01316

A. Quirrenbach et al., CARMENES: an overview six months after first light. Proc. SPIE 990812, SPIE.9908 (2016)

J.M. Raines, G.A. DiBraccio, T.A. Cassidy, D. Delcourt, M. Fujimoto, X. Jia, V. Mangano, A. Milillo, M. Sarantos, J.A. Slavin, P. Wurz, Plasma sources in planetary magnetospheres: Mercury. Space Sci. Rev. (2015). https://doi.org/10.1007/s11214-015-0193-4

S. Rappaport, R. Sanchis-Ojeda, L.A. Rogers, A. Levine, J.N. Winn, The Roche limit for close-orbiting planets: minimum density, composition constraints, and application to the $4.2 \mathrm{hr}$ planet KOI 1843.03. Astrophys. J. 773, L15 (2013) 
H. Rauer, S. Gebauer, P. Von Paris, J. Cabrera, M. Godolt, J.L. Grenfell, A. Belu, F. Selsis, P. Hedelt, F. Schreier, Potential biosignatures in super-Earth atmospheres. I. Spectral appearance of super-Earths around M dwarfs. Astron. Astrophys. 529, A8 (2011). https://doi.org/10.1051/0004-6361/201014368

H. Rauer et al., The PLATO 2.0 mission. Exp. Astron. 38(1-2), 249-330 (2014)

F. Raulin, A. Coustenis, G. Kminek, N. Hedman, Special issue: planetary protection: new aspects of policy and requirements. Life Sci. Space Res. 23, 1-142 (2019). https://doi.org/10.1016/j.1ssr.2019.09.003

H. Rème, C. Aoustin, J.M. Bosqued, I. Dandouras et al., First multispacecraft ion measurements in and near the Earth's magnetosphere with the identical Cluster ion spectrometry (CIS) experiment. Ann. Geophys. 19, 1303-1354 (2001)

Report of the Venera-D Joint Science Definition Team, Venera-D: Expanding Our Horizon of Terrestrial Planet Climate and Geology Through the Comprehensive Exploration of Venus, January 31, 2019. http://www.iki.rssi.ru/events/2019/Venera-DPhaseIIFinalReport.pdf

I. Ribas, E.F. Guinan, M. Güdel, M. Audard, Evolution of the solar activity over time and effects on planetary atmospheres. I. High-energy irradiances (1-1700 ̊̊). Astrophys. J. (2005). https://doi.org/10.1086/427977

G.R. Ricker et al., Transiting Exoplanet Survey Satellite (TESS). J. Astron. Telesc. Instrum. Syst. 1, 014003 (2015)

A.R. Ridden-Harper, I.A.G. Snellen, C.U. Keller et al., Search for an exosphere in sodium and calcium in the transmission spectrum of exoplanet 55 Cancri e. Astron. Astrophys. 593, A129 (2016)

F. Rodler, Exoplanet Research in the Era of the Extremely Large Telescope (ELT). Handbook of Exoplanets (Springer, Berlin, 2018), p. 194. ISBN 978-3-319-55332-0

L. Rogers, Most 1.6 Earth radii planets are not rocky. Astrophys. J. 801, 41 (2015). https://doi.org/10.1088/ 0004-637X/801/1/41

D. Rouan, H.J. Deeg, O. Demangeon et al., The orbital phases and secondary transits of Kepler-10b. A physical interpretation based on the lava-ocean planet model. Astrophys. J. Lett. 741, L30 (2011)

S. Rugheimer, L. Kaltenegger, A. Zsom, A. Segura, D. Sasselov, Spectral fingerprints of Earth-like planets around FGK stars. Astrobiology 13, 251-269 (2013). https://doi.org/10.1089/ast.2012.0888

S. Rugheimer, L. Kaltenegger, A. Segura, J. Linsky, S. Mohanty, Effect of UV radiation on the spectral fingerprints of Earth-like planets orbiting M stars. Astrophys. J. 809 (2015). https://doi.org/ 10.1088/0004-637X/809/1/57

C. Sagan, Direct contact among galactic civilizations by relativistic interstellar spaceflight. Planet. Space Sci. 11, 485-498 (1963). https://doi.org/10.1016/0032-0633(63)90072-2

C. Sagan, W.R. Thompson, R. Carlson, D. Gurnett, C. Hord, A search for life on Earth from the Galileo spacecraft. Nature 365, 715-721 (1993)

Y. Saito et al., In-flight performance and initial results of plasma energy angle and composition experiment (PACE) on SELENE (Kaguya). Space Sci. Rev. (2010). https://doi.org/10.1007/s11214-010-9647-X

A.B. Sanin, I.G. Mitrofanov, Selection of Luna-25 and Luna-27 landing sites based on LRO data, in $A G U$ Fall General Assembly, vol. 2016, (2016), id. P53A-2182

A. Santerne, B. Brugger, D.J. Armstrong et al., An Earth-sized exoplanet with a Mercury-like composition. Nat. Astron. 2, 393 (2018)

T.E. Sarris, E.R. Talaat, M. Palmroth, I. Dandouras, E. Armandillo, G. Kervalishvili et al., Daedalus: a lowflying spacecraft for the exploration of the lower thermosphere - ionosphere. Geosci. Instrum. Method. Data Syst. (2020). https://doi.org/10.5194/gi-9-153-2020

J. Scalo, L. Kaltenegger, A. Segura, M. Fridlund, I. Ribas, Y.N. Kulikov, J.L. Grenfell, H. Rauer, P. Odert, M. Leitzinger, F. Selsis, M.L. Khodachenko, C. Eiroa, J.F. Kasting, H. Lammer, M stars as targets for terrestrial exoplanet searches and biosignature detection. Astrobiology 7, 85-166 (2007). https://doi.org/10.1089/ast.2006.0125

L. Schaefer, B. Fegley, Chemistry of silicate atmospheres of evaporating super-Earths. Astrophys. J. Lett. 703, L113 (2009)

M. Scherf, H. Lammer, N.V. Erkaev, K.E. Mandt, B. Marty, Nitrogen atmospheres of the icy bodies in the solar system. Space Sci. Rev. (2020). https://doi.org/10.1007/s11214-020-00752-0. in press

A. Schillings, R. Slapak, H. Nilsson, M. Yamauchi, I. Dandouras, L.-G. Westerberg, Earth atmospheric loss through the plasma mantle and its dependence on solar wind parameters. Earth Planets Space (2019). https://doi.org/10.1186/s40623-019-1048-0

E. Schlawin, T. Hirano, H. Kawahara et al., Back to "normal" for the disintegrating planet candidate KIC 12557548 b. Astron. J. 156, 28 (2018)

H. Schleicher, G. Wiedemann, H. Wöhl, T. Berkefeld, D. Soltau, Detection of neutral sodium above Mercury during the transit on 2003 May 7. Astron. Astrophys. 425, 1119 (2004)

E.W. Schwieterman, N.Y. Kiang, M.N. Parenteau, C.E. Harman, S. DasSarma, T.M. Fisher, G.N. Arney, H.E. Hartnett, C.T. Reinhard, S.L. Olson, V.S. Meadows, C.S. Cockell, S.I. Walker, J.L. Grenfell, S. Hegde, S. Rugheimer, R. Hu, T.W. Lyons, Exoplanet biosignatures: a review of remotely detectable signs of life. Astrobiology 18 (2017). https://doi.org/10.1089/ast.2017.1729 
S. Seager, The search for habitable planets with biosignature gases framed by a "biosignature drake equation.”. Int. J. Astrobiol. 17, 294-302 (2017). https://doi.org/10.1017/S1473550417000052

S. Seager, W. Bains, R. Hu, Biosignature gases in $\mathrm{H}_{2}$-dominated atmospheres on rocky exoplanets. Astrophys. J. 777, 95 (2013a). https://doi.org/10.1088/0004-637X/777/2/95

S. Seager, W. Bains, R. Hu, A biomass-based model to estimate the plausibility of exoplanet biosignature gases. Astrophys. J. 775, 104 (2013b). https://doi.org/10.1088/0004-637X/775/2/104

A. Segura, K. Krelove, J.F. Kasting, D. Sommerlatt, V. Meadows, D. Crisp, M. Cohen, E. Mlawer, Ozone concentrations and ultraviolet fluxes on Earth-like planets around other stars. Astrobiology 3, 689-708 (2003). https://doi.org/10.1089/153110703322736024

A. Segura, J.F. Kasting, V. Meadows, M. Cohen, J. Scalo, D. Crisp, R.A.H. Butler, G. Tinetti, Biosignatures from Earth-like planets around M dwarfs. Astrobiology 5, 706-725 (2005). https://doi.org/ 10.1089/ast.2005.5.706

K. Seki, M. Hirahara, T. Terasawa, T. Mukai, Y. Saito, S. Machida, T. Yamamoto, S. Kokubun, Statistical properties and possible supply mechanisms of tailward cold $\mathrm{O}^{+}$beams in the lobe/mantle regions. $\mathrm{J}$. Geophys. Res. (1998). https://doi.org/10.1029/97JA02137

D.G. Sibeck, R. Allen, H. Aryan, D. Bodewits et al., Imaging plasma density structures in the soft Xrays generated by SolarWind charge exchange with neutrals. Space Sci. Rev. (2018). https://doi.org/ 10.1007/s11214-018-0504-7

A. Silburt, E. Gaidos, Y. Wu, A statistical reconstruction of the planet population around Kepler solar-type stars. Astrophys. J. 799, 180 (2015). https://doi.org/10.1088/0004-637X/799/2/180

E. Sinukoff, A.W. Howard, E.A. Petigura et al., Mass constraints of the WASP-47 planetary system from radial velocities. Astron. J. 153, 70 (2017)

R. Slapak, A. Schillings, H. Nilsson, M. Yamauchi, L.-G. Westerberg, I. Dandouras, Atmospheric loss from the dayside open polar region and its dependence on geomagnetic activity: implications for atmospheric escape on evolutionary time scales. Ann. Geophys. (2017). https://doi.org/10.5194/angeo-35-721-2017

J.A. Slavin et al., MESSENGER observations of magnetic reconnection in Mercury's magnetosphere. Science (2009). https://doi.org/10.1126/science.1172011

J. Sohn, S. Oh, Y. Yi, Lunar cosmic ray radiation environments during Luna and Lunar Reconnaissance Orbiter missions. Adv. Space Res. (2014). https://doi.org/10.1016/j.asr.2014.05.011

S.C. Solomon et al., The MESSENGER mission to Mercury: scientific objectives and implementation. Planet. Space Sci. (2001). https://doi.org/10.1016/S0032-0633(01)00085-X

C. Sotin, G. Avice, J. Baker, A. Freeman, S. Madzunkov, T. Stevenson, N. Arora, M. Darrach, G. Lightsey, B. Marty, Cupid's arrow: a small satellite concept to measure noble gases in Venus' atmosphere, in 49th Lunar and Planetary Science Conference, 2018, The Woodlands, Texas. LPI Contribution, vol. 2083, (2018), id. 1763

T.J. Stubbs, R.R. Vondrak, W.M. Farrell, A dynamic fountain model for dust in the lunar exosphere, in Dust in Planetary Systems, ESA SP-643 (2007)

E.E. Stüeken, M.A. Kipp, M.C. Koehler, E.W. Schwieterman, B.W. Johnson, R. Buick, Modeling $\mathrm{pN}_{2}$ through geological time: implications for planetary climates and atmospheric biosignatures. Astrobiology 16, 949-963 (2016). https://doi.org/10.1089/ast.2016.1537

E.E. Stüeken, S.M. Som, M.W. Claire, S. Rugheimer, M. Scherf, L. Sproß, N. Tosi, Y. Ueno, H. Lammer, Mission to planet Earth: the first two billion years. Space Sci. Rev. 216, 41 (2020). https://doi.org/ 10.1007/s11214-020-00667-w

S. Sugita et al., The geomorphology, color, and thermal properties of Ryugu: implications for parent-body processes. Science (2019). https://doi.org/10.1126/science.aaw0422

P.W. Sullivan et al., The transiting exoplanet survey satellite: simulations of planet detections and astrophysical false positives. Astrophys. J. 809, 77 (2015)

F. Tabataba-Vakili, J.L. Grenfell, J.-M. Grießmeier, H. Rauer, Atmospheric effects of stellar cosmic rays on Earth-like exoplanets orbiting M-dwarfs. Astron Astrophys. 585 (2016). https://doi.org/10.1051/ 0004-6361/201425602

M. Takashi et al., Mineralogy and crystallography of some itokawa particles returned by the Hayabusa asteroidal sample return mission. Earth Planets Space 66, 82 (2014). https://doi.org/10.1186/ 1880-5981-66-82

P. Tamburo, A. Mandell, D. Deming, E. Garhart, Confirming variability in the secondary eclipse depth of the super-Earth 55 Cancri e. Astron. J. 155, 221 (2018)

K. Terada, S. Yokota, Y. Saito, N. Kitamura, K. Asamura, M.N. Nishino, Biogenic oxygen from Earth transported to the Moon by a wind of magnetospheric ions. Nat. Astron. (2017). https://doi.org/10.1038/ s41550-016-0026

N. Thomas, Detection of O III 5007-A forbidden line emission from the Io plasma torus. Astrophys. J. 414, L41 (1993) 
N. Thomas, High resolution spectra of Io's neutral potassium and oxygen clouds. Astron. Astrophys. 313, 306 (1996)

F. Tian, J.F. Kasting, K.J. Zahnle, Revisiting HCN formation in Earth's early atmosphere. Earth Planet. Sci. Lett. 308, 417-423 (2011). https://doi.org/10.1016/j.epsl.2011.06.011

G. Tinetti et al., A chemical survey of exoplanets with ARIEL. Exp. Astron. (2018). https://doi.org/ 10.1007/s10686-018-9598-X

G. Tinetti et al., The science of ARIEL, in Space Telescopes and Instrumentation 2016: Optical, Infrared, and Millimeter Wave. Proc. SPIE, vol. 9904 (2016). 99041X

W.A. Traub, Kepler exoplanets: a new method of population analysis (2016). arXiv:1605.02255

L. Tu, C.P. Johnstone, M. Güdel, H. Lammer, The extreme ultraviolet and X-ray Sun in time: highenergy evolutionary tracks of a solar-like star. Astron. Astrophys. 577, L3 (2015). https://doi.org/ 10.1051/0004-6361/201526146

O.J. Tucker, W.M. Farrell, R.M. Killen, D.M. Hurley, Solar wind implantation into the lunar regolith: Monte Carlo simulations of $\mathrm{H}$ retention in a surface with defects and the $\mathrm{H}_{2}$ exosphere. J. Geophys. Res. (2019). https://doi.org/10.1029/2018JE005805

D. Valencia, E. Paracha, A.P. Jackson, Can a machine learn the outcome of planetary collisions? Astrophys. J. (2019). https://doi.org/10.3847/1538-4357/ab2bfb

R.J. Vervack Jr., W.E. McClintock, R.M. Killen, A.L. Sprague, B.J. Anderson, M.H. Burger, E.T. Bradley, N. Mouawad, S.C. Solomon, N.R. Izenberg, Mercury's complex exosphere: results from MESSENGER's third flyby. Science (2010). https://doi.org/10.1126/science.1188572

A.A. Vidotto, H. Lichtenegger, L. Fossati et al., Characterization of the HD 219134 multi-planet system II. Stellar-wind sputtered exospheres in rocky planets b \& c. Mon. Not. R. Astron. Soc. 481, 5296 (2018)

M. Viso, Mars sample receiving facility or facilities? That is the question. Life Sci. Space Res. 23, 69-72 (2019). https://doi.org/10.1016/j.1ssr.2019.06.004

A. Vorburger, P. Wurz, S. Barabash, M. Wieser, Y. Futaana, M. Holmström, A. Bhardwaj, K. Asamura, Energetic neutral atom observations of magnetic anomalies on the lunar surface. J. Geophys. Res. 117, A07208 (2012). https://doi.org/10.1029/2012JA017553

A. Vorburger, P. Wurz, S. Barabash, M. Wieser, Y. Futaana, A. Bhardwaj, K. Asamura, Imaging the South Pole-Aitken basin in backscattered neutral hydrogen atoms. Planet. Space Sci. (2015). https://doi.org/ 10.1016/j.pss.2015.02.007

M. Voss, H.W. Bange, J.W. Dippner, J.J. Middelburg, J.P. Montoya, B. Ward, The marine nitrogen cycle: recent discoveries, uncertainties and the potential relevance of climate change. Philos. Trans. R. Soc. Lond. B, Biol. Sci. 368 (2013). https://doi.org/10.1098/rstb.2013.0121

J.C.G. Walker, P.B. Hays, J.F. Kasting, A negative feedback mechanism for the long-term stabilization of Earth's surface temperature. J. Geophys. Res. 86, 9776-9782 (1981). https://doi.org/10.1029/ JC086iC10p09776

S. Watanabe et al., Hayabusa2 arrives at the carbonaceous asteroid 162173 Ryugu-a spinning top-shaped rubble pile. Science 364, 268-272 (2019). https://doi.org/10.1126/science.aav8032

D.T. Welling, M. André, I. Dandouras, D. Delcourt, A. Fazakerley, D. Fontaine, J. Foster, R. Ilie, L. Kistler, J. Lee, M.W. Liemohn, J.A. Slavin, C.-P. Wang, M. Wiltberger, A. Yau, The Earth: plasma sources, losses, and transport processes. Space Sci. Rev. (2015). https://doi.org/10.1007/s11214-015-0187-2

E.T. Wolf, Assessing the habitability of the TRAPPIST-1 system using a 3D climate model. Astrophys. J. 839, L1 (2017). https://doi.org/10.3847/2041-8213/aa693a

G. Wurm, M. Trieloff, H. Rauer, Photophoretic separation of metals and silicates: the formation of Mercurylike planets and metal depletion in chondrites. Astrophys. J. 769, 78 (2013)

P. Wurz, H. Lammer, Monte-Carlo simulation of Mercury's exosphere. Icarus (2003). https://doi.org/ 10.1016/S0019-1035(03)00123-4

P. Wurz, U. Rohner, J.A. Whitby, C. Kolb, H. Lammer, P. Dobnikar, J.A. Martín-Fernández, The lunar exosphere: the sputtering contribution. Icarus (2007). https://doi.org/10.1016/j.icarus.2007.04.034

P. Wurz, J.A. Whitby, U. Rohner, J.A. Martín-Fernández, H. Lammer, C. Kolb, Self-consistent modelling of Mercury's exosphere by sputtering, micro-meteorite impact and photon-stimulated desorption. Planet. Space Sci. 58, 1599-1616 (2010)

Y. Xu, H.S. Fu, C. Norgren, S. Toledo-Redondo, C.M. Liu, X.C. Dong, Ionospheric cold ions detected by MMS behind dipolarization fronts. Geophys. Res. Lett. (2019). https://doi.org/10.1029/2019GL083885

M. Yamauchi, Terrestrial ion escape and relevant circulation in space. Ann. Geophys. (2019). https://doi.org/ 10.5194/angeo-37-1197-2019

M. Yamauchi, R. Slapak, Energy conversion through mass loading of escaping ionospheric ions for different Kp values. Ann. Geophys. (2018). https://doi.org/10.5194/angeo-36-1-2018

M. Yamauchi, J.-E. Wahlund, Role of the ionosphere for the atmospheric evolution of planets. Astrobiology 7, 783-800 (2007). https://doi.org/10.1089/ast.2007.0140 
A. Yau, M. André, Sources of ion outflow in the high latitude ionosphere. Space Sci. Rev. (1997). https://doi.org/10.1023/A:1004947203046

I. Yoshikawa, O. Korablev, S. Kameda, D. Rees, H. Nozawa, S. Okano, V. Gnedykh, V. Kottsov, K. Yoshioka, G. Murakami, F. Ezawa, G. Cremonese, The Mercury sodium atmospheric spectral imager for the MMO spacecraft of BepiColombo. Planet. Space Sci. 56, 224-237 (2009)

D.T. Young, J.L. Burch, R.G. Gomez, A. De Los Santos et al., Hot plasma composition analyzer for the magnetospheric multiscale mission. Space Sci. Rev. (2016). https://doi.org/10.1007/s11214-014-0119-6

K.J. Zahnle, Photochemistry of methane and the formation of hydrocyanic acid (HCN) in the Earth's early atmosphere. J. Geophys. Res. 91, 2819-2834 (1986). https://doi.org/10.1029/JD091iD02p02819

A.L. Zerkle, S. Mikhail, The geobiological nitrogen cycle: from microbes to the mantle. Geobiology 15, 343-352 (2017). https://doi.org/10.1111/gbi.12228

J.K. Zink, B.M.S. Hansen, Accounting for multiplicity in calculating eta Earth. Mon. Not. R. Astron. Soc. 487, 246-252 (2019). https://doi.org/10.1093/mnras/stz1246

S.S. Zumdahl, S.A. Zumdahl, Chemistry, 8th edn. (Cengage Learning, Belmont, 2010)

T.H. Zurbuchen et al., MESSENGER observations of the spatial distribution of planetary ions near Mercury. Science (2011). https://doi.org/10.1126/science.1211302 